BONN-TH-2009-06

0909.2025 [hep-th]

\title{
Computing Brane and Flux Superpotentials in F-theory Compactifications
}

\author{
Thomas W. Grimm, Tae-Won Ha, Albrecht Klemm and Denis Klevers 1 \\ Bethe Center for Theoretical Physics, Universität Bonn, \\ Nussallee 12, 53115 Bonn, Germany
}

\begin{abstract}
In four-dimensional F-theory compactifications with $\mathrm{N}=1$ supersymmetry the fields describing the dynamics of space-time filling 7-branes are part of the complex structure moduli space of the internal Calabi-Yau fourfold. We explicitly compute the flux superpotential in F-theory depending on all complex structure moduli, including the 7-brane deformations and the field corresponding to the axio-dilaton. Since fluxes on the 7-branes induce 5-brane charge, a local limit allows to effectively match the F-theory results to a D5-brane in a non-compact Calabi-Yau threefold with threeform fluxes. We analyze the classical and instanton contributions to the F-theory superpotential using mirror symmetry for Calabi-Yau fourfolds. The F-theory compactifications under consideration also admit heterotic dual descriptions and we discuss the identification of the moduli in this non-perturbative duality.
\end{abstract}

September 2009

\footnotetext{
${ }^{1}$ grimm, tha, aklemm, klevers@th.physik.uni-bonn.de
} 


\section{Contents}

1 Introduction

2 D7-brane superpotentials and mirror symmetry $\quad 6$

2.1 The flux superpotential for Calabi-Yau orientifolds . . . . . . . . . 7

2.2 The D7-brane superpotentials . . . . . . . . . . . . . . . . . 8

2.3 Mirror symmetry with branes in toric geometry . . . . . . . . . . . . . 9

2.3.1 Calabi-Yau manifolds as hypersurfaces ........... 10

2.3.2 Toric branes and their mirrors .............. 11

\begin{tabular}{ll|l|}
3 & Flux superpotentials in F-theory & 13 \\
\hline
\end{tabular}

3.1 Elliptic fourfolds and seven-branes in F-theory . . . . . . . . . . . . 14

3.2 The flux superpotential ....................... 15

3.3 Constructing elliptic fourfolds . . . . . . . . . . . . . . . 17

3.4 Heterotic/F-theory duality ................... 19

4 Elliptically fibered Calabi-Yau fourfold mirror pairs 21

4.1 The non-compact Calabi-Yau geometry with D-branes . . . . . . . . 22

4.2 The compact elliptic Calabi-Yau threefold . . . . . . . . . . . . . 23

4.3 Construction of the elliptically fibered Calabi-Yau fourfold . . . . . . . . 27

5 Mirror symmetry for Calabi-Yau fourfolds $\quad 33$

5.1 States and correlation function of the B-model . . . . . . . . . . 35

5.2 The Frobenius Algebras . . . . . . . . . . . . . . . . 38

5.3 Matching of the A-model and B-model Frobenius algebra . . . . . . . . 4 40

5.4 Application to elliptic fourfolds . . . . . . . . . . . . . 46 
6 Basics of enumerative geometry

6.1 Closed GW invariants ........................ 52

6.2 Open GW invariants . . . . . . . . . . . . . . . . . 54

\begin{tabular}{ll|l|}
7 & Conclusions & 55 \\
\hline
\end{tabular}

A Further topological data of the main example

\begin{tabular}{l|l|} 
B Further examples of fourfolds & 61 \\
\hline
\end{tabular}

B.1 Fourfold with $F_{0} \ldots \ldots \ldots \ldots \ldots$

B.2 Fourfold with $F_{1} \ldots \ldots \ldots \ldots \ldots$ 


\section{Introduction}

The study of four-dimensional string compactifications leading to effective supergravity theories with $\mathcal{N}=1$ supersymmetry is crucial to connect string theory with phenomenology. A prominent scenario yielding such minimally supersymmetric effective theories are Type II string compactifications with space-time filling D-branes [1, 2, 3, 4]. At the present stage it remains challenging to compute the characteristic functions encoding the four-dimensional physics explicitly without restricting to specific limits in space of compactification manifolds. In part this is due to the fact that the low amount of supersymmetry does not significantly restrict the form of most of the couplings in the effective theory. The situation improves, however, if one focuses on holomorphic couplings such as the $\mathcal{N}=1$ superpotential and gauge-coupling function.

The explicit computation of the $\mathcal{N}=1$ superpotential $W$ in a string compactification allows to infer some crucial information about the vacuum structure in the effective theory. In particular, in exploring the possible vacua of Type II string theory, the superpotential induced by non-trivial background fluxes has been studied intensively for various examples [2, 3]. This task has been tractable since in this case $W$ can be explicitly computed by solving the Picard-Fuchs system of differential equations which determine the moduli dependence of the holomorphic three-form on the Calabi-Yau manifold. The superpotential is then expressed in terms of period integrals of the internal Calabi-Yau threefold which encode the dependence on the closed string moduli, the complex structure moduli and the complex axio-dilaton. Much less explored is the dependence of the superpotential on the open and closed moduli in the presence of D-branes. The D-brane superpotential $W$ is generically induced by D5-brane charge [5], and hence can arise on D5-branes or on higher dimensional branes with gauge flux on their world-volume which induces D5-brane charge. Formally, the brane superpotential can be calculated by considering reductions of Witten's holomorphic Chern-Simons action [6]. However, its explicit computation is more involved and requires the study of the full open-closed moduli space.

A natural generalization to evaluate the open-closed superpotential would be to find an extended Picard-Fuchs system for the closed and open periods. Its solutions then encode the full superpotential in the vicinity of a D-brane. So far, this has not been achieved in generality, but only in specific D-brane settings [7, 8, 9, 10, 11, 12]. In [13, 14] it was proposed to use (non-compact) Calabi-Yau fourfolds to compute the D5-brane superpotential and a connection with F-theory was indicated. On the other hand, to compute D5-brane superpotentials, we have proposed in [15] a constructive method involving the blow-up of D5-brane curve in the Calabi-Yau threefold. A further alternative to the 
above methods is studied in [16].

In this work we will study the computation of the open-closed superpotential for space-time filling seven-branes with gauge flux on the internal part of their worldvolume. In order to do that we use the fact that seven-branes admit a natural geometrization in F-theory on an elliptically fibered Calabi-Yau fourfold. Here the seven-branes wrap fourcycles in the base of the elliptic fibration over which the fiber degenerates. Moreover, in Ftheory the seven-brane deformations, the complex structure deformations corresponding to closed Type IIB moduli, and the axio-dilaton are on the same footing. They all arise as complex structure deformations of the Calabi-Yau fourfold. The superpotential for these fields is the famous Gukov-Vafa-Witten superpotential induced by four-form fluxes [17]. Hence, their appearance in the superpotential is parameterized by the holomorphic four-form on the Calabi-Yau fourfold and the open-closed superpotential is obtained from the periods of this higher-dimensional Calabi-Yau geometry. In [18] this computation of the Type II open-closed superpotential has been carried out for the case of Type IIB orientifold compactifications on $K 3 \times T^{2} / \mathbb{Z}_{2}$ with D3/D7-branes via an embedding into F-theory on $K 3 \times K 3$.

In order to study the F-theory superpotential $W$ we use several powerful tools. We start with the construction of the mirror fourfolds which admit a small number of Kähler moduli and allow us to compute the classical terms in the superpotential by evaluating elementary topological data of the fourfold. The mirror elliptic fourfolds thus have a small number of complex structure moduli and we are able to compute the moduli dependence of the holomorphic four-form explicitly. To identify the properly normalized moduli directions, we also make use of the duality of F-theory and heterotic $E_{8} \times E_{8}$ string theory. It allows us to identify the dependence of $W$ on the closed string moduli as well as heterotic bundle moduli which map to deformations of 7-branes. Moreover, in the local limit the superpotential of a 7-brane with gauge flux should coincide with the superpotential of a D5-brane in local geometries. In the refs. [7, 8], the authors have computed the superpotential for D5-branes in non-compact geometries by evaluating the periods and the chain integrals directly. Mirror symmetry allows one to map the result back to the obstruction problem in the moduli space of special Lagrangians $L$ in Calabi-Yau threefolds where the superpotential in the large complex structure limit becomes a generating function of Gromov-Witten invariants for holomorphic disks ending on $L$. We use these invariants to identify the correct periods and we thereby determine the four-form flux element which corresponds to the 7-brane gauge flux. By doing so we effectively have embedded the deformation problem of a D5-brane into the complex structure deformation of a compact Calabi-Yau fourfold. 
This work is organized as follows. In section 2 we outline the general structure of the flux and brane superpotential in the context of mirror symmetry which we will exploit in our later computations. Then we provide the required technical tools of toric geometry to realize Calabi-Yau mirror pairs as well as D-branes. In section 3 we turn to the discussion of the F-theory fourfold compactifications and introduce the geometric realization of seven-branes as well as the form of the flux superpotential. Then we describe the construction of fourfold geometries that admit a particularly rich fibration structure. Furthermore, we present a very brief account of the non-perturbative heterotic/F-theory duality exploiting the spectral cover construction. Our focus there lies on the identification of F-theory moduli in terms of the moduli of the heterotic spectral cover and complex structure.

In section 4 we explicitly construct mirror pairs of elliptic Calabi-Yau fourfolds. Since we aim to compare local Calabi-Yau threefold setups with branes with the F-theory compactifications, we first discuss the local geometry and its brane content for the local Calabi-Yau threefold $\mathcal{O}(-3) \rightarrow \mathbb{P}^{2}$. Then we construct a compact threefold $\tilde{Y}_{3}$ containing the local threefold and finally obtain the fourfold by fibering $\tilde{Y}_{3}$ over a $\mathbb{P}^{1}$-base. Our whole analysis there will be guided by the F-theory interpretation of the constructed geometries, its seven-brane content and the comparison to the D-brane picture. We also discuss the heterotic/F-theory duality using the spectral cover construction in the context of the considered example. By doing so, we can explicitly identify the threefold $Y_{3}$, the mirror of $\tilde{Y}_{3}$, as the heterotic dual Calabi-Yau threefold and the open modulus as the bundle modulus.

We proceed with a general analysis of mirror symmetry on fourfolds and readily apply the introduced concepts to our main example in section 5. We show that the classical terms of the F-theory superpotential can be fixed by computing topological data of the Calabi-Yau fourfold. Crucially, to fix the normalizations of the classical terms, we perform a monodromy analysis for the fourfold conifold point by analytic continuation of the fourfold periods. As a byproduct, we discover a new conifold monodromy and show that the conifold periods admit an interesting leading behavior which is different from Calabi-Yau threefolds. In section 5 we also state our main results for the computation of the F-theory flux superpotential and its interpretation in terms of the flux and brane superpotential. We are able to deduce the form of the classical terms in the maximally logarithmic period at the large complex structure point. Then the computation of the flux superpotential relies on the identification of the threefold periods among the fourfold periods what is supported by the physical relevance of $Y_{3}$ for heterotic/F-theory duality. Furthermore, we single out the fourfold period that matches the open superpotential. Finally, we are able to deduce appropriate $G_{4}$-flux which induces a F-theory superpotential 
matching both the brane and flux superpotential.

We conclude with section [6 where we provide the basic background necessary to understand enumerative interpretation of the brane, flux and F-theory superpotentials. Furthermore, in appendix $\mathrm{A}$ we summarize more detailed geometrical data of the example fourfold studied in the main text. Finally, in appendix B we study two more fourfold geometries with more moduli.

\section{D7-brane superpotentials and mirror symmetry}

In recent years compactifications of Type IIB string theory yielding a four-dimensional effective theory with $\mathcal{N}=1$ supersymmetry have been studied intensively. One prominent setup are Calabi-Yau orientifold compactifications with space-time filling D7-branes and O7-planes [4, 2, 3]. Here the extended D-branes wrap four-cycles, i.e. divisors, in the internal compactification space. The orientifold geometry is obtained by dividing out a $\mathbb{Z}_{2}$ symmetry of a compact Calabi-Yau threefold $Z_{3}$.

In order to study the vacua of such $\mathcal{N}=1$ compactifications, it is crucial to determine the effective four-dimensional superpotential. We will focus on the part of the superpotential which is generated by three-from fluxes $F_{3}=\left\langle d C_{2}\right\rangle$ and $H_{3}=\left\langle d B_{2}\right\rangle$, where $C_{2}, B_{2}$ are the R-R and NS-NS two-forms, as well as two-form fluxes $F_{2}=\langle d A\rangle$ for the field strength of the $U(1)$ gauge-potential $A$ on the internal part of a D7-brane. The $U(1)$ flux $F_{2}$ is thus an element of $H^{2}(D, \mathbb{Z})$, where $D$ is the divisor in $Z_{3}$ wrapped by the D7-brane. The respective superpotentials are given by [17, 19, 20, 4]

$$
W_{\text {flux }}(z)=\int_{Z_{3}}\left(F_{3}-\tau H_{3}\right) \wedge \Omega_{3}, \quad W_{\mathrm{D} 7}(z, \zeta)=\int_{\mathcal{C}_{5}} F_{2} \wedge \Omega_{3},
$$

where $\tau=C_{0}+i e^{-\phi}$ is the axio-dilaton, and $\Omega_{3}$ is the holomorphic three-form on the Calabi-Yau manifold $Z_{3}$. Note that $W_{\text {flux }}$ only depends on the complex structure deformations of $Z_{3}$ due to the appearance of $\Omega_{3}$, while $W_{\mathrm{D} 7}$ will also depend on the deformation moduli $\zeta$ of the D7-brane. To see the latter, one notes that $\mathcal{C}_{5}$ is a five-chain which ends on the divisor $D$, i.e. one has $D \subset \partial \mathcal{C}_{5}$, and carries the information about the embedding of the D7-brane into $Z_{3}$. In the following we will discuss the two superpotentials (2.1) in more detail. 


\subsection{The flux superpotential for Calabi-Yau orientifolds}

Let us first discuss the flux superpotential $W_{\text {flux }}$. It can be evaluated in terms of the periods $\left(X^{A}, F_{A}\right)$ of the holomorphic three-form $\Omega_{3}$

$$
W_{\text {flux }}=\hat{N}_{A} X^{A}(z)-\hat{M}^{A} F_{A}(z), \quad X^{A}=\int_{A^{A}} \Omega_{3}, \quad F_{A}=\int_{B_{A}} \Omega_{3} .
$$

where $\left(\hat{M}_{A}, \hat{N}^{A}\right)=\left(M_{A}-\tau \tilde{M}_{A}, N^{A}-\tau \tilde{N}^{A}\right)$ are complex numbers with flux quantum numbers $\left(M_{A}, N^{A}\right)$ of $F_{3}$ and $\left(\tilde{M}_{A}, \tilde{N}^{A}\right)$ of $H_{3}$. We have also introduced a symplectic basis $\left(A^{A}, B_{A}\right)$ of three-cycles in $H_{3}\left(Z_{3}, \mathbb{Z}\right)$. The dependence of the $2 h^{2,1}\left(Z_{3}\right)+2$ periods $\left(X^{A}, F_{A}\right)$ on the $h^{2,1}\left(Z_{3}\right)$ complex structure moduli $z^{i}$ can be evaluated by solving a system of partial differential equations, the Picard-Fuchs equations $\mathcal{L}_{a}\left(X^{A}, F_{B}\right)=0$. Here $\mathcal{L}_{a}$ are linear differential operators in the complex structure moduli $z^{i}$, which can be determined as reviewed, for example, in [21].

It is important to point out that by special geometry the non-trivial information about the $F_{A}$ periods can be encoded by a single holomorphic function, the prepotential $F^{0}\left(X^{A}\right)$, which is homogeneous of degree two in the periods $X^{A}$, such that the $F_{A}$ can be written as $F_{A}=\partial F^{0} / \partial X^{A}$.

There are only a few general observations which can be made about the flux superpotential $W_{\text {flux }}$, since the form of $W_{\text {flux }}$ will highly depend on the point at which it is evaluated on the complex structure moduli space. One particularly interesting point is the large complex structure point which by mirror symmetry corresponds to a large volume compactification of Type IIA string theory. By the known monodromy of the NS-NS $B$-field shift in the Type IIA theory one knows that the point must be of maximal unipotent monodromy, which implies on the B-model side a maximal logarithmic degeneration of periods near this point $z_{i}=0$ [22, 21]. More specifically, one can identify $X^{0} \propto \mathcal{O}(z)$ as the fundamental period having no logarithmic dependence on $z^{i}$, while $X^{i} \propto \log z+\mathcal{O}(z), F_{i} \propto(\log z)^{2}+\mathcal{O}(z)$ and $F_{0} \propto(\log z)^{3}+\mathcal{O}(z)$ are always logarithmic in the $z^{i}$. Mirror symmetry maps the log-terms to classical large-volume contributions while the regular terms in the $F_{i}$ encode the closed string world-sheet instantons corrections. To see this one notes that the mirror map takes the form $z_{i}=e^{2 \pi i t_{i}}+\ldots$, where $t^{i}=X^{i} / X^{0}$ is the world-sheet volume complexified with the NS-NS B-field on the Type IIA side. The prepotential $F^{0}$ encodes the classical couplings as well as the genus zero world-sheet instantons and takes the general form

$$
F^{0}=-\frac{1}{3 !} \mathcal{K}_{i j k} t^{i} t^{j} t^{k}-\frac{1}{2 !} \mathcal{K}_{i j} t^{i} t^{j}+\mathcal{K}_{i} t^{i}+\frac{1}{2} \mathcal{K}_{0}+\sum_{\beta} n_{\beta}^{0} \operatorname{Li}_{3}\left(q^{\beta}\right)
$$


where $q^{\beta}=e^{2 \pi i \beta_{j} t^{j}}$ for a vector $\beta$ with entries $\mathbb{Z}_{\geq 0}$. Here

$$
\begin{array}{ll}
\mathcal{K}_{i j k}=\int_{\tilde{Z}_{3}} J_{i} \wedge J_{j} \wedge J_{k}, & \mathcal{K}_{i j}=\frac{1}{2} \int_{\tilde{Z}_{3}} \imath_{*}\left(c_{1}\left(J_{j}\right)\right) \wedge J_{i}, \\
\mathcal{K}_{j}=\frac{1}{2^{2} 3 !} \int_{\tilde{Z}_{3}} c_{2}\left(T_{\tilde{Z}_{3}}\right) \wedge J_{j}, & \mathcal{K}_{0}=\frac{\zeta(3)}{(2 \pi i)^{3}} \int_{\tilde{Z}_{3}} c_{3}\left(T_{\tilde{Z}_{3}}\right) .
\end{array}
$$

are determined by the classical intersections of the mirror Calabi-Yau threefold $\tilde{Z}_{3}$ of $Z_{3}$. Note that by $c_{1}\left(J_{j}\right)$ we mean the first Chern class of the divisor associated to $J_{j}$ and $\imath_{*}=P_{\tilde{Z}_{3}} i_{*} P_{J_{j}}^{-1}$ is the Gysin homomorphism where $P_{\tilde{Z}_{3}}\left(P_{\tilde{J}_{j}}\right)$ is the Poincaré-duality map on $\tilde{Y}\left(J_{j}\right)$ and $i_{*}$ is the push-forward on the homology. Thus, $\imath_{*}\left(c_{1}\left(J_{j}\right)\right)$ is a fourform. The constants $n_{m}^{0}$ are the integral Gopakuma-Vafa invariants (BPS numbers) which can be computed explicitly for a given example by solving the Picard-Fuchs differential equation. Inserting the form of the pre-potential (2.3) into the flux superpotential (2.2) with $\hat{M}^{0}=0$ one finds

$$
W_{\text {flux }}=\hat{N}_{0}+\hat{N}_{i} t^{i}-\hat{M}^{i}\left[\frac{1}{2} \mathcal{K}_{i j k} t^{j} t^{k}+\mathcal{K}_{i j} t^{j}+\mathcal{K}_{i}+\sum_{\beta} \beta_{i} n_{\beta}^{0} \operatorname{Li}_{2}\left(q^{\beta}\right)\right]
$$

The equation (2.5) means that in addition to a cubic classical polynomial, also instanton correction terms proportional to $\operatorname{Li}_{2}(q)=\sum_{k=1}^{\infty} \frac{z^{k}}{k^{2}}$ are induced by non-vanishing flux $M^{i}$.

\subsection{The D7-brane superpotentials}

Let us now turn to the superpotential for the D7-brane. Ideally one would like to compute the functional dependence of $W_{\mathrm{D} 7}$ on the D7-deformations $\zeta$ and complex structure moduli $z$ by also evaluating a set of open-closed Picard-Fuchs equations. As we will see in section 3 this can be indeed achieved if one lifts the setup to an F-theory compactification on a Calabi-Yau fourfold. One can, however, already infer some crucial property of $W_{\mathrm{D} 7}$ by applying mirror symmetry at the large complex structure/large volume point. Recall that under mirror symmetry, a Type IIB compactification with D7-branes is mapped to a Type IIA compactification with D6-branes. In a supersymmetric configuration these

D6-branes wrap special Lagrangian cycles $L$ in the mirror Calabi-Yau space $\tilde{Z}_{3}$. The dimension of $H^{1}(L, \mathbb{Z})$ gives precisely the number of classical deformations $\hat{t}$ of $L$. The superpotential on the mirror side is then induced by string world-sheet discs which end on $L$.

We summarize the enumerative geometry involved in the counting problem for discs ending on $L$ in more detail in section 6. Here, let us note that the superpotential induced by the open string world-sheets takes the form

$$
W_{\mathrm{D} 7}=C_{i} t^{i} \hat{t}+C_{i j} t^{i} t^{j}+C \hat{t}^{2}+\sum_{\beta, n} n_{\beta, n}^{0} \operatorname{Li}_{2}\left(q^{\beta} Q^{n}\right), \quad Q=e^{2 \pi i \hat{t}} .
$$


with constants $C, C_{i}, C_{i j}$ and $n_{\beta, n}^{0}$ determined by the D-brane and $Z_{3}$ geometry, as well as the flux $F_{2}$. One finds that the full D7 superpotential contains both classical terms as well as instanton corrections which again has the $\mathrm{Li}_{2}$ structure as in (2.5).

While the D7-superpotential (2.1), (2.6) has not been studied extensively its D5-brane analogue is studied in many works. Clearly, since both are induced by D5-brane charge, it is important to summarize some of the literature on the D5-brane superpotential. The most thoroughly studied cases are mirror geometries of non-compact toric Calabi-Yau manifolds with Harvey-Lawson type branes [7, 8]. In these examples the brane superpotential, i.e. the chain integral, is calculated directly using a meromorphic differential naturally given in the mirror geometry. For compact geometries, only recently there has been much progress in computing the superpotential. For D5-branes which are mirror to D6-branes wrapping a rigid involution special Lagrangian cycle, the superpotential can be calculated by deriving an inhomogeneous Picard-Fuchs system and solving it [10, 11]. Extending the works [9] to compact examples, a method to derive a Picard-Fuchs system for open/closed moduli space is proposed using an auxiliary hypersurface [12]. In [14] it is proposed to compute the superpotential for toric branes by extending the polyhedron describing the ambient toric variety to one dimension higher polyhedron. The authors of [14, 12] propose that the two last methods are equivalent. Unfortunately, at least up to now, neither of the two methods provides a constructive algorithm to tackle a given D5-brane configuration, since in both formalisms one effectively has to work on an auxiliary divisor and pick the correct linear combination for the D5-superpotential by hand. In [15] we have proposed another more constructive method to compute the superpotential for D5-brane. Concretely, we argued that the deformation problem for a D5-brane in a Calabi-Yau threefold with variable complex structure is equivalent to considering the complex structure of a non-Calabi-Yau threefold which is canonically obtained by blowing up the original Calabi-Yau manifold along the curve which the D5-brane wraps. Work in computing explicit examples is in progress.

\subsection{Mirror symmetry with branes in toric geometry}

After giving a general overview of the superpotential and its relation to mirror symmetry, we now discuss the toric realization of mirror symmetry and branes [23, 7, 8]. At this point we will also introduce notions and techniques of toric geometry that are inevitable for the rest of this work. 


\subsubsection{Calabi-Yau manifolds as hypersurfaces}

A powerful tool to construct Calabi-Yau manifolds $Y_{n}$ and their mirrors $\tilde{Y}_{n}$ for an arbitrary complex dimension $n$ is by realizing them as hypersurfaces in toric ambient spaces. These hypersurfaces are specified by reflexive polyhedra [24].

For threefolds we start with IIA on a Calabi-Yau $\tilde{Y}_{3}$ which is mirror dual to Type IIB on $Y_{3}$. We realize the compact Calabi-Yau $\tilde{Z}_{3}$ as the hypersurface in a toric ambient variety $\tilde{V}_{4}$ constructed from a pair of reflexive polyhedra $\Delta_{4}^{\tilde{Y}}$ and $\Delta_{4}^{Y}$ in lattices $N, M$, that are dual, i.e. $\Delta_{4}^{\tilde{Y}}=\left(\Delta_{4}^{Y}\right)^{*} 2$ In general, the dual polyhedron $\Delta^{*}$ of a given polyhedron $\Delta$ in a lattice $M$ is defined as the set of points $p$ in the real span $N_{\mathbb{R}}=N \otimes \mathbb{R}$ of the dual lattice $N$ of $M$ such that

$$
\Delta_{4}^{*}=\left\{p \in N_{\mathbb{R}} \mid\langle q, p\rangle \geq-1 \text { for all } q \in \Delta_{4}\right\}
$$

Let us assume that the combinatorics of $\tilde{V}_{4}$ associated to the polyhedron $\Delta_{4}^{\tilde{Y}}$ is encoded in $k$ charge vectors $\ell^{(j)}$ describing the relations among the $m=k+4$ vertices $\tilde{v}_{i}$. The Calabi-Yau $\tilde{Y}_{3}$ is then given as the hypersurface $\{\tilde{f}=0\}$ in $\tilde{V}_{4}$ where $\tilde{f}$ is given as the following polynomial [24]

$$
\tilde{f}=\sum_{q \in \Delta_{4}^{Y} \cap M} \tilde{a}_{q} \prod_{i} \tilde{x}_{i}^{\left\langle\tilde{v}_{i}, q\right\rangle+1}
$$

in the $m$ projective coordinates $\tilde{x}_{j}$ of $\tilde{V}$ associated to each vertex $\tilde{v}_{j}$. This formula provides a direct way to count the number of complex structure parameters $\tilde{a}_{q}$ (up to automorphisms of $\tilde{V}_{4}$ ) by counting the integral points $q \in \Delta_{4}^{Y}$. Furthermore, $\tilde{Y}_{3}$ is CalabiYau since (2.8) contains the monomial $\tilde{x}_{1} \ldots \tilde{x}_{m}$ corresponding to the origin in $\Delta_{4}^{Y}$ so that $f$ is a section of the anti-canonical bundle $K_{\tilde{V}_{4}}^{*}=\mathcal{O}\left(\sum_{i} D_{i}\right)$, where $D_{i}=\left\{\tilde{x}_{i}=0\right\}$ is a toric divisor.

For the case of hypersurfaces in toric varieties, (closed string) mirror symmetry [24] is realized in a very elegant way. The mirror threefold $Y_{3}$ on the Type IIB side is obtained by simply interchanging the roles of $\Delta_{4}^{\tilde{Y}}$ and $\Delta_{4}^{Y}$ so that (2.8) describes $Y_{3}$ as the hypersurface in the toric variety $V_{4}$ associated to the polyhedron $\Delta_{4}^{Y}$,

$$
f=\sum_{p \in \Delta_{4}^{\tilde{Y}} \cap N} a_{p} \prod_{i} x_{i}^{\left\langle v_{i}, p\right\rangle+1} .
$$

Here, we again associated the projective coordinates $x_{i}$ to each vertex $v_{i}$ of $\Delta_{4}^{Y}$. Indeed, the necessary requirements for mirror symmetry, $h^{1,1}\left(Y_{3}\right)=h^{2,1}\left(\tilde{Y}_{3}\right)$ and $h^{2,1}\left(Y_{3}\right)=$

\footnotetext{
${ }^{2}$ For convenience we denote quantities in Type IIA always with ' $\sim$ ' in order to omit them for their mirror quantities on the Type IIB side where we will mainly work.
} 
$h^{1,1}\left(\tilde{Y}_{3}\right)$, are fulfilled for this construction. This is obvious from Batyrev's formula for the Hodge numbers [24] of a given $n$-fold $Y_{n}$ in a toric ambient space specified by $\Delta_{n+1}^{Y}$

$$
\begin{aligned}
h^{n-1,1}\left(Y_{n}\right) & =h^{1,1}\left(\tilde{Y}_{n}\right) \\
& =l\left(\Delta_{n+1}^{\tilde{Y}}\right)-(n+2)-\sum_{\operatorname{dim} \tilde{\theta}=n} l^{\prime}(\tilde{\theta})+\sum_{\operatorname{codim} \tilde{\theta}_{i}=2} l^{\prime}\left(\tilde{\theta}_{i}\right) l^{\prime}\left(\theta_{i}\right), \\
h^{1,1}\left(Y_{n}\right) & =h^{n-1,1}\left(\tilde{Y}_{n}\right) \\
& =l\left(\Delta_{n+1}^{Y}\right)-(n+2)-\sum_{\operatorname{dim} \theta=n} l^{\prime}(\theta)+\sum_{\operatorname{codim} \theta_{i}=2} l^{\prime}\left(\theta_{i}\right) l^{\prime}\left(\tilde{\theta}_{i}\right) .
\end{aligned}
$$

In this expression $\theta(\tilde{\theta})$ denote faces of $\Delta_{4}^{Y}\left(\Delta_{4}^{\tilde{Y}}\right)$, while the sum is over pairs $\left(\theta_{i}, \tilde{\theta}_{i}\right)$ of dual faces. The $l(\theta)$ and $l^{\prime}(\theta)$ count the total number of integral points of a face $\theta$ and the number inside the face $\theta$, respectively. Finally, $l(\Delta)$ is the total number of integral points in the polyhedron $\Delta$. Using these formulas one notes that polyhedra with a small number of points will correspond to Calabi-Yau fourfolds with few Kähler moduli, i.e. small $h^{1,1}$, and many complex structure moduli $h^{3,1}$. Since $h^{1,1}$ and $h^{3,1}$ are exchanged by mirror symmetry Calabi-Yau fourfolds with small $h^{3,1}$ are obtained as mirror manifolds of hypersurfaces specified by a small number of lattice points in the polyhedron.

For the case of Calabi-Yau fourfolds $\left(\tilde{X}_{4}, X_{4}\right)$ the complete list of model dependent Hodge numbers is $h^{1,1}\left(X_{4}\right), h^{3,1}\left(X_{4}\right), h^{2,1}\left(X_{4}\right)$ and $h^{2,2}\left(X_{4}\right)$, However only three of these are independent due to the Hirzebruch-Riemann-Roch index theorem implying [25]

$$
h^{2,2}\left(X_{4}\right)=2\left(22+2 h^{1,1}\left(X_{4}\right)+2 h^{3,1}\left(X_{4}\right)-h^{2,1}\left(X_{4}\right)\right) .
$$

Therefore, only $h^{2,1}\left(X_{4}\right)$ has to be calculated in addition to fix the basic topological data of $\left(\tilde{X}_{4}, X_{4}\right)$. Analogously to (2.10) it is readily given by the symmetric expression

$$
h^{2,1}\left(X_{4}\right)=h^{2,1}\left(\tilde{X}_{4}\right)=\sum_{\operatorname{codim} \tilde{\theta}_{i}=3} l^{\prime}\left(\tilde{\theta}_{i}\right) l^{\prime}\left(\theta_{i}\right) .
$$

This finally enables us to calculate the Euler number of fourfolds by

$$
\chi\left(X_{4}\right)=\chi\left(\tilde{X}_{4}\right)=6\left(8+h^{3,1}+h^{1,1}-h^{2,1}\right) .
$$

\subsubsection{Toric branes and their mirrors}

To a setup of mirror pairs $\left(Z_{3}, \tilde{Z}_{3}\right)$ of toric Calabi-Yau hypersurfaces we want to add open string degrees of freedom which introduce the so-called Harvey-Lawson type branes. 
This type of branes have been studied intensively in local toric Calabi-Yau threefolds in refs. [7, 8]. Note that in these examples the toric variety itself is a non-compact CalabiYau threefold. Since we want to cover also the non-compact case we will work in the following with a toric space of dimension $m-k$ denoted by $\tilde{V}_{m-k}$, where $m-k=3$ in the non-compact case, and $m-k=4$ in the compact case as in section 2.3.1.

The toric variety $\tilde{V}_{m-k}$ is represented as a symplectic quotient $\mathbb{C}^{m} / / G$ defined first by imposing vanishing moment maps

$$
\sum_{j=1}^{m} \ell_{j}^{(i)}\left|\tilde{x}_{j}\right|^{2}=r^{i}
$$

and second by dividing by the isometry or gauge group $G=U(1)^{k}$ as $\tilde{x}_{j} \mapsto e^{i \ell_{j}^{(i)} \epsilon_{i}} x_{j}[26$. Then solving (2.15) and using coordinates $p_{j}=\left|\tilde{x}_{j}\right|^{2}, \theta_{j}$ the toric variety $\tilde{V}_{m-k}$ can be visualized as a $T^{m-k}$-fibration over a real $(m-k)$-dimensional base $\mathbb{B}_{m-k}$ [27, 7]. The degeneration loci of the $T^{m-k}$-fibration where one or more $S^{1}$ shrink are on the boundary of $\mathbb{B}_{m-k}$ which is determined by $p_{j}=0$ or intersections thereof since $p_{j} \geq 0$.

In Type IIA the Harvey-Lawson type branes wrap special Langrangian cycles $L$ which can be specified by $r$ additional brane charge vectors $\hat{\ell}^{(a)}$ restricting the $\left|x_{j}\right|^{2}$ and their angles $\theta_{i}$ in the toric ambient variety $\tilde{V}_{m-k}$ so that [7]

$$
\sum_{j=1}^{m} \hat{\ell}_{j}^{(a)}\left|\tilde{x}_{j}\right|^{2}=c^{a}, \quad \theta_{i}=\sum_{a=1}^{r} \hat{\ell}_{i}^{(a)} \phi_{a}
$$

for angular parameters $\phi_{a}$. To fulfill the 'special' condition of $L$, which is equivalent to $\sum_{i} \theta_{i}=0$, one demands $\sum_{j} \hat{\ell}_{j}^{(a)}=0$.

This construction was used in non-compact Calabi-Yau threefolds $\tilde{Z}_{3}=\mathbb{C}^{k+3} / / G$ for which the Calabi-Yau condition $\sum_{j} \ell_{j}^{(i)}=0$ has to hold [7, 8]. Then A-branes introduced by (2.16) are graphically represented as real codimension $r$ subspaces of the toric base $\mathbb{B}_{3}$. The case which was considered for the non-compact examples in [8] is $r=2$ where the non-compact three-cycle $L$ is represented by a straight line ending on a point when projected onto the base $\mathbb{B}_{3}$. The generic fiber is a $T^{2}$ so that the topology of $L$ is just $\mathbb{R} \times S^{1} \times S^{1}$. However, upon tuning the moduli $c^{a}$ it is most convenient to move the $L_{a}$ to the boundary of $\mathbb{B}_{3}$ where two $\left\{p_{j}=0\right\}$-planes intersect. Then one of the two moduli is frozen, and one $S^{1}$ pinches such that the topology becomes $\mathbb{C} \times S^{1}$. These D6-branes are mirror to non-compact D5-branes which intersect a Riemann surface at a point. Later on, we will use the D5-brane results of [7, 8] in order to study the superpotential (2.1) of D7-branes with gauge flux $F_{2}$ on compact Calabi-Yau manifolds. The gauge flux induces an effective D5-charge on the D7-brane and we will be able to compare the D5-brane 
superpotential of [7, 8] to the D7-brane superpotential with appropriate $F_{2}$ in the local limit.

The mirror Type IIB description [23, 7] can be obtained as follows. First the mirror Calabi-Yau $Y_{3}$ is determined by a polynomial $W$ and $n$ constraints given by

$$
W=\sum_{j=0}^{m} y_{j}, \quad \prod_{j=0}^{m} y_{j}^{\ell_{j}^{(i)}}=z^{i}, \quad i=1, \ldots, n
$$

where the $z^{i}$ denote the complex structure moduli of $Z_{3}$ that are related to the (complexified) Kähler moduli $t^{i}$ of $\tilde{Y}_{3}$ by $z^{i}=e^{2 \pi i t^{i}}$. We note that we introduced a further coordinate $y_{0}$ for which we also have to include a zeroth component $\ell_{0}^{(i)}=-\sum_{j=1}^{m} \ell_{j}^{(i)}$. For compact threefolds $Y_{3}$ is then obtained as the orbifolded hypersurface

$$
\left\{W\left(x_{i}\right)=0\right\} / \Gamma
$$

in the mirror toric variety $V_{4}$ with homogeneous coordinates $x_{i}$. These are introduced by a change of coordinates such that the constraints in (2.17) are automatically solved. The map from $y_{i}$ to $x_{i}$ as well as the orbifold group $\Gamma$ is determined by the etalé map which is worked out for weighted projective spaces in [21, 24]. In the non-compact case, $Z_{3}$ is similarly

$$
x z=W\left(x_{i}\right)
$$

in affine coordinates $x, z$ of $\mathbb{C}$ and $x_{i} \in \mathbb{C}^{*}$, respectively.

Analogously the B-branes on holomorphic submanifolds $\mathcal{C}$ in $Z_{3}$ are specified by

$$
\prod_{j=0}^{m} y_{j}^{\hat{\ell}_{j}^{(a)}}=\epsilon^{a} e^{-c^{a}}, \quad a=1, \ldots, r
$$

that can also be re-expressed in terms of the coordinates $x_{i}$. The phases $\epsilon^{a}$ are dual to the Wilson line background of the flat $\mathrm{U}(1)$-connection on the special Lagrangian $L$ and complexify the moduli $c^{a}$ to the open moduli $\zeta^{a}$ [28]. As is clear from (22.20) the B-brane is supported over a holomorphic cycle $\mathcal{C}$ of complex codimension $r$. Thus for the configuration $r=2$ the mirror is a D5-Brane. Other cases can be considered as well leading to mirrors given by D7-branes on divisors $(r=1)$ or D3-branes on points $(r=3)$.

\section{$3 \quad$ Flux superpotentials in F-theory}

F-theory provides a geometrization of $\mathcal{N}=1$ Type IIB backgrounds with holomorphically varying complexified string coupling constant $\tau$ [29]. The parameter $\tau$ is interpreted 
as the complex structure modulus of a two-torus which can be fibered over the spatial dimensions of the Type IIB target space that is a Kähler manifold with positive curvature. The $(3+1)$-complex-dimensional geometry obtained this way captures non-trivial monodromies of $\tau$ around degeneration loci of the two-torus. Precisely this provides a geometrization of (non-perturbative) seven-branes, which include D7-branes and O7branes as special cases. In this section we discuss compactifications of F-theory to four space-time dimensions with a focus on the induced flux superpotential [17] inherited from the M-theory description of F-theory [4].

\subsection{Elliptic fourfolds and seven-branes in F-theory}

Let us study the four-dimensional effective $\mathcal{N}=1$ theory which arises by compactification of F-theory on an elliptically fibered fourfold $X_{4} \rightarrow B_{3}^{X}$ over a complex three-dimensional base $B_{3}^{X}$. This corresponds to Type IIB string theory compactified on $B_{3}^{X}$ with an axiodilaton $\tau=C_{0}+i e^{-\phi}$ varying holomorphically over the Kähler base $B_{3}^{X}$, i.e. one has

$$
\text { F-theory on } X_{4}=\text { Type IIB on } B_{3}^{X} \text {. }
$$

The Weierstrass form of the elliptic fibration of $X_{4}$ is given by

$$
y^{2}=x^{3}+f(u) x z^{4}+g(u) z^{6}
$$

where $f(u)$ and $g(u)$ vary over the base $B_{3}^{X}$ with coordinates $u$. To ensure that (3.2) is well-defined, $(x, y, z)$ are sections of $\left(\mathcal{O}_{B}, \mathcal{O}_{B}, K_{B}\right)$ and $(f, g)$ are sections of $\left(K_{B}^{-4}, K_{B}^{-6}\right)$, where $K_{B}$ is the canonical bundle of the base $B_{3}^{X}$. Equation (3.2) with the defined scalings for the coordinates $(x, y, z)$ implies that the generic elliptic fiber is $\mathbb{P}^{2}(1,2,3)[6]$, i.e. a degree 6 hypersurface in weighted projective space $\mathbb{P}^{2}(1,2,3)$. This will be the case for all examples considered in this work even if $X_{4}$ becomes singular.

As the axio-dilaton of Type IIB string theory $\tau$ corresponds to the complex structure of the elliptic fiber, it can be specified by the value of the classical $S L(2, \mathbb{Z})$ modular invariant $j$-function which is expressed through the functions $f$ and $g$ in (3.2) as

$$
j(\tau)=\frac{4(24 f)^{3}}{\Delta}, \quad \Delta=27 g^{2}+4 f^{3} .
$$

The function $j(\tau)$ admits a large $\operatorname{Im} \tau$ expansion $j(\tau)=e^{-2 \pi i \tau}+744+\mathcal{O}\left(e^{2 \pi i \tau}\right)$ from which we can read off the monodromy of $\tau$ around a 7 -brane.

In general, the elliptic fibration will be singular over the discriminant $\Delta$. It can factorize into several components which individually correspond to divisors $D_{i}$ in $B_{3}^{X}$ which are wrapped by seven-branes including the well-known D7-branes and O7-planes. The 
singularities of the elliptic fibration over the $D_{i}$ determine the gauge group on the sevenbranes. These can be determined explicitly using generalizations of the Tate formalism [30]. The weak string coupling limit of F-theory is given by $\operatorname{Im} \tau \rightarrow \infty$ and yields a consistent orientifold setup with D7-branes on a Calabi-Yau manifold [31].

It is important to note that the degeneration of the elliptic fibration can be so severe that the Calabi-Yau fourfold $X_{4}$ as given in (3.2) becomes singular. In this case it is not possible to work with the singular space directly since the topological quantities such as the Euler characteristic and intersection numbers are not well-defined. To remedy this problem the singularities can be systematically blown up to obtain a smooth geometry [30. In the cases considered in this paper this is done using the methods of toric geometry [30, 32, 33]. The resulting smooth geometry still contains the information about the gauge-groups on the seven-branes and allows to analyze the compactification in detail.

In this work we will entirely focus on the complex structure sector of the CalabiYau fourfold $X_{4}$. We will consider smooth spaces $X_{4}$ which only admit a small number $h^{3,1}\left(X_{4}\right)$ of complex structure deformations, but are obtained from singular elliptically fibered Calabi-Yau fourfolds by multiple blow-ups. This affects only the number of Kähler moduli, which we will not discuss in the following. In order to compare to the Type IIB weak coupling picture the complex structure moduli can be split into three classes [4]:

(1) One complex modulus corresponding to the complex axio-dilaton $\tau$ parametrizing the complex structure of the elliptic fiber.

(2) The moduli corresponding to the deformations of the seven-branes wrapped on divisors on $B_{3}^{X}$.

(3) The complex structure moduli corresponding to the deformations of the basis and its double covering Calabi-Yau threefold obtained in the orientifold limit.

\subsection{The flux superpotential}

It is well-known that F-theory admits a superpotential upon switching on four-form flux $G_{4}$. For even second Chern class of $X_{4}$ this flux is integer quantized ${ }^{3} G_{4} \in H^{4}\left(X_{4}, \mathbb{Z}\right)$ 34. To determine the F-theory superpotential one uses the duality between M-theory and F-theory [4, 35]. In an M-theory compactification on $X_{4}$ one encounters the famous Gukov-Vafa-Witten superpotential

$$
W(z)=\int_{X_{4}} G_{4} \wedge \Omega,
$$

\footnotetext{
${ }^{3}$ To be precise [34] we note that $G_{4}$ is quantized such that $\left[G_{4}-c_{2}\left(X_{4}\right) / 2\right] \in H^{4}\left(X_{4}, \mathbb{Z}\right)$.
} 
where $\Omega$ is the holomorphic $(4,0)$ form on $X_{4}$. The superpotential $W(z)$ depends on the complex structure deformations $z$ of the fourfold $X_{4}$. As we will discuss momentarily, upon imposing restrictions on the allowed fluxes $G_{4}$, the superpotential (3.4) also provides the correct expression for an F-theory compactification. The goal of this work is to explicitly compute (3.4) for specific elliptically fibered Calabi-Yau fourfolds. The result is then matched with the superpotentials (2.1) at weak string coupling such that

$$
\int_{X_{4}} G_{4} \wedge \Omega \rightarrow \int_{Z_{3}}\left(F_{3}-\tau H_{3}\right) \wedge \Omega_{3}+\sum_{m} \int_{\mathcal{C}_{5}^{m}} F_{2}^{m} \wedge \Omega_{3},
$$

where $m$ labels all D7-branes on divisors $D_{m}$ carrying two-form fluxes $F_{2}^{m}$.

Note that already by a pure counting of the flux quanta encoded by $G_{4} \in H^{4}\left(Y_{4}, \mathbb{Z}\right)$, as well as $F_{3}, H_{3} \in H^{3}\left(Z_{3}, \mathbb{Z}\right)$ and $F_{2}^{m} \in H^{2}\left(D_{m}, \mathbb{Z}\right)$ one will generically encounter a mismatch. This can be traced back to the fact that not all fluxes $G_{4}$ are actually allowed in an F-theory compactification, since in the duality between M-theory on $X_{4}$ and Ftheory on $X_{4}$ one of the dimensions of the elliptic fiber will become to a space-time dimension [4]. The simplest case with only D7-branes with abelian gauge groups is discussed, for example, in ref. [4]. In this situation the flux $G_{4}$ is allowed to have only components satisfying

$$
\int G_{4} \wedge \omega_{1} \wedge \omega_{2}=0, \quad \forall \omega_{1}, \omega_{2} \in H^{1,1}\left(X_{4}\right) .
$$

Our examples are, however, significantly more complicated and admit D7-branes with rather large gauge groups. This is due to the fact that we need to analyze fourfolds $X_{4}$ with few complex structure moduli, which typically have order a thousand elements of $H^{1,1}\left(X_{4}\right)$. Many of the elements in $H^{1,1}\left(X_{4}\right)$ will correspond to blow-ups of a singular elliptic fibration and signal the presence of enhanced gauge groups. Therefore, it will be more practical to discuss the matching of the moduli dependence encoded by the periods of $\Omega$ on $X_{4}$.

In the next step we want to extract the moduli dependence of the F-theory superpotential. Here we are aiming to state some general features of the flux superpotential. More details on the moduli dependence of $W$ will be presented in section 5. As in the threefold case (2.2) the fourfold superpotential can be expressed through the periods of $\Omega$. However, in the fourfold case the variations of the $(4,0)$ form $\Omega$ do not span the full cohomology $H^{4}\left(X_{4}\right)$, but rather only a subspace $H_{H}^{4}\left(X_{4}\right)$, known as the primary horizontal subspace of $H^{4}\left(X_{4}\right)$ [37]. It takes the form

$$
H_{H}^{4}\left(X_{4}, \mathbb{C}\right)=H^{4,0} \oplus H^{3,1} \oplus H_{H}^{2,2} \oplus H^{1,3} \oplus H^{0,4},
$$

where $H_{H}^{2,2}$ consists of the elements in $H^{2,2}$ which can be obtained as second variation of $\Omega$ with respect to the complex structure on $X_{4}$. The numbers $h_{H}^{4-i, i}\left(X_{4}\right)$ denote the 
dimension of the respective cohomologies in (3.7). Note that the fact that not all $H^{4}\left(X_{4}\right)$ can be reached as variations of $\Omega$ is in contrast to the Calabi-Yau threefold case. In the threefold case one can simply define the periods of $\Omega_{3}$ as in (2.2) by introducing an integral homology basis of $H_{3}\left(Y_{3}, \mathbb{Z}\right)$. In the fourfold case, however, one has to introduce a basis $\gamma_{a}^{(i)}$ of $H_{4}^{H}\left(X_{4}, \mathbb{Z}\right)$, in order to define the periods

$$
\Pi^{(i) a}=\int_{\gamma_{a}^{(i)}} \Omega, \quad i=0, \ldots, 4,
$$

where $\gamma_{a}^{(i)}$ for $a=1, \ldots, h_{H}^{4}\left(X_{4}\right)$ denote a graded basis of $H_{4}^{H}\left(X_{4}\right)$ with grade $i=0, \ldots, 4$. Here we also introduced the dual basis $\hat{\gamma}_{a}^{(i)}$ of $H_{H}^{4}\left(X_{4}, \mathbb{Z}\right)$ with pairing

$$
\int_{\gamma_{a}^{(i)}} \hat{\gamma}_{b}^{(j)}=\delta^{i j} \delta_{a b}
$$

This cohomology basis satisfies

$$
\begin{aligned}
\int_{X_{4}} \hat{\gamma}_{a}^{(i)} \wedge \hat{\gamma}_{b}^{(4-i)} & =\eta_{a b}^{(i)} \\
\int_{X_{4}} \hat{\gamma}_{a}^{(i)} \wedge \hat{\gamma}_{b}^{(j)} & =0 \text { for } i+j>4 .
\end{aligned}
$$

In this basis we expand the holomorphic four-form $\Omega=\sum_{i} \Pi^{(i) a} \hat{\gamma}_{a}^{(i)}$. Analogously, the flux quantization condition of $G_{4}$ reads in this basis of integral cycles

$$
G_{4}=\sum_{i} N^{(i) a} \hat{\gamma}_{a}^{(i)}, \quad N^{(i) a}=\int_{\gamma_{a}^{(i)}} G_{4},
$$

where $N^{(i) a}$ are integral flux numbers. Then in terms of these definitions the flux superpotential (3.4) can be expanded as

$$
W=\sum_{i} N^{(i) a} \Pi^{(4-i) b} \eta_{a b}^{(i)} \equiv \sum_{i} N^{(i) a} \Pi_{a}^{(4-i)}
$$

with the moduli independent intersection matrix $\eta_{a b}^{(i)}$ defined in (3.10). Note that as in the threefold case a direct definition of the integral basis $\gamma_{a}^{(i)}$ is impossible. However, the existence of such a basis can be inferred by using mirror symmetry at the large complex structure point. In fact, as in section 2.1 the periods $\Pi^{(i) a}$ can be selected according to their leading logarithmic behavior at this point as we will discuss in more detail in section 5 .

\subsection{Constructing elliptic fourfolds}

In the following we will discuss the construction of elliptically fibered Calabi-Yau fourfolds for which we want to compute the F-theory superpotential (3.4). Our strategy is to find 
fourfold examples $X_{4}$ which admit a small number of complex structure moduli such that we can evaluate the Picard-Fuchs equations determining the holomorphic four-form $\Omega$. Candidate examples have already been considered in refs. [38, 25]. Moreover, we construct the fourfolds in such a way that they contain a local Calabi-Yau patch in which the effective D5-brane superpotential has been computed explicitly [7, 8].

The Calabi-Yau fourfolds studied in this paper will be obtained as mirror dual to a Calabi-Yau threefold fibration over $\mathbb{P}^{1}$. Denoting by $\tilde{Y}_{3}$ the Calabi-Yau threefold fiber we can write this as

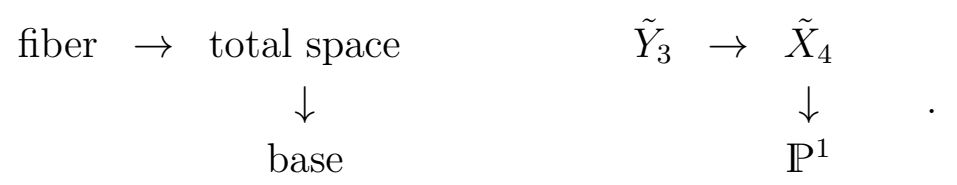

We will later pick Calabi-Yau threefolds $\tilde{Y}_{3}$ which are obtained by compactifications of local Calabi-Yau geometries which can support Harvey-Lawson type D6-branes as introduced in section 2.3. The compact Calabi-Yau threefolds $\tilde{Y}_{3}$ have small numbers $h^{1,1}$ of Kähler moduli, which is a feature inherited by $\tilde{X}_{4}$. Moreover, since we want to study F-theory on the mirror $X_{4}$ of $\tilde{X}_{4}$, the Calabi-Yau threefold $\tilde{Y}_{3}$ as well as the fibration structure of $\tilde{X}_{4}$ will be chosen carefully, such that $X_{4}$ has an elliptic fibration. This is achieved, for example, by choosing $Y_{3}$ elliptically fibered [44],

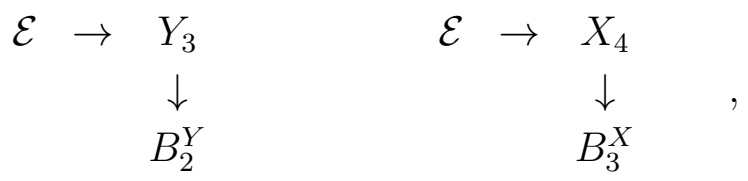

for which $X_{4}$ also admits a $K 3$-fibration with a heterotic dual on $Y_{3}$. Here $\mathcal{E}$ is the generic elliptic fiber and $B_{2}^{Y}$ and $B_{3}^{X}$ are the two-dimensional and three-dimensional base spaces of the fibrations, respectively. As we will show for the explicit examples, $\mathcal{E}=\mathbb{P}^{2}(1,2,3)[6]$ is the generic elliptic fiber shared by $Y_{3}$ and $X_{4}$.

To detect these fibration structures of a given mirror pair of Calabi-Yau fourfolds $\left(\tilde{X}_{4}, X_{4}\right)$ and in order to understand our construction more thoroughly it turns out to be sufficient to study the toric data in the corresponding reflexive polyhedra $\left(\Delta_{\tilde{X}_{4}}, \Delta_{X_{4}}\right)$ without computing the intersection numbers [39]. In fact, in our examples of spaces $X_{4}$ used in (3.1) the intersection numbers will be hard to compute because of their huge number of Kähler moduli. In the following we will recall the general theorem of ref. [39] and later, in section 4, apply it to our main example.

Suppose $\left(\tilde{X}_{4}, X_{4}\right)$ are given as hypersurfaces in the toric varieties constructed from the reflexive pair $\left(\Delta_{5}^{X}, \Delta_{5}^{\tilde{X}}\right)$ in the pair of dual lattices $(M, N)$. The statement of [39] gives two equivalent conditions for the existence of a Calabi-Yau fibration structure of 
the given fourfold $X_{4}$ once in terms of $\Delta_{5}^{X}$ and another time in terms of its dual $\Delta_{5}^{\tilde{X}}$. Assume there exists a $(n-k)$-dimensional lattice hyperplane in $N$ through the origin such that $\Delta_{k}^{F}:=H \cap \Delta_{5}^{X}$ is a $k$-dimensional reflexive polyhedron. Then this is equivalent with the existence of a projection $P$ to a $k$-dimensional sublattice of $M$ such that $P \Delta_{5}^{\tilde{X}}$ is a $k$-dimensional reflexive polyhedron $\Delta_{k}^{\tilde{F}}$ which is the dual of $\Delta_{k}^{F}$. If these conditions are satisfied, then the Calabi-Yau manifold $X_{4}$ which is obtained as a hypersurface of $\Delta_{5}^{X}$ has a Calabi-Yau fibration whose $(k-1)$-dimensional fiber $F_{k-1}$ is given by $\Delta_{k}^{F}$. The crucial point of these two equivalent criteria is that we can turn things around and analyze $X_{4}$ by not looking at hyperplanes $H$ in the complicated polyhedron $\Delta_{5}^{X}$, but at projections $P$ in $\Delta_{5}^{\tilde{X}}$ which is simple by construction. In both cases the base of the fibration can be found by considering the quotient polyhedron $\Delta_{5}^{X} / \Delta_{k}^{F}$ [33]. Here this quotient polyhedron is obtained by first determining the quotient lattice in $M \supset \Delta_{5}^{X}$ by dividing out the lattice generated by the integral points of $\Delta_{k}^{F}$. Then the integral points of $\Delta_{5}^{X} / \Delta_{k}^{F}$ are the equivalence classes of integral points in $\Delta_{5}^{X}$ in this quotient lattice. Schematically the analysis of the fibration structure can be summarized as

\begin{tabular}{|c|c|c|c|}
\hline Fibration structure & $\left(\Delta_{5}^{\tilde{X}}, \tilde{X}_{4}\right)$ & $\leftrightarrow$ & $\left(\Delta_{5}^{X}, X_{4}\right)$ \\
\hline $\begin{array}{c}\tilde{X}_{4} \text { admits } \\
\mathrm{CY}{ }_{m-1}-\text { fiber } \tilde{f}_{m-1}\end{array}$ & $\begin{array}{c}\text { Injection } \\
\Delta_{m}^{\tilde{f}}=\tilde{H} \cap \Delta_{5}^{\tilde{X}}\end{array}$ & $\leftrightarrow$ & $\begin{array}{c}\text { Projection } \\
\Delta_{m}^{f}=P \Delta_{5}^{X}\end{array}$ \\
\hline $\begin{array}{c}X_{4} \text { admits } \\
\mathrm{CY}_{k-1}-\text { fiber } F_{k-1}\end{array}$ & $\begin{array}{c}\text { Projection } \\
\Delta_{k}^{\tilde{F}}=P \Delta_{5}^{\tilde{X}}\end{array}$ & $\leftrightarrow$ & $\begin{array}{c}\text { Injection } \\
\Delta_{k}^{F}=H \cap \Delta_{5}^{X}\end{array}$ \\
\hline
\end{tabular}

where the arrow ' $\leftrightarrow$ ' indicates the action of mirror symmetry interchanging projection and injection. Clearly, this analysis can be also used to determine Calabi-Yau fibers $\tilde{f}_{m-1}$ of the mirror $\tilde{X}_{4}$. In general, it is not the case that mirror symmetry preserves fibration structures. However, in the constructions which we will analyze in the section 4, we will find that both $X_{4}$ and $\tilde{X}_{4}$ admit an intriguingly rich fibration structure

\subsection{Heterotic/F-theory duality}

In section 3.3 we have presented a construction of $X_{4}$ as the mirror of a fibration of the Calabi-Yau manifold $\tilde{Y}_{3}$ over $\mathbb{P}^{1}$. Specifically, we are interested in considering F-theory on the elliptic fourfold $X_{4}$. If $X_{4}$ also admits a K3 fibration, one can fiberwise apply the duality of F-theory on K3 with heterotic $E_{8} \times E_{8}$ strings on $T^{2}$ [29, 40, 42, 41]. For the four-dimensional compactification this implies that F-theory on $X_{4}$ is dual to the heterotic string on $Y_{3}$ [42, 43]. The gauge theory on the seven-branes arises either perturbatively from the ten-dimensional $E_{8} \times E_{8}$ or through non-perturbative effects in the heterotic 
string. In particular, the perturbative gauge group is encoded by two bundles $V_{1}$ and $V_{2}$ which encode the breaking of $E_{8} \times E_{8}$ to smaller gauge groups.

We are interested in the map of the complex structure moduli of $X_{4}$ to heterotic moduli. One first notes that the complex structure moduli of $Y_{3}$ are identified with complex structure moduli of $X_{4}$ under duality. In fact, one expects that the heterotic flux superpotential

$$
W_{\text {het }}=\int_{Y_{3}} H_{3} \wedge \Omega_{3},
$$

with $\mathrm{H}_{3}$ being the threeform flux background of the heterotic B-field, is mapped to a part of the F-theory flux superpotential. For the concrete examples considered in the rest of this paper we will show, that there indeed exist periods $\Pi_{a}^{(i)}$ of the holomorphic fourform $\Omega$ of $X_{4}$ which are identified with the threefold periods of $\Omega_{3}$ on $Y_{3}$. This provides an explicit map from the F-theory to the heterotic setup.

A second set of heterotic moduli which are mapped to the complex structure moduli of $X_{4}$ is a subset of the bundle moduli of $V_{1}, V_{2}$. To make this more precise one notes that one can specify certain non-trivial bundles on an elliptically fibered $Y_{3} \rightarrow B_{2}^{Y}$ by a spectral cover construction [45]. One first defines a flat bundle $\left.V\right|_{T^{2}}$ on the elliptic fiber $T^{2}$ and fibers these data over $B_{2}^{Y}$. Let us focus on $S U(N)$-bundles in the following. Such bundles can be specified by $N$ line bundles on $T^{2}$, or, in the dual picture, $N$ points on the dual torus [45]. Fibering these $N$ points over $B_{2}^{Y}$ one obtains a divisor in $Y_{3}$ that is an $N$-fold cover of $B_{2}^{Y}$, called the spectral cover. Concretely, for a Calabi-Yau threefold $Y_{3}$ of the Weierstrass form

$$
p_{0}=y^{2}+x^{3}+f x z^{4}+g z^{6}
$$

the data of the $N$ points on each elliptic fiber are specified by solutions to [45, 44]

$$
p_{+}=b_{0} z^{N}+b_{2} x z^{N-2}+b_{3} y z^{N-3}+\ldots+\left\{\begin{array}{l}
b_{N} x^{N / 2} \\
b_{N} y x^{(N-3) / 2}
\end{array},\right.
$$

where one distinguishes the cases $N$ even and $N$ odd. The $b_{i}$ are sections of a line bundle on $B_{2}^{Y}$. In general, they depend on moduli fields $\hat{z}_{i}$ which encode the deformations of the spectral cover and, hence, the bundle $V 4$

In the duality between the heterotic string on $\left(Y_{3}, V_{1}, V_{2}\right)$ and F-theory on $X_{4}$ the moduli $\hat{z}_{i}$ of the spectral cover are also mapped to complex structure moduli of $X_{4}$. This identification was made precise in ref. [45, 43, 47]. Roughly speaking, for an $S U(N) \times$ $S U(M)$ bundle on the heterotic side, the dual F-theory fourfold is given locally by the constraint of the form 44]

$$
\tilde{\mu}=p_{0}+v p_{+}+v^{-1} p_{-}=0
$$

\footnotetext{
${ }^{4}$ In general, there can be also Wilson line moduli. These are, however, absent for our examples.
} 
where $p_{0}=0$ specifies the threefold $Y_{3}$ as in (3.17), $p_{+}=0$ specifies an $S U(N)$ bundle $V_{1}$ as in (3.18), and $p_{-}$specifies an $S U(M)$ bundle $V_{2}$. The coordinate $v$ is the coordinate on the $\mathbb{P}^{1}$-basis in the $K 3$-fibers. For our concrete examples we will show that the open string moduli of the seven-branes in F-theory are precisely mapped to the coefficients of the spectral cover $p_{+}$. Switching on four-form fluxes $G_{4}$ in F-theory generates a superpotential for these fields which we will determine explicitly.

\section{Elliptically fibered Calabi-Yau fourfold mirror pairs}

The computation of the F-theory superpotential (3.4) will be done for a class of CalabiYau fourfolds $X_{4}$ that we will introduce here. Our strategy in constructing a fourfold $X_{4}$ with a low number of complex structure moduli is to construct its mirror $\tilde{X}_{4}$ as a Calabi-Yau threefold fibration $\tilde{Y}_{3}$ over a $\mathbb{P}^{1}$-base. The threefolds $\tilde{Y}_{3}$ we are interested in are themselves elliptically fibered and admit a local limit yielding the non-compact geometries $\mathcal{O}(K) \rightarrow B_{2}^{\tilde{Y}}$ studied in [8] where mirror symmetry with branes was analyzed in detail. This fact will be exploited when we analyze the seven-brane content of the F-theory setup $X_{4}$ and later on determine the F-theory flux superpotential which we split into flux and brane superpotential as in (3.5) .

Due to this chain of geometries it is natural to introduce the three geometries separately, where we will throughout our whole presentation focus on one concrete example for simplicity. We first summarize in section 4.1 the local geometry $\mathcal{O}(K) \rightarrow B_{2}^{\tilde{Y}}$ for the example of $B_{2}^{\tilde{Y}}=\mathbb{P}^{2}$ with D5-branes on its mirror geometry given by the Riemann surface $\Sigma$ of [8]. This non-compact geometry has both one complex structure modulus as well as one brane modulus of the D5-brane. Then we consider the threefold $\tilde{Y}_{3}$ obtained by compactifying this geometry in section 4.2. We will put emphasis on the singularities of the elliptic fibration of $Y_{3}$ and its interpretation when going back from $\tilde{Y}_{3}$ to the local geometry. One of the two complex structure moduli of $Y_{3}$ is fixed in the local limit whereas the second one appears as a parameter of one component of the discriminant of the elliptic fibration of $Y_{3}$. Finally in section 4.3 we will construct the fourfold $\tilde{X}_{4}$ and its mirror $X_{4}$ used for our four-dimensional F-theory compactification. There we will analyze the seven-brane content in detail. We will find that among the four complex structure moduli of $X_{4}$ one matches the complex structure of $\Sigma$ of the non-compact geometry and a second one introduces a brane modulus. Here the crucial point will be the geometrical interpretation of the appearance of this new modulus in F-theory opposed to the naive expectation from perturbative IIB with branes on $Y_{3}$. 


\subsection{The non-compact Calabi-Yau geometry with D-branes}

In the following we will discuss the local Calabi-Yau geometries in which the explicit computations of open and closed BPS numbers can be performed for the example of local $\mathbb{P}^{2}$, i.e. $\mathcal{O}(-3) \rightarrow \mathbb{P}^{2}$. Then we will consider the elliptically fibered Calabi-Yau threefold in the weighted projective space $\mathbb{P}^{4}(1,1,1,6,9)$ that contains the non-compact geometry in the limit of large elliptic fiber.

In [8] the non-compact $\mathcal{O}(-3) \rightarrow \mathbb{P}^{2}$ geometry with non-compact Harvey-Lawson branes was considered. The local Calabi-Yau is defined as the toric variety $\tilde{V}_{3}$ characterized by the polyhedron

$\left(\begin{array}{c|ccc|c|c} & \multicolumn{3}{|c|}{\Delta_{3}} & \ell^{(1)} & \\ \hline v_{1} & 0 & 0 & 1 & -3 & X_{0} \\ v_{1}^{b} & 1 & 1 & 1 & 1 & X_{1} \\ v_{2}^{b} & -1 & 0 & 1 & 1 & X_{2} \\ v_{3}^{b} & 0 & -1 & 1 & 1 & X_{3}\end{array}\right)$

where the superscript ${ }^{b}$ denotes the two-dimensional basis $\mathrm{P}^{2}$ and the $X_{i}$ denote homogeneous coordinates. The D-term constraint for this geometry reads

$$
-3\left|X_{0}\right|^{2}+\left|X_{1}\right|^{2}+\left|X_{2}\right|^{2}+\left|X_{3}\right|^{2}=0
$$

and $\tilde{V}_{3}$ can be viewed as a $\left(S^{1}\right)^{3}$-fibration over a three-dimensional base $\mathbb{B}_{3}$. The degeneration loci of the fiber, $\left|X_{i}\right|=0$, are shown in figure 1, The brane is defined torically by the brane charge vectors

$$
\hat{\ell}^{(1)}=(1,0,-1,0), \quad \hat{\ell}^{(2)}=(1,0,0,-1) .
$$

This leads to the two constraints

$$
\left|X_{0}\right|^{2}-\left|X_{2}\right|^{2}=c^{1}, \quad\left|X_{0}\right|^{2}-\left|X_{3}\right|^{2}=c^{2},
$$

where the $c^{a}$ denote the open string moduli. The brane geometry is $\mathbb{C} \times S^{1}$ and can be described by a one dimensional half line in the three real dimensional toric base geometry $\mathbb{B}_{3}$ ending on a line where two of the three $\mathbb{C}^{*}$-fibers degenerate. The A-brane has two inequivalent brane phases I and II as indicated in Figure 1.5

Mirror Symmetry for this geometry was analyzed in [8] where the disk instantons of the A-model were calculated exploiting the fact that the mirror geometry of $\mathcal{O}(-3) \rightarrow \mathbb{P}^{2}$ effectively reduces to the Riemann surface $\Sigma$ defined by $W\left(x_{1}, x_{2}\right)=0$ of (2.19)$)$. The

\footnotetext{
${ }^{5}$ Note that our phase II is precisely phase III of [8]. The phase II of $[8$, has been omitted since it is equivalent to phase I by symmetry of $\mathbb{P}^{2}$.
} 


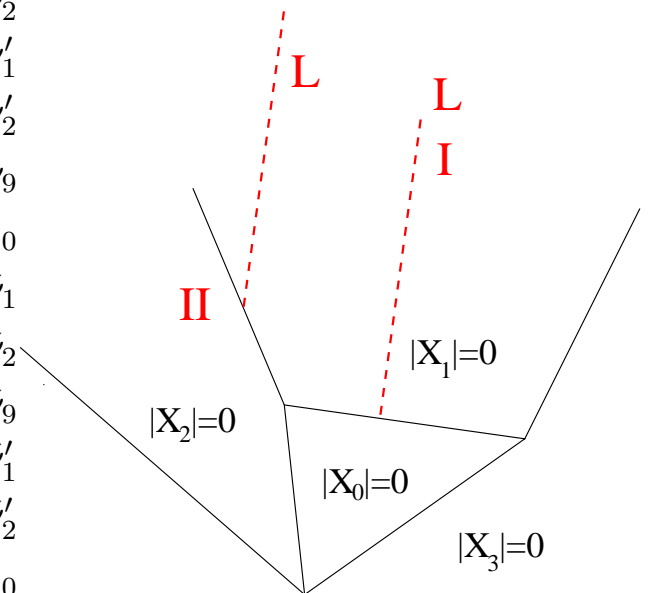

Figure 1: Toric base and Harvey-Lawson Lagrangians for non-compact $\mathbb{P}^{2}$

D6-brane is mapped under mirror symmetry to a D5-brane which intersects $\Sigma$ in a point. It will be this D5-brane picture which can be reformulated as a seven-brane with flux and embedded into an F-theory compactification.

\subsection{The compact elliptic Calabi-Yau threefold}

This local Calabi-Yau can easily be embedded into a compact Calabi-Yau threefold. The compactification can be understood as a replacement of the non-compact $\mathbb{C}$-fiber corresponding to $v_{1}$ of $\mathcal{O}(-3) \rightarrow \mathrm{P}^{2}$ by an elliptic fiber. Here we choose the generic fiber to be the elliptic curve in $\mathbb{P}^{2}(1,2,3)$ which we fiber over the $\mathbb{P}^{2}$-basis the same way as the non-compact $\mathbb{C}$-fiber before. Thus, the polyhedron of this compact threefold $\tilde{Y}_{3}$, its charge vectors, the homogeneous coordinates $\tilde{x}_{i}$ as well as the corresponding monomials for the mirror geometry $Y_{3}$, cf. (4.7), are given by

$$
\left(\begin{array}{c|cccc|cc|c|c} 
& \multicolumn{3}{|c|}{\Delta_{4}^{\tilde{Y}}} & & \ell^{(1)} & \ell^{(2)} & & \\
\hline v_{0} & 0 & 0 & 0 & 0 & 0 & -6 & \tilde{x}_{0} & z x y u_{1} u_{2} u_{3} \\
v_{1} & 0 & 0 & 2 & 3 & -3 & 1 & \tilde{x}_{1} & z^{6} u_{1}^{6} u_{2}^{6} u_{3}^{6} \\
v_{1}^{b} & 1 & 1 & 2 & 3 & 1 & 0 & \tilde{x}_{2} & z^{6} u_{3}^{18} \\
v_{2}^{b} & -1 & 0 & 2 & 3 & 1 & 0 & \tilde{x}_{3} & z^{6} u_{1}^{18} \\
v_{3}^{b} & 0 & -1 & 2 & 3 & 1 & 0 & \tilde{x}_{4} & z^{6} u_{2}^{18} \\
v_{2} & 0 & 0 & -1 & 0 & 0 & 2 & \tilde{x}_{5} & x^{3} \\
v_{3} & 0 & 0 & 0 & -1 & 0 & 3 & \tilde{x}_{6} & y^{2}
\end{array}\right) .
$$

Here the points $v_{1}, v_{2}, v_{3}$ carry the information of the elliptic fiber where we added the inner point $v_{1}$ in order to recover the $\mathbb{P}^{2}(1,2,3)$, in particular its homogeneous coordinate $\tilde{x}_{1}$ with weight one under the new $\mathbb{C}^{*}$-action $\ell^{(2)}$. Furthermore, applying the insights of 
(3.15), the elliptic fibration structure of $\tilde{Y}_{3}$ is obvious from the fact, that the polyhedron of $\mathbb{P}^{2}(1,2,3)$ occurs in the hyperplane $H=\{(0,0, a, b)\}$, but also as a projection $P$ on the (3-4)-plane indicating an elliptic fibration of the mirror $Y_{3}$, too 6

The polyhedron (4.5) corresponds to the degree 18 hypersurface in the weighted projective space $\mathbb{P}^{4}(1,1,1,6,9)$ blown up along the singular curve $\tilde{x}_{2}=\tilde{x}_{3}=\tilde{x}_{4}=0$ with exceptional divisor $v_{1}$. Its Euler number is $\chi=-540$ whereas $h^{1,1}=2, h^{2,1}=272$. Denoting the toric divisors $\tilde{x}_{i}=0$ by $D_{i}$, the two Kähler classes $J_{1}=D_{2}$ and $J_{2}=3 D_{2}+D_{1}$ correspond to the Mori vectors $\ell^{(1)}$ and $\ell^{(2)}$ in (4.5). They represent a curve in the hyperplane class of the $\mathbb{P}^{2}$ base and a curve in the elliptic fiber, respectively. The intersections of the dual divisors and the second Chern class are respectively computed to be 7

$$
\begin{aligned}
& \mathcal{C}_{0}=9 J_{2}^{3}+3 J_{2}^{2} J_{1}+J_{2} J_{1}^{2}, \\
& \mathcal{C}_{2}=102 J_{2}+36 J_{1} .
\end{aligned}
$$

In this notation the coefficients of the top intersection ring $\mathcal{C}_{0}$ are the cubic intersection numbers $J_{i} \cap J_{j} \cap J_{k}$, while the coefficients of $\mathcal{C}_{2}$ are $\left[c_{2}\left(T_{\tilde{X}}\right)\right] \cap J_{i}$.

Mirror symmetry for this example has been studied in [21, 48]. In order to construct the mirror pair $\left(Y_{3}, \tilde{Y}_{3}\right)$ as well as their constraints (2.8), (2.9) we need the dual polyhedron

$$
\left(\begin{array}{c|cccc|c} 
& \multicolumn{5}{|c|}{\Delta_{4}^{Y}} \\
\hline v_{1} & 0 & 0 & 1 & 1 & z \\
v_{1}^{b} & -12 & 6 & 1 & 1 & u_{1} \\
v_{2}^{b} & 6 & -12 & 1 & 1 & u_{2} \\
v_{3}^{b} & 6 & 6 & 1 & 1 & u_{3} \\
v_{2} & 0 & 0 & -2 & 1 & x \\
v_{3} & 0 & 0 & 1 & -1 & y
\end{array}\right)
$$

where again the basis was indicated by a superscript ${ }^{b}$. Again we added the inner point $v_{1}$ to recover the polyhedron of $\mathbb{P}^{2}(1,2,3)$ as the injection with $H=\{0,0, a, b\}$, thus confirming the elliptic fibration of the mirror $Y_{3}$. Here we distinguish between the twodimensional basis $B_{2}^{Y}=\mathbb{P}^{2}$ and the elliptic fiber by denoting the homogeneous coordinates of $\mathbb{P}^{2}(1,2,3)$ by $(z, x, y)$ and of $B_{2}^{Y}$ by $\left(u_{1}, u_{2}, u_{3}\right)$. The elliptic fibration structure reflects in particular in the constraint of $Y_{3}$ which takes a Weierstrass form

$$
p_{0}=a_{6} y^{2}+a_{5} x^{3}+a_{0} z x y u_{1} u_{2} u_{3}+z^{6}\left(a_{3} u_{1}^{18}+a_{4} u_{2}^{18}+a_{1} u_{1}^{6} u_{2}^{6} u_{3}^{6}+a_{2} u_{3}^{18}\right)=0 \text {. }
$$

\footnotetext{
${ }^{6}$ Besides the above chosen $(2,3)$, which leads to an elliptic fibration with one section, the values $(1,2)$ and $(1,1)$ are also admissible in the sense that these choices lead to reflexive polyhedra. The corresponding elliptic fibration has two and three sections, respectively.

${ }^{7}$ In performing these toric computations we have used the Maple package Schubert.
} 
The generic fiber can be seen by setting the coordinates $u_{i}$ of the basis $B_{2}^{Y}$ to some reference point, such that $p_{0}$ takes the form of a degree six hypersurface in $\mathbb{P}^{2}(1,2,3)$. The basis itself is obtained as the section $z=0$ of the elliptic fibration over $B_{2}^{Y}$.

The complex structure dependence of $Y_{3}$ is evident from the dependence of $p_{0}$ on the parameters $a_{i}$ which are coordinates on $\mathbb{P}^{6}$. However, they redundantly parameterize the complex structure of $Y$ due to the symmetries of $\mathbb{P}^{4}(1,1,1,6,9)$. Indeed there is a $\left(\mathbb{C}^{*}\right)^{6} /\left(\mathbb{C}^{*}\right)^{2}$ rescaling symmetry of the coordinates that enables us to eliminate four of the $a_{i}$ recovering the two complex structure parameters that match $h^{1,1}\left(\tilde{Y}_{3}\right)=h^{2,1}\left(Y_{3}\right)=2$. The appropriate coordinates $z_{i}$ obeying $z_{i}=0$ at the large complex structure/large volume point are completely determined by the phase of the A-model, i.e. the choice of charge vectors $\ell^{(i)}$ of $\Delta_{4}^{\tilde{Y}}$. They are given in general by

$$
z_{i}=(-1)^{\ell_{0}^{(a)}} \prod_{j=0}^{m} a_{j}^{\ell_{j}^{(i)}}
$$

which we readily apply for the situation at hand to obtain

$$
z_{1}=\frac{a_{2} a_{3} a_{4}}{a_{1}^{3}}, \quad z_{2}=\frac{a_{1} a_{5}^{2} a_{6}^{3}}{a_{0}}
$$

Thus, we can use the $\left(\mathbb{C}^{*}\right)^{4}$ action and the overall scaling to set $a_{i}=1, i=2, \ldots, 6$ for five parameters to obtain

$$
p_{0}=y^{2}+x^{3}+z x y m_{1}+z^{6} m_{6}
$$

where we have abbreviated

$$
m_{1}=z_{2}^{-1 / 6} z_{1}^{-1 / 18} u_{1} u_{2} u_{3}, \quad m_{6}=u_{1}^{18}+u_{2}^{18}+u_{3}^{18}+z_{1}^{-1 / 3} u_{1}^{6} u_{2}^{6} u_{3}^{6}
$$

Alternatively, this result can be obtained more directly by the mirror construction (2.17). In this case one needs the following assignment of coordinates $y_{i}$ to points of $\Delta_{4}^{\tilde{Y}}$ and monomials

$$
\left(\begin{array}{c|c|c}
y_{0} & v_{0} & a_{0} z x y u_{1} u_{2} u_{3} \\
y_{1} & v_{1} & a_{1} z^{6} u_{1}^{6} u_{2}^{6} u_{3}^{6} \\
y_{2} & v_{1}^{b} & a_{2} z^{6} u_{3}^{18} \\
y_{3} & v_{2}^{b} & a_{3} z^{6} u_{1}^{18} \\
y_{4} & v_{3}^{b} & a_{4} z^{6} u_{2}^{18} \\
y_{5} & v_{2} & a_{5} x^{3} \\
y_{6} & v_{3} & a_{6} y^{2}
\end{array}\right) .
$$

This defines the etalé-map that solves the constraints of (2.17) automatically when (4.10) holds. By setting $a_{0}=z_{2}^{-1 / 6} z_{1}^{-1 / 18}, a_{1}=z_{1}^{-1 / 3}$ and $a_{i}=1, i=2, \ldots, 6$ we solve (4.10) and $W=\sum_{j} y_{j}$ immediately reproduces $p_{0}$ in (4.11). 
Next we show that (4.11) indeed gives back the local geometry which is a conic over a genus one Riemann surface. The local limit in the A-model geometry is given by making the elliptic fiber infinitely large. This corresponds to $z_{2} \rightarrow 0$ in the B-model geometry. We parameterize $z_{2}$ by $\varepsilon \equiv z_{2}$ such that the local limit is given by $\varepsilon \rightarrow 0$. At the end we should obtain an affine equation, thus, using the two $\mathbb{C}^{*}$-action we set the coordinates $z$ and $u_{3}$ to one. By redefining the coordinates $x$ and $y$ as follows

$$
y \rightarrow \varepsilon^{-1 / 2} y+k_{1}^{1 / 2} \quad, \quad x \rightarrow \varepsilon^{-1 / 3} x+k_{2}^{2 / 3},
$$

the hypersurface equation $p_{0}=0$ becomes

$$
p_{0}=\frac{1}{\varepsilon} \tilde{p}_{0}+k_{1}^{2}+k_{2}^{2}+m_{6}=0
$$

where we set $z=1$ and $u_{3}=1$. Now we split the above equation

$$
\tilde{p}_{0}=\varepsilon \quad, \quad k_{1}^{2}+k_{2}^{2}+m_{6}=-1 .
$$

If we now take the $\varepsilon \rightarrow 0$ limit we obtain after appropriately redefining the $k_{i}$ the equation for the local geometry of the form

$$
u v=H(x, y)=x+1-\phi \frac{x^{3}}{y}+y .
$$

The Riemann surface defined by $H(x, y)=0$ is isomorphic to the surface $m_{6}=0$ up to isogeny.

As discussed in section 3.4 considering heterotic string theory on the elliptically fibered Calabi-Yau threefold $Y_{3}$ is expected to be dual to F-theory on $X_{4}$ if the fourfold admits a K3 fibration and its mirror is constructed as in (3.13) [44]. We have shown that $Y_{3}$ is indeed an elliptic fibration, and will confirm in the next section that $X_{4}$ is a K3 fibration. However, it is crucial to point out that there will be a large non-perturbative gauge group from the blown-up singularities of the elliptic fibration of $Y_{3}$. Upon introducing the full set of coordinates, i.e. introducing the inner points in $\Delta_{4}^{Y}$, one notes that the elliptic fibration not only degenerates over the curves $m_{6}=0$ and $432 m_{6}+m_{1}^{6}=0$ in the base of $Y_{3}$, but also over many curves described by the additional coordinates. Even though the determination of the non-perturbative gauge group will be not of importance in our analysis, let us point out that we will similarly find a large gauge group in the F-theory compactification on $X_{4}$. However, the identification of the moduli of the gauge bundles with the complex structure moduli of $X_{4}$ can still be performed by extracting the spectral cover constraint (3.19).

Before continuing with the construction of the Calabi-Yau fourfold, let us close with another comment on the use of the vectors $\hat{\ell}^{(1)}$ and $\hat{\ell}^{(2)}$ given in (4.3). On the compact 
threefold they translate to

$$
\hat{\ell}^{(1)}=(0,1,0,-1,0,0,0), \quad \hat{\ell}^{(2)}=(0,1,0,0,-1,0,0),
$$

due to the new origin in the polyhedron (4.5). In fact, applying (2.20) and using (4.13), they define the divisors

$$
z_{1}^{-1 / 3} u_{1}^{6} u_{2}^{6} u_{3}^{6}=\hat{z}_{1} u_{1}^{18}, \quad z_{1}^{-1 / 3} u_{1}^{6} u_{2}^{6} u_{3}^{6}=\hat{z}_{2} u_{2}^{18}
$$

in the compact $Y_{3}$. Here we introduced the moduli $\hat{z}_{a}$ corresponding to the charge vector $\hat{\ell}^{(a)}$. Note that in our F-theory compactification of the next section we will not consider seven-branes naively wrapped on these divisors as one would in a compactification of Type IIB on Calabi-Yau orientifolds. Rather we will construct a Calabi-Yau fourfold with 7-branes on its discriminant which possess additional moduli. These additional fields correspond to either $\hat{z}_{1}$ or $\hat{z}_{2}$ and allow deformations of the seven-brane constraint by the additional terms (4.19). Hence, $\hat{z}_{i}$ can be interpreted as deformations of the seven-brane divisors in $X_{4}$, or as spectral cover moduli in the heterotic dual.

\subsection{Construction of the elliptically fibered Calabi-Yau fourfold}

Having discussed the threefold geometry, we are now in the position to construct and analyze the elliptically fibered Calabi-Yau fourfold $X_{4}$ which is used in the F-theory compactification. Again, we start by constructing the mirror $\tilde{X}_{4}$ first. It is obtained by fibering the Calabi-Yau threefold $\tilde{Y}_{3}$ over a $\mathbb{P}^{1}$ such that one of the D-brane vectors $\hat{\ell}^{(i)}$ of the local model (4.1) appears as a new charge vector. As we will see later on, this new charge vector dictates the location of the moving seven-brane, while the second vector not used in the construction of the fourfold controls the volume of the $\mathbb{P}^{1}$-basis of the dual fourfold $\tilde{X}_{4}$ in (3.13). We will discuss the flux in more detail later on, but first proceed with the geometric construction. In the following we will exemplify our construction for a main example in detail, and we list the toric and geometrical data necessary to reproduce our results. Further examples are relegated to appendix B.

The Calabi-Yau fourfolds $\left(X_{4}, \tilde{X}_{4}\right)$ are realized as hypersurfaces in a toric ambient space described by a dual pair of reflexive polyhedra $\left(\Delta_{5}^{X}, \Delta_{5}^{\tilde{X}}\right)$. The reflexive polyhedron

$\Delta_{5}^{\tilde{X}}$ for a fibration of the toric variety constructed from $\Delta_{4}^{\tilde{Y}}$ over $\mathrm{P}^{1}$ is specified as follows

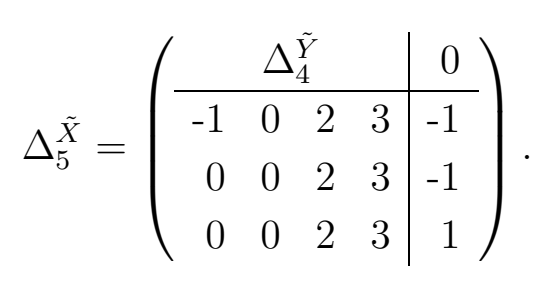


By construction, one finds $\Delta_{4}^{\tilde{Y}}$ by intersecting the hyperplane $\tilde{H}=\left(p_{1}, p_{2}, p_{3}, p_{4}, 0\right)$ with $\Delta_{5}^{\tilde{X}}$. Following $(3.15)$ this indeed identifies $\tilde{X}_{4}$ as a $\tilde{Y}_{3}$-fibration, and by performing the quotient $\Delta_{5}^{\tilde{X}} / \Delta_{4}^{\tilde{Y}}$ the base is readily shown to be the toric variety $((-1),(1))$, i.e. a $\mathbb{P}^{1}$. It is crucial to note that the additional points which do not lie on $\tilde{H}$ are constrained by two important conditions. Firstly, they are chosen of a form such that the mirror $X_{4}$ is elliptically fibered. This means, that using the projection to the third and fourth coordinate one finds the polyhedron of a torus in $\mathbb{P}^{2}(1,2,3)$ just as in the threefold case in section 4.1. The fact that $\tilde{X}_{4}$ is also elliptically fibered is not crucial in the construction. In particular, a similar construction can also be performed for the quintic hypersurface fibered over a $\mathbb{P}^{1}$, since the mirror quintic admits an elliptic fibration with generic elliptic fiber being a torus in $\mathrm{P}^{2}$. Secondly, the remaining entries are inserted such that one charge vector for the Calabi-Yau fourfold is of the form $\left(\hat{\ell}^{(1)},-,-,-\right)$. Adding this vector to form a higher-dimensional non-reflexive polyhedron was already proposed in [13, 14], 8

In the following we will choose to realize the open string vector $\hat{\ell}^{(1)}$ as in (4.20) to construct the $\mathbb{P}^{1}$-fibration 9 The Calabi-Yau fourfold $\tilde{X}_{4}$ is realized in the toric space described by the polyhedron $\Delta_{5}^{\tilde{X}}$. Its topological numbers are computed to be

$$
h^{3,1}=2796, \quad h^{1,1}=4, \quad h^{2,1}=0, \quad h^{2,2}=11244, \quad \chi=16848 .
$$

Here we first used (2.10), (2.11) as well as (2.13) and next applied (2.12), (2.14).

Note that $\Delta_{5}^{\tilde{X}}$ has three triangulations, which correspond to non-singular Calabi-Yau phases which are connected by flop transitions. In the following we will consider two of these phases in detail. These phases will match the two brane phases in figure 1 in the local Calabi-Yau threefold geometry.

To summarize the topological data of the Calabi-Yau fourfold for the two phases of

\footnotetext{
${ }^{8}$ Note that the interpretation of the construction in terms of the B-model in [13, 14] seems different from the F-theory interpretation given here.

${ }^{9}$ We could have used also $\hat{\ell}^{(2)}$, reproducing the same local D5-brane limit. In this case $\hat{\ell}^{(1)}$ specifies the gauge flux inducing the D5-brane charge on the D7-brane.
} 
interest, we specify the generators of the Mori cone $l_{I}^{(i)}$ and $l_{I I}^{(i)}$ for $i=1, \ldots 4$.

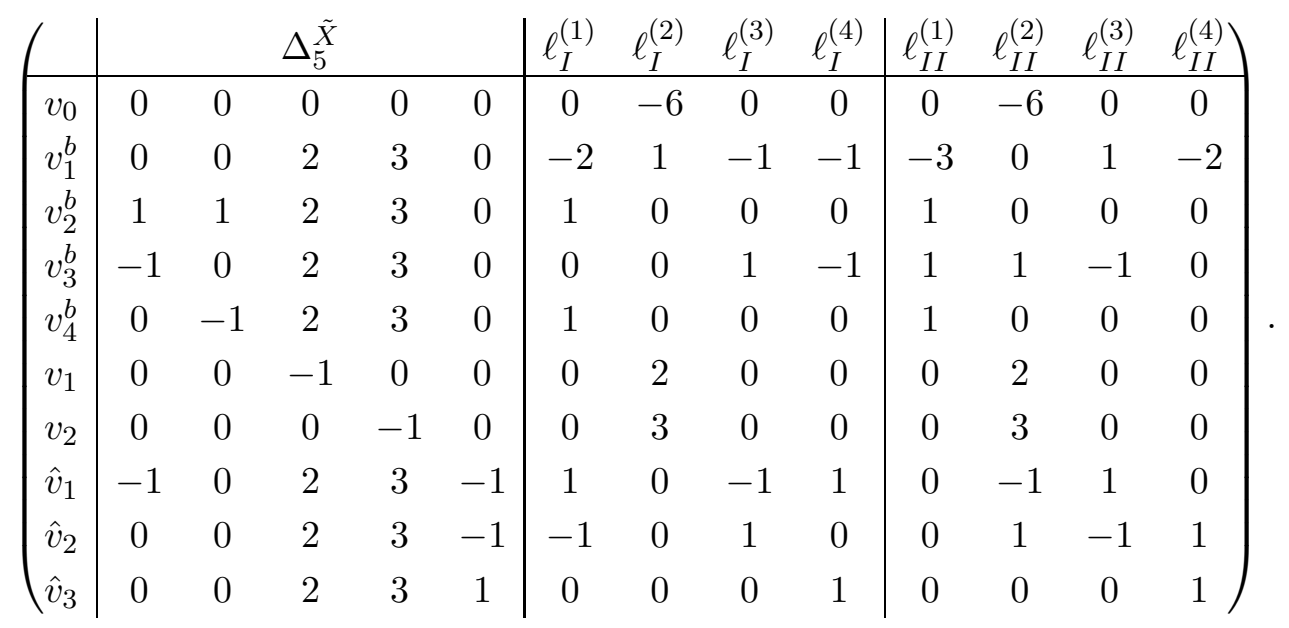

The charge vectors are best identified in the phase II. Here $\ell_{I I}^{(1)}$ and $\ell_{I I}^{(2)}$ are the extensions of the threefold charge vectors $\ell^{(1)}, \ell^{(2)}$ in $(4.5)$ to the fourfold. The brane vector $\hat{\ell}^{(1)}$ is visible in phases II as a subvector of $\ell_{I I}^{(3)}$. The remaining vector $\ell_{I I}^{(4)}$ arises since one had to complete the polyhedron such that it becomes reflexive implying that $\tilde{X}_{4}$ is a Calabi-Yau manifold. It represents the curve of the $\mathbb{P}^{1}$-basis of $\tilde{X}_{4}$. Phase I is related to phase II by a flop transition of the curve associated to $\ell_{I}^{(3)}$. Hence, in phase I the brane vector is identified with $-\ell_{I}^{(3)}$. Furthermore, after the flop transition one has to take

$$
\ell_{I I}^{(3)}=-\ell_{I}^{(3)}, \quad \ell_{I I}^{(1)}=\ell_{I}^{(1)}+\ell_{I}^{(3)}, \quad \ell_{I I}^{(2)}=\ell_{I}^{(2)}+\ell_{I}^{(3)}, \quad \ell_{I I}^{(4)}=\ell_{I}^{(4)}+\ell_{I}^{(3)}
$$

Note that the $\ell_{I}^{(i)}$ and $\ell_{I I}^{(i)}$ are chosen in such a way, that they parameterize the Mori cone of $\tilde{X}_{4}$. The dual Kähler cone generators for phase I are then given by

$$
J_{1}=D_{2}, \quad J_{2}=D_{1}+2 D_{2}+D_{3}+2 D_{9}, \quad J_{3}=D_{3}+D_{9}, \quad J_{4}=D_{9},
$$

where $D_{i}:=\left\{x_{i}=0\right\}$ are the nine toric divisors associated to the points $\Delta_{5}^{\tilde{X}}$ which differ from the origin. In phase II one has

$$
J_{1}=D_{2}, \quad J_{2}=D_{1}+2 D_{2}+D_{3}+2 D_{9}, \quad J_{3}=D_{1}+3 D_{2}+2 D_{9}, \quad J_{4}=D_{9} .
$$

The $J_{i}$ provide a distinguished integral basis of $H^{1,1}\left(\tilde{X}_{4}\right)$ since in the expansion of the Kähler form $J$ in terms of the $J_{i}$ all coefficients will be positive when parameterizing physical volumes of cycles in $\tilde{X}_{4}$. The $J_{i}$ are also canonically used as a basis in which one determines the topological data of $\tilde{X}_{4}$. The complete set of topological data of $\tilde{X}_{4}$ including the intersection ring as well as the non-trivial Chern classes are summarized in appendix A.

The polyhedron $\Delta_{5}^{\tilde{X}}$ has only few Kähler classes which makes it possible to identify part of the fibration structures from the intersection numbers. However, an analogous 
analysis is not possible for the mirror manifold $X_{4}$ since the dual polyhedron $\Delta_{5}^{X}$ has 2796 Kähler classes. Therefore, we apply the methods reviewed in section 3.3 in analyzing both $\tilde{X}_{4}$ and $X_{4}$. As already mentioned above, $\Delta_{5}^{\tilde{X}}$ intersected with the two hyperplanes

$$
H_{1}=\left(0,0, p_{3}, p_{4}, 0\right), \quad H_{2}=\left(p_{1}, p_{2}, p_{3}, p_{4}, 0\right) \text {. }
$$

gives two reflexive polyhedra corresponding to the generic torus fiber and the generic three-dimensional Calabi-Yau fiber $\tilde{Y}_{3}$. The fibration structures of the mirror CalabiYau $X_{4}$ is studied by identifying appropriate projections to $\Delta_{\tilde{F}}^{k} \subset \Delta_{5}^{\tilde{X}}$. Three relevant projections $P_{i}$ are

$$
P_{1}(\underline{p})=\left(p_{3}, p_{4}\right), \quad P_{2}(\underline{p})=\left(p_{1}, p_{2}, p_{3}, p_{4}\right), \quad P_{3}(\underline{p})=\left(p_{3}, p_{4}, p_{5}\right),
$$

where $\underline{p}=\left(p_{1}, \ldots, p_{5}\right)$ are the columns in the polyhedron $\Delta_{5}^{\tilde{X}}$. Invoking the theorem of section 3.3 , we see from $P_{1}$ that $X_{4}$ is also elliptically fibered and since the polyhedron of $\mathbb{P}^{2}(1,2,3)$ is self-dual, the fibration is of $\mathbb{P}^{2}(1,2,3)$ type. In addition, it is clear from $P_{2}$ that $X_{4}$ is Calabi-Yau threefold fibered. The fiber threefold is $Y_{3}$, the mirror to $\tilde{Y}_{3}$. The fact, that the threefold fibers of $X_{4}$ and $\tilde{X}_{4}$ are mirror manifolds is special to this example since the subpolyhedra obtained by $H_{2}$ and $P_{2}$ are identical. Finally, note that $X_{4}$ is K3 fibered as inferred from the projection $P_{3}$. This ensures the existence of a heterotic dual theory by fiberwise applying the duality of F-theory on K3 to heterotic strings on $T^{2}$. Replacing the K3-fiber by an elliptic fiber we find the Calabi-Yau threefold $Y_{3}$.

The hypersurface constraint for $X_{4}$ depends on the four complex structure moduli $z_{i}$. This dependence is already captured by only introducing 12 out of the many coordinates needed to specify a non-singular $X_{4}$. This subset of points in $\Delta_{5}^{X}$ is given by

$$
\Delta_{5}^{X} \supset\left(\begin{array}{r|rrrrr|r}
v_{1} & 0 & 0 & 1 & 1 & 0 & z \\
v_{2} & -12 & 6 & 1 & 1 & 0 & u_{1} \\
v_{3} & 6 & -12 & 1 & 1 & 0 & u_{2} \\
v_{4} & 6 & 6 & 1 & 1 & 0 & u_{3} \\
v_{5} & 0 & 0 & -2 & 1 & 0 & x \\
v_{6} & 0 & 0 & 1 & -1 & 0 & y \\
v_{1}^{b} & -12 & 6 & 1 & 1 & -6 & x_{1} \\
v_{2}^{b} & -12 & 6 & 1 & 1 & 6 & x_{2} \\
v_{3}^{b} & 6 & -12 & 1 & 1 & -6 & x_{3} \\
v_{4}^{b} & 6 & 6 & 1 & 1 & -6 & x_{4} \\
v_{5}^{b} & 0 & -6 & 1 & 1 & 6 & x_{5} \\
v_{6}^{b} & 0 & 6 & 1 & 1 & 6 & x_{6}
\end{array}\right)
$$

where we have omitted the origin. Note that we have displayed in (4.28) the vertices of $\Delta_{5}^{X}$ and added the inner points $v_{1}$ and $v_{2}$ to list all points necessary to identify the polyhedron $\Delta_{4}^{Y}$ with vertices (4.7) in the hyperplane orthogonal to $(0,0,0,0,1)$ and 
thus the Calabi-Yau fibration with fiber $Y_{3}$. The base of this fibration is given by the points labeled by a superscript ${ }^{b}$. Note that $(0,0,1,1,0)$ is also needed to observe the elliptic fibration. The base of the elliptic fibration is obtained by performing the quotient $\Delta_{3}^{\text {base }}=\Delta_{5}^{X} /\left(P_{1} \Delta_{5}^{\tilde{X}}\right)^{*}$ which amounts to simply dropping the third and fourth entry in the points of $\Delta_{5}^{X}$. Additionally, one can also see the elliptic fibration directly on the defining polynomial $\tilde{\mu}$ of $X_{4}$ which can be written in a Weierstrass form. Indeed if we apply (2.9) for the points displayed in (4.28) of $\Delta_{5}^{X}$ and all points $p$ of $\Delta_{5}^{\tilde{X}}$ that are not on codimension one faces we obtain a hypersurface of the form 10

$$
\tilde{\mu}=a_{6} y^{2}+a_{5} x^{3}+\tilde{m}_{1}\left(x_{j}, u_{i}\right) x y z+\tilde{m}_{6}\left(x_{j}, u_{i}\right) z^{6}=0 .
$$

Here $x_{j}, u_{i}$ are the homogeneous coordinates on the base of the elliptic fibration, while $x$, $y$, and $z$ are the homogeneous coordinates of the $\mathbb{P}^{2}(1,2,3)$ fiber. The polynomials $\tilde{m}_{1}$ and $\tilde{m}_{6}$ are given by

$$
\begin{aligned}
\tilde{m}_{1}\left(x_{j}, u_{i}\right)= & a_{0} u_{1} u_{2} u_{3} x_{1} x_{2} x_{3} x_{4} x_{5} x_{6}, \\
\tilde{m}_{6}\left(x_{j}, u_{i}\right)= & u_{1}^{18}\left(a_{7} x_{1}^{24} x_{2}^{12} x_{3}^{6} x_{4}^{6}+a_{3} x_{1}^{18} x_{2}^{18} x_{5}^{6} x_{6}^{6}\right)+a_{4} u_{2}^{18} x_{3}^{18} x_{5}^{12}+a_{2} u_{3}^{18} x_{4}^{18} x_{6}^{12} \\
& +u_{1}^{6} u_{2}^{6} u_{3}^{6}\left(a_{1} x_{1}^{6} x_{2}^{6} x_{3}^{6} x_{4}^{6} x_{5}^{6} x_{6}^{6}+a_{9} x_{2}^{12} x_{5}^{12} x_{6}^{12}+a_{8} x_{1}^{12} x_{3}^{12} x_{4}^{12}\right),
\end{aligned}
$$

where the $a_{i}$ denote coefficients encoding the complex structure deformations of $X_{4}$. However, since $h^{3,1}\left(X_{4}\right)=h^{1,1}\left(\tilde{X}_{4}\right)=4$ there are only four complex structure parameters rendering six of the $a_{i}$ redundant. It is also straightforward to compare $\tilde{m}_{1}, \tilde{m}_{6}$ for the fourfold $X_{4}$ with the corresponding threefold data in (4.11) and (4.12).

For the different phases we can identify the complex structure moduli in the hypersurface constraint by using the charge vectors $\ell^{(i)}$ in (4.22). For phase I one finds

$$
z_{1}^{\mathrm{I}}=\frac{a_{2} a_{4} a_{7}}{a_{1}^{2} a_{8}}, \quad z_{2}^{\mathrm{I}}=\frac{a_{1} a_{5}^{2} a_{6}^{3}}{a_{0}^{6}}, \quad z_{3}^{\mathrm{I}}=\frac{a_{3} a_{8}}{a_{1} a_{7}}, \quad z_{4}^{\mathrm{I}}=\frac{a_{7} a_{9}}{a_{1} a_{3}},
$$

while for the phase II one finds in accord with (4.23) that

$$
z_{1}^{\mathrm{II}}=z_{1}^{\mathrm{I}} z_{3}^{\mathrm{I}}, \quad z_{2}^{\mathrm{II}}=z_{2}^{\mathrm{I}} z_{3}^{\mathrm{I}}, \quad z_{3}^{\mathrm{II}}=\left(z_{3}^{\mathrm{I}}\right)^{-1}, \quad z_{4}^{\mathrm{II}}=z_{4}^{\mathrm{I}} z_{3}^{\mathrm{I}} .
$$

In order to compare to the threefold $Y_{3}$ we chose the gauge $a_{i}=1, i=2, \ldots, 6$ and $a_{8}=1$, such that

$$
a_{0}^{6}=\frac{1}{\left(z_{1}^{\mathrm{II}}\right)^{1 / 3} z_{2}^{\mathrm{II}} z_{3}^{\mathrm{II}}}, \quad a_{1}=\frac{1}{\left(z_{1}^{\mathrm{II}}\right)^{1 / 3}}, \quad a_{7}=z_{3}^{\mathrm{II}}\left(z_{1}^{\mathrm{II}}\right)^{1 / 3}, \quad a_{9}=\frac{z_{4}^{\mathrm{II}}}{\left(z_{1}^{\mathrm{II}}\right)^{2 / 3}} .
$$

It is straightforward to find the expression for phase I by inserting (4.33) into this expression for $a_{0}, a_{1}$ and $a_{7}, a_{9}$.

\footnotetext{
${ }^{10}$ The polynomial $\tilde{\mu}$ can be easily brought to the standard Weierstrass form by completing the square and the cube, i.e. $\tilde{y}=y+\frac{1}{2} \tilde{m}_{1} x z$ and $\tilde{x}=x-\frac{1}{12} \tilde{m}_{1}^{2} z^{2}$.
} 
Having determined the defining equations for the Calabi-Yau fourfolds it is straightforward to evaluate the discriminant $\Delta\left(X_{4}\right)$ of the elliptic fibration. Using (3.3) for a fourfold in the Weierstrass form (3.2) we find that

$$
\Delta\left(X_{4}\right)=-\tilde{m}_{6}\left(432 \tilde{m}_{6}+\tilde{m}_{1}^{6}\right) .
$$

We conclude that there will be seven-branes on the divisors $\tilde{m}_{6}=0$ and $432 \tilde{m}_{6}+\tilde{m}_{1}^{6}=0$ in the base $B_{3}^{X}$. The key observation is that in addition to a moduli independent part $\tilde{m}_{6}^{0}$ the full $\tilde{m}_{6}$ is shifted as

$$
\tilde{m}_{6}=\tilde{m}_{6}^{0}+a_{1}\left(u_{1} u_{2} u_{3} x_{1} x_{2} x_{3} x_{4} x_{5} x_{6}\right)^{6}+a_{7} u_{1}^{18} x_{1}^{24} x_{2}^{12} x_{3}^{6} x_{4}^{6}+a_{9} u_{1}^{6} u_{2}^{6} u_{3}^{6} x_{2}^{12} x_{5}^{12} x_{6}^{12} .
$$

The moduli dependent part is best interpreted in the phase II with $a_{1}, a_{7}$ and $a_{9}$ given in (4.34). In fact, when setting the fourth modulus to $z_{4}^{\mathrm{II}}=0$, one notes that the deformation of the seven-brane locus $\tilde{m}_{6}=0$ is precisely parameterized by $z_{3}^{\text {II }}$. By setting $x_{i}=1$ one fixes a point in the base of $X_{4}$ viewed as fibration with fiber $Y_{3}$. One is then in the position to compare the shift in (4.36) with the first constraint in (4.19) finding agreement if one identifies $\hat{z}_{1}=z_{3}^{\mathrm{II}}\left(z_{1}^{\mathrm{II}}\right)^{1 / 3}$. In the next section we will show that the open string BPS numbers of the local model with D5-branes of section 4.1 are recovered in the $z_{3}^{\text {II }}$ direction. The shift of the naive open modulus $\hat{z}_{1}$ by the closed complex structure modulus $z_{1}^{\mathrm{II}}$ fits nicely with a similar redefinition made for the local models in ref. [8]. This leaves us with the interpretation that indeed $z_{3}^{\text {II }}$ deforms the seven-brane locus and corresponds to an open string modulus in the local picture. As we will show in the next section, a $z_{3}^{\mathrm{II}}$-dependent superpotential is induced upon switching on fluxes on the sevenbrane. It can be computed explicitly and matched with the local results for D5-branes for an appropriate choice of flux.

A second interpretation of the shifts (4.36) by the monomials proportional to $z_{3}^{\mathrm{II}}, z_{4}^{\mathrm{II}}$ is via the heterotic dual theory on $Y_{3}$ and the spectral cover construction discussed in section 3.4. To see this, we bring $\tilde{\mu}$ into the form (3.19) by an appropriate coordinate redefinition. Setting $v=x_{1}^{6} x_{3}^{6} x_{4}^{6} x_{2}^{-6}, \tilde{u}_{1}=u_{1} x_{1} x_{2}, \tilde{u}_{2}=u_{2} x_{3}, \tilde{u}_{3}=u_{3} x_{4}$, and picking the local patch $x_{5}=x_{6}=1$ one rewrites (4.29) as

$$
\tilde{\mu}=p_{0}+v p_{+}+v^{-1} p_{-},
$$

where $p_{0}\left(y, x, z, \tilde{u}_{1}, \tilde{u}_{2}, \tilde{u}_{3}\right)=0$ is the threefold constraint (4.8) of $Y_{3}$, and

$$
p_{+}=\left(a_{7} \tilde{u}_{1}^{18}+a_{8} \tilde{u}_{1}^{6} \tilde{u}_{2}^{6} \tilde{u}_{3}^{6}\right) z^{6}, \quad p_{-}=a_{9} \tilde{u}_{1}^{6} \tilde{u}_{2}^{6} \tilde{u}_{3}^{6} z^{6} .
$$

Hence, in the local mirror limit in which $p_{-} \rightarrow 0$ [44], it is natural to interpret the modulus $z_{3}^{\mathrm{II}}$ as a bundle modulus of $V_{1}=\mathrm{SU}(1)$ in the heterotic dual theory, i.e. as a deformation of the spectral cover as in (3.19). One might be surprised that an SU(1)-bundle carries 
any bundle moduli due to the trivial structure group. Indeed the adequate physical interpretation of this configuration is in terms of heterotic five-branes as discussed in detail in [36].

Finally, as a side remark, let us note again that (4.35) with (4.30) and (4.31) is not the full answer for the discriminant since we have set many of the blow-up coordinates to unity. However, one can use the toric methods of [30, 32, 33] to determine the full minimal gauge group in the absence of flux to be

$$
G_{X_{4}}=E_{8}^{25} \times F_{4}^{69} \times G_{2}^{184} \times S U(2)^{276}
$$

Groups of such large rank are typical for elliptically fibered Calabi-Yau geometries with many Kähler moduli corresponding to blow-ups of singular fibers 33 .

\section{Mirror symmetry for Calabi-Yau fourfolds}

In this section we will describe mirror symmetry on fourfolds. In its weak formulation it states the equivalence of the complex structure moduli space of $X$ and the (instanton corrected) Kähler moduli space of its mirror $\tilde{X}$. As was pointed out in [49] this equivalence can be formulated in physical terms by considering topological field theories called the Aand B-model on the spaces $(\tilde{X}, X)$. These theories are consistent cohomological truncations of some particular $(2,2)$ superconformal field theories and their physical observables are the vertical subspace of the de Rham groups $H^{p, p}(\tilde{X}), 0 \leq p \leq n$ and the horizontal subspace of $H^{p}\left(X, \bigwedge^{q} T X\right), p+q=n$, respectively. In particular their marginal deformations coincide with the cohomology groups $H^{1,1}(\tilde{X})$ for the A-model and $H^{1}(X, T X)$ for the B-model that are, in geometrical terms, precisely the infinitesimal directions on the Kähler and complex structure moduli space of $\tilde{X}$ and $X$, respectively. Therefore, the physical statement of mirror symmetry is the equivalence of the A-model constructed from $\tilde{X}$ and the B-model constructed from $X$.

Our final goal is the calculation of special holomorphic quantities $F^{0}(\gamma)$ for the CalabiYau fourfolds. They are identified with the holomorphic superpotentials of $\mathcal{N}=1$ effective actions and at the same time they are generating functions of the genus zero Gromov-Witten invariants of the fourfold. In certain cases of special interest they can be further interpreted as generating functions of disk instantons in a dual type IIA theory. The (covariant) double derivatives of the $F^{0}(\gamma)$, w.r.t. to the moduli $t_{a}$ are three-point correlators $C_{a b \gamma}^{(1,1,2)}$. Their leading behaviour is fixed in the A-model by the classical intersection of two divisors with an element of $H_{4}(\tilde{X})$. In this sense $F^{0}(\gamma)$ is similar to the familiar prepotential $F^{0}$ of Calabi-Yau threefolds (2.3) whose triple derivative yields 
in the A-model a three-point function whose leading behaviour is fixed by the classical intersection between three divisors.

In order to understand the $F^{0}(\gamma)$ one needs to study more fundamental quantities, namely the two- and three-point correlation functions of the topological A- and B-model. They encode all other correlators of these topological theories.

As described in section 5.1 the operators representing elements of $H^{p}\left(X, \bigwedge^{q} T X\right)$ together with their two-point and three-point correlators, given by period integrals, form the B-model operator ring. In the large radius limit the A-model is defined by another ring, namely the vertical part of the classical cohomology ring $H^{* * *}(\tilde{X})$. Its two- and three-point correlators are simply given by the classical intersections. Away from the large radius point the notion of the classical intersection rings has to be extended to the quantum cohomology ring in which the three-point correlators are corrected by holomorphic instantons.

All these rings with two- and three-point correlators mentioned above carry a natural Frobenius structure, which we describe in section 5.2. Mirror symmetry identifies the quantum cohomology ring of the A-model with the B-model ring. For the precise identification one has to specify matching points in the moduli spaces. The natural candidate for our present purposes is the large radius point in the Kähler moduli space of the Amodel, which is identified with a point of maximal unipotent monodromy in the complex structure moduli space of the B-model. As described in section 5.3 the matching makes use of the mirror map, properties of the Picard-Fuchs system near the point of maximal unipotent monodromy and the classical intersection ring of $\tilde{X}$. The precise matching is necessary for the enumerative predictions of the A-model and the construction of a basis of the horizontal cohomology of $H^{2,2}(X)$, which is needed to identify the flux. It is crucial for this analysis to identify the integral basis of cohomology. One important step in this context is to determine the classical terms in the leading logarithmic period by means of analytic continuation to other points on the fourfold complex structure moduli space and a monodromy discussion.

After presenting the general formalism we discuss the relevant application to the case of elliptic Calabi-Yau fourfolds. In particular we will argue how the techniques and results of the first part help us finding the F-theory interpretation of the prepotentials $F^{0}(\gamma)$. These are precisely the quantities that we will identify with the flux superpotential (3.4) and we will furthermore understand its relation to the Type IIB superpotentials (3.5). We will exemplify this rather general analysis for our main example containing local $\mathbb{P}^{2}$ introduced in the last sections.

Since our discussion can at several points be generalized to arbitrary complex dimen- 
sional Calabi-Yau $n$-folds [37] we denote a mirror pair of Calabi-Yau $n$-folds by $\left(\tilde{X}_{n}, X_{n}\right)$ and identify with the fourfold case by putting $n=4$.

\subsection{States and correlation function of the B-model}

In the B-model one considers a family of $n$-folds $X_{z}$ fibered over the complex structure moduli space $\mathcal{M}, X_{z} \rightarrow \mathcal{M}$. The states 11 in the B-model are elements $B_{k}^{(j)}$ of the cohomology groups $H^{j}\left(X_{z}, \bigwedge^{j} T\right)$. Their cubic forms are defined as

$$
C\left(B_{a}^{(i)}, B_{b}^{(j)}, B_{c}^{(k)}\right)=\int_{X} \Omega\left(B_{a}^{(i)} \wedge B_{b}^{(j)} \wedge B_{c}^{(k)}\right) \wedge \Omega
$$

and vanish unless $i+j+k=n$. Here $\Omega$ is the unique holomorphic $(n, 0)$-form and $\Omega\left(B_{a}^{(i)} \wedge B_{b}^{(j)} \wedge B_{c}^{(j)}\right)$ is the contraction of the $n$ upper indices of $B_{a}^{(i)} \wedge B_{b}^{(j)} \wedge B_{c}^{(j)} \in$ $H^{n}\left(X_{z}, \wedge^{n} T X_{z}\right)$ with $\Omega$, which produces an anti-holomorphic $(0, n)$-form on $X_{z}$. Note that this is just the isomorphism $H^{i}\left(X_{z}, \bigwedge^{j} T X_{z}\right) \cong H^{n-j, i}\left(X_{z}\right)$ obtained by contraction with the holomorphic $(n, 0)$-form $\Omega$. We denote the image of $B_{k}^{(i)}$ in $H^{n-i, i}\left(X_{z}\right)$ by $b_{k}^{(i)}={ }^{\Omega}\left(B_{k}^{(i)}\right)$ and the inverse 12 by $B_{k}^{(i)}=\left(b_{k}^{(i)}\right)^{\Omega}$. Now we can define the hermitian metric

$$
G\left(B_{c}^{(i)}, \bar{B}_{d}^{(i)}\right)=\int_{X} b_{c}^{(i)} \wedge \bar{b}_{d}^{(i)}
$$

We consider only states $B_{a}^{(i)}$ for which the image $b_{a}^{(i)}$ is in the horizontal subspace $H_{H}^{n-j, i}\left(X_{z}\right)$ and assume that the $b_{a}^{(i)}$ form a basis of this space. For $B_{c}^{(1)} \in H^{1}\left(X_{z}, T X_{z}\right)$ the image spans all of $H^{n-1,1}\left(X_{z}\right)$ and $(5.2)$ is the Weil-Petersson metric on $\mathcal{M}$.

The integrals (5.1) and (5.2) are calculable by period integrals of the holomorphic $n$-form $\Omega$. It is very hard to integrate the periods directly. They encode however the variations of Hodge structure of the family $X_{z} \rightarrow \mathcal{M}$, which are reflected by the PicardFuchs differential equations on $\mathcal{M}$. The periods are therefore determined as solutions of the latter up to linear combination. The precise identification as addressed in section 5.3 is an important problem, as it determines the superpotential (3.4) of our F-theory setup.

For a given base point $z=z_{0}$ in the complex structure moduli space $\mathcal{M}$ with fiber $X_{z_{0}}$, one fixes a graded topological basis $\hat{\gamma}_{a}^{(p)}$ of the primary horizontal subspace $H_{H}^{n}\left(X_{z_{0}}, \mathbb{Z}\right)$. Here $a=1, \ldots, h_{H}^{n-p, p}$ labels the basis $\hat{\gamma}_{a}^{(p)}$ for fixed $p=0, \ldots, n$ of each graded piece $H^{n-p, p}\left(X_{z}\right)$. These forms can be chosen to satisfy (3.10) in addition 13 . Later on in section

\footnotetext{
${ }^{11}$ We use in the following the same symbol for states and operators.

${ }^{12}$ The inversion is just the contraction $\left(b_{k}^{(i)}\right)^{\Omega}=\frac{1}{\|\Omega\|^{2}} \bar{\Omega}^{a_{1} \ldots a_{n-i} b_{1} \ldots b_{i}}\left(b_{k}^{(i)}\right)_{a_{1} \ldots a_{n-i} \bar{b}_{1} \ldots \bar{b}_{i}}$ such that $(\Omega)^{\Omega}=$ 1. Formally it is the multiplication with the inverse $\mathcal{L}^{-1}$ of the Kähler line bundle $\mathcal{L}=\langle\Omega\rangle$, see e.g. 550 . ${ }^{13}$ The generalization from fourfolds to $n$-folds is the obvious one.
} 


\begin{tabular}{|c|c|c|c|c|c|}
\hline Dimension & 1 & $h^{3,1}(X)$ & $h^{2,2}(X)$ & $h^{3,1}(X)$ & 1 \\
\hline Basis of & $\hat{\gamma}_{a_{0}}^{(0)}$ & $\hat{\gamma}_{a}^{(1)}$ & $\hat{\gamma}_{\beta}^{(2)}$ & $\hat{\gamma}_{a}^{(3)}$ & $\hat{\gamma}_{a_{0}}^{(4)}$ \\
$H_{H}^{4}(X, \mathbb{Z})$ & $\beta_{a_{0}}^{(0)}$ & $\beta_{a}^{(1)}$ & $\beta_{\beta}^{(2)}$ & $\beta_{a}^{(3)}$ & $\beta_{a_{0}}^{(4)}$ \\
\hline
\end{tabular}

Table 5.1: Topological $\hat{\gamma}$ and moduli-dependent $\beta$ basis of $H_{H}^{4}(X, \mathbb{Z})$

5.3 we will identify this grading by $p$ with the natural grading on the observables of the A-model which are given by the vertical subspaces $H_{V}^{p, p}(\tilde{X})$ of the mirror cohomology.

Note that the $\hat{\gamma}_{a}^{(p)}$ basis serves as a local frame of the vector bundle $\mathcal{H}_{H}^{n}(X) \rightarrow \mathcal{M}$ over the moduli space whose fiber at the point $z \in \mathcal{M}$ is $H_{H}^{n}\left(X_{z}\right)$. However, the individual $H^{n, n-p}\left(X_{z}\right)$ form no holomorphic vector bundles over $\mathcal{M}$ since holomorphic and antiholomorphic coordinates are mixed under a change of complex structure $z$. Only the horizontal parts of $F^{k}=\oplus_{p=0}^{k} H^{n-p, p}\left(X_{z}\right)$ form holomorphic vector bundles for which we introduce frames $\beta_{a}^{(k)}$ specified by the basis expansion

$$
\beta_{a}^{(k)}=\hat{\gamma}_{a}^{(k)}+\sum_{p>k} \Pi_{a}^{(p, k) c} \hat{\gamma}_{c}^{(p)}
$$

In special coordinates $t^{a}$ at the point of maximal unipotent monodromy this can be written as $\left\{\beta^{(0)}=\Omega, \beta_{a}^{(1)}=\partial_{a} \Omega, \ldots\right\}$, cf. (5.6). We note that for this basis choice we fixed the overall normalization of each $\beta_{a}^{(k)}$ such that the coefficient of $\hat{\gamma}_{a}^{(k)}$ is unity. This is needed to obtain the right inhomogeneous flat coordinates on $\mathcal{M}$ and to make contact with enumerative predictions for the A-model, see section 5.3. For grade $k=0$ it corresponds to the familiar gauge choice of $\Omega$ in mirror applications to threefolds, see (5.6). We also introduce a basis of integral homology cycles $\gamma_{a}^{(p)}$ dual to $\hat{\gamma}_{a}^{(p)}$ as in (3.9). Then, by construction of the basis (5.3), the period matrix $P$ defined by period integrals takes an upper triangular form in this basis,

$$
P=\int_{\gamma_{a}^{(p)}} \beta_{b}^{(k)}= \begin{cases}\Pi_{b}^{(p, k) a}, & p>k \\ \delta_{a b}, & p=k \\ 0, & p<k\end{cases}
$$

where $(p, k)$ is the bi-grade of the non-trivial periods $\Pi_{b}^{(p, k) a}$. Since $\hat{\gamma}_{a}^{(p)}$ are topological and thus are locally constant on $\mathcal{M}$, the moduli dependence of (5.3) is captured by the moduli dependence of the period matrix $P \equiv P(z)$. For Calabi-Yau fourfolds we summarize the basis choices and the periods in Table 5.1. The $(n, 0)$-form $\Omega$ can always be expanded over the topological basis $\hat{\gamma}_{a}^{(p)}$ of $H_{H}^{n}(X, \mathbb{Z})$ as

$$
\Omega=\Pi_{1}^{(p, 0) a} \hat{\gamma}_{a}^{(p)} \equiv \Pi^{(p) a} \hat{\gamma}_{a}^{(p)}
$$


where we introduced a simplified notation $\Pi^{(p) a}$ for the periods $\Pi_{1}^{(p, 0) a}$ of the holomorphic $n$-form already given in (3.8). For an arbitrarily normalized $n$-form $\Omega$, the periods $\left(X^{0}, X^{a}\right)=\left(\Pi^{(0) 1}, \Pi^{(1) a}\right)$ with $a=1, \ldots, h^{n, 1}\left(X_{z_{0}}\right)$ at the fixed reference point $z_{0}$ form for all Calabi-Yau $n$-folds homogeneous projective coordinates of the complex moduli space $\mathcal{M}$. The choice of inhomogeneous coordinates set by

$$
t^{a}=\frac{X^{a}}{X^{0}}=\frac{\int_{\gamma_{a}^{(1)}} \Omega}{\int_{\gamma_{1}^{(0)}} \Omega},
$$

which agrees with the basis choice in (5.3), corresponds to a gauge in the Kähler line bundle $\langle\Omega\rangle$. At the point of maximal unipotent monodromy $X^{0}$ and $X^{a}$ are distinguished by their monodromy, as $X^{0}$ is holomorphic and single-valued and $X^{a} \sim \log (z)$ has monodromy $X^{a} \mapsto X^{a}+1$. Below the $t^{a}$ in (5.6) are identified with the complexified Kähler parameters of the mirror $\tilde{X}$.

The $t^{a}$ defined in (5.6) are flat coordinates for the Gauss-Manin connection $\nabla_{a}$, i.e. the latter becomes just the ordinary differential $\frac{\partial}{\partial t_{a}}$. This can be seen from the gauge choice reflecting in the basis (5.3) combined with the Griffiths transversality constraint $\nabla_{a}\left(F^{k}\right) \subset F^{k+1} / F^{k}$ which together imply [37] $\nabla_{a} t^{b}=\delta_{a}^{b}$, i.e $\nabla_{a}=\partial_{a}$. In these coordinates the three-point coupling becomes

$$
C_{a b c}^{(1, k, n-k-1)}=C\left(\left(\beta_{a}^{(1)}\right)^{\Omega},\left(\beta_{b}^{(k)}\right)^{\Omega},\left(\beta_{c}^{(n-k-1)}\right)^{\Omega}\right)=\int_{X} \beta_{c}^{(n-k-1)} \wedge \partial_{a} \beta_{b}^{(k)} .
$$

This triple coupling is a particularly important example of (5.1). Here we use (5.3) and the fact that $\left(\beta_{a}^{(1)}\right)^{\Omega} \wedge\left(\beta_{b}^{(k)}\right)^{\Omega}$ can be replaced by $\left(\nabla_{a} \beta_{b}^{(k)}\right)^{\Omega}$ [37] under the integral (5.1) to obtain (5.7). Furthermore, one can show from the properties of the Frobenius algebra that all other triple couplings in (5.1) can be expressed in terms of (5.7), see section 5.2 .

The holomorphic topological two-point couplings of (3.10) in this basis trivially read

$$
\eta_{a b}^{(k)}=\int_{X} \beta_{b}^{(n-k)} \wedge \beta_{a}^{(k)}
$$

since only the lowest $\hat{\gamma}^{(p)}$ for $p=k$ in the upper-triangular basis transformation (5.3) contributes to the integral due to the second property of (3.10). In particular $\eta^{(k)}$ is moduli independent. From the above it is easy to see the basis expansion at grade $k+1$,

$$
\partial_{a} \beta_{b}^{(k)}=C_{a b c}^{(1, k, n-k-1)} \eta_{(n-k-1)}^{c d} \beta_{d}^{(k+1)},
$$

where $\eta_{(p)}^{c d}$ is the inverse of $\eta_{c d}^{(p)}$.

Let us finish this section with some comments on general properties of the periods and their implications on the $\mathcal{N}=1$ effective action. The period integrals (5.4) obey 
differential and algebraic relations, which are different from the special geometry relations of Calabi-Yau threefold periods. They have however exactly the same origin namely the Griffiths transversality constraints $\int_{X} \Omega \wedge \partial_{i_{1}} \ldots \partial_{i_{k}} \Omega=0$ for $k<n$. However since $a \wedge b=$ $(-1)^{n} b \wedge a$ for $a, b$ real $n$-forms one has additional algebraic relations from $\int_{X} \Omega \wedge \Omega=0$ between the periods $\Pi^{(p) a}$ for $n$ even like $n=4$, which are absent for $n$ odd, in particular the threefold case. $\mathcal{N}=2$ compactifications of type II on Calabi-Yau threefolds to four dimensions inherit the structure induced by the above differential relations in the vector moduli space. In fact such a structure is, up to minor generalizations, generic to $\mathcal{N}=2$ supergravity theories and known as special Kähler geometry. For $\mathcal{N}=1$ supergravity theories in $4 \mathrm{~d}$ there is generically no special structure beyond Kähler geometry.

\subsection{The Frobenius Algebras}

As was mentioned above the B-model operators form a Frobenius algebra. Since also the A-model classical cohomology operators as well as its quantum cohomology operators form such an algebra it is worthwhile to describe the general structure before discussing the precise matching in the next section.

A Frobenius algebra has the following structures. It is a graded vector space $\mathcal{A}=$ $\oplus_{i=1}^{n} \mathcal{A}^{(i)}$ with $\mathcal{A}^{(0)}=\mathbb{C}$ equipped with a non-degenerate symmetric bilinear form $\eta$ and a cubic form

$$
C^{(i, j, k)}: \mathcal{A}^{(i)} \otimes \mathcal{A}^{(j)} \otimes \mathcal{A}^{(k)} \rightarrow \mathbb{C}
$$

$i, j, k \geq 0$ and the following properties:

(F1) Degree: $C^{(i, j, k)}=0$ unless $i+j+k=n$

(F2) Unit: $C_{1 b c}^{(0, i, j)}=\eta_{b c}^{(i)}$

(F3) Nondegeneracy: $C^{(1, i, j)}$ is non-degenerate in the second slot

(F4) Symmetry: $C_{a b c}^{(i, j, k)}=C_{\sigma(a b c)}^{(\sigma(i, j, k))}$ under any permutation of the indices.

(F5) Associativity: $C_{a b p}^{(i, j, n-i-j)} \eta_{(n-i-j)}^{p q} C_{q e f}^{((i+j), k,(n-i-j-k))}=C_{a e q}^{(i, k, n-i-k)} \eta_{(n-i-k)}^{q p} C_{p b f}^{(i+k, j, n-i-j-k)}$ where the sum over common indices is over a basis of the corresponding spaces.

The product

$$
\mathcal{O}_{a}^{(i)} \cdot \mathcal{O}_{b}^{(j)}=C_{a b q}^{(i, j, i+j)} \eta_{(i+j)}^{q p} \mathcal{O}_{p}^{(i+j)}
$$

defines now the Frobenius algebra for a basis of elements $\mathcal{O}_{k}^{(i)}$ of $\mathcal{A}^{(i)}$ which is easily seen using (F4) to be commutative. Note that the associativity implies that $n$-point 
correlators can be factorized in various ways in the three-point functions. Also the threepoint correlators are not independent and by associativity, non-degeneracy and symmetry it can be shown 14 that all three-correlators can be expressed in terms of the $C^{(1, r, n-r-1)}$ correlators defined in (5.7)

It is easy to see that the $B^{(i)}$ operators of the B-model with the correlators defined by (5.1) or equivalently (5.7) and (5.8) fulfill the axioms of a Frobenius algebra.

Let us consider the A-model operators. As already mentioned such an operator corresponds to an element in the vertical subspace $H_{V}^{*, *}(\tilde{X})$. It is generated by the Kähler forms $J_{i}, i=1, \ldots, h^{1,1}(\tilde{X})$ and naturally graded,

$$
A_{\alpha}^{(p)}=a_{\alpha}^{i_{1}, \ldots, i_{p}} J_{i_{1}} \wedge \ldots \wedge J_{i_{p}} \in H^{p, p}(\tilde{X})
$$

For the classical A-model the correlation functions are simply the intersections

$$
C_{a b c}^{0(i, j, k)}=C\left(A_{a}^{(i)} A_{b}^{(j)} A_{c}^{(k)}\right)=\int_{\tilde{X}} A_{a}^{(i)} \wedge A_{b}^{(j)} \wedge A_{c}^{(k)}
$$

They vanish unless $i+j+k=n$. The topological metric is similarly defined by

$$
\eta_{a b}^{(k)}=\int_{\tilde{X}} A_{a}^{(k)} \wedge A_{b}^{(n-k)}
$$

and together with (5.13) this defines a Frobenius algebra. Clearly the $A_{k}^{(p)}$ are not freely generated by the $J_{i}$. The products $J_{i_{1}} \wedge \ldots \wedge J_{i_{p}}$ are set to zero if their pairings (5.14) with all other cohomology elements vanish. This is easily calculated using toric techniques and reflects geometrical properties of $\tilde{X}$ like for instance fibration structures.

The classical intersections are extended using mirror symmetry to the quantum cohomological intersections 15

$$
C\left(A_{a}^{(i)} A_{b}^{(j)} A_{c}^{(k)}\right)=C_{a b c}^{0(i, j, k)}+\text { instanton corrections }
$$

where the instanton corrections are from holomorphic curves with meeting conditions on the homology cycles dual to the $A_{k}^{(p)}$. They are such that the correlator vanishes again unless $i+j+k=n$. Note that the $C\left(A_{a}^{(i)} A_{b}^{(j)} A_{c}^{(k)}\right)$ depend via the instantons on the complexified Kähler parameters of $\tilde{X}$, while $\eta_{a b}^{(k)}=\int_{\tilde{X}} A_{a}^{(k)} \wedge A_{b}^{(n-k)}$ is still purely topological. There are no instanton corrections present because in the moduli space for the two-pointed sphere not all zero modes are saturated due to the one remaining conformal Killing field on the sphere.

\footnotetext{
${ }^{14}$ See $[25$ for an explicit inductive proof.

${ }^{15}$ We denote the both the operators of the classical algebra and the operators of quantum cohomology algebra by $A_{k}^{(p)}$.
} 


\subsection{Matching of the A-model and B-model Frobenius algebra}

We now describe the matching of the B-model Frobenius algebra with the $A$ model quantum Frobenius algebra. At the large radius point of the Kähler structure, the correlation functions of the classical $A$-model can be calculated using toric intersection theory. We will match this information with the leading logarithmic behaviour of the periods at the corresponding point in the complex structure moduli space, the point of large complex structure, which is characterized by its maximal logarithmic degeneration, which leads to a maximal unipotent monodromy.

Let us now discuss the Picard-Fuchs differential operators associated to the mirror Calabi-Yau $X$ at the large complex structure point. Since we are dealing throughout our paper with mirror pairs $\left(\tilde{X}_{4}, X_{4}\right)$ that are given torically, the derivation of the PicardFuchs operators simplifies significantly, since they are completely encoded by the toric data. To the Mori cone generators $\ell^{(a)}$ on the A-model side one associates the canonical GKZ-system of differential operators on the B-model side

$$
\mathcal{D}_{a}=\prod_{\ell_{i}^{(a)}>0}\left(\frac{\partial}{\partial a_{i}}\right)^{\ell_{i}^{(a)}}-\prod_{\ell_{i}^{(a)}<0}\left(\frac{\partial}{\partial a_{i}}\right)^{-\ell_{i}^{(a)}},
$$

where the derivative is taken with respect to the coefficients $a_{i}$ of monomials in the constraint $f=0$ defining $X$. By the methods described in [21] we obtain linear Picard-Fuchs operators $\mathcal{L}_{a}(\underline{\theta}, \underline{z})$, which are written in terms of the logarithmic derivatives $\theta_{a}=z_{a} \frac{\partial}{\partial z_{a}}$ with respect to the canonical complex variables $z_{a}$ that vanish at the large complex structure point defined by (4.9). We extract the leading $\theta$ piece of the differential operators as the formal $\operatorname{limit} \mathcal{L}_{i}^{\lim }(\underline{\theta})=\lim _{z_{i} \rightarrow 0} \mathcal{L}_{i}(\underline{\theta}, \underline{z}), i=1, \ldots, r$ and consider the algebraic ring

$$
\mathcal{R}=\mathbb{C}[\underline{\theta}] /\left(\mathcal{J}=\left\{\mathcal{L}_{1}^{\lim }, \ldots, \mathcal{L}_{r}^{\lim }\right\}\right)
$$

This ring is graded by the degree $k$ in $\theta$ and we denote the ring at grade $k$ by $\mathcal{R}^{(k)}$ whose number of elements is given by $h_{n-k, k}^{H}(X)=h_{k, k}^{V}(\tilde{X})$ for $k=0, \ldots, n$. We note that for $k=0,1, n-1, n$ there is no difference between the vertical (horizontal) homology and the full homology groups. Let us explain in more detail how this ring connects the $A$ and the B-model structure at large radius:

(A) The construction of the ring is up to normalization equivalent to the calculation of the intersection numbers of the $A$-model. In particular the $n$-point intersections appear as coefficients of the up to a normalization unique top ring element $\mathcal{R}^{(n)}=\sum_{i_{1} \leq \ldots \leq i_{n}} C_{i_{1}, \ldots, i_{n}}^{0} \theta_{i_{1}} \ldots \theta_{i_{n}}$ and similar the $\mathcal{R}^{(k)}$ are generated by $\mathcal{R}_{\alpha}^{(k)}=\sum_{i_{1} \leq \ldots \leq i_{k}} a_{\alpha}^{i_{1}, \ldots, i_{k}} \theta_{i_{1}} \ldots \theta_{i_{k}}, \alpha=1, \ldots, h_{k, k}^{V}(\tilde{X})$, where $a_{\alpha}^{i_{1}, \ldots, i_{k}} \kappa_{i_{1}, \ldots, i_{k}, j_{1}, \ldots j_{n-k}}=$ $C_{\alpha, j_{1}, \ldots j_{n-k}}^{0}$. 
(B) The ring $\mathcal{R}^{(k)}$ is in one-to-one correspondence to the solutions to the Picard-Fuchs equations at large radius. As discussed before the solutions are characterized by their monodromies around this point, i.e. they are graded by their leading logarithmic structure. To a given ring element $\mathcal{R}^{(k) a}=\sum_{|\underline{\alpha}|=k} \frac{1}{(2 \pi i)^{k}} m_{\underline{\alpha}}^{a} \theta_{1}^{\alpha_{1}} \ldots \theta_{h}^{\alpha_{h}}$ in $\mathcal{R}^{(k)}$ we associate a solution of the form

$$
\tilde{\mathcal{R}}^{(k) a}=X_{0}(\underline{z})\left[\mathbb{L}^{(k) a}+\mathcal{O}\left(\log (z)^{|\alpha|-1}\right)\right],
$$

with leading logarithmic piece of order $k$,

$$
\mathbb{L}^{(k) a}=\sum_{|\underline{\alpha}|=k} \frac{1}{(2 \pi i)^{k}} \tilde{m}_{\underline{\alpha}}^{a} \log ^{\alpha_{1}}\left(z_{1}\right) \ldots \log ^{\alpha_{h}}\left(z_{h}\right),
$$

by assigning $\tilde{m}_{\underline{\alpha}}^{a}\left(\prod_{i} \alpha_{i} !\right)=m_{\underline{\alpha}}^{a}$. In particular we map the unique element 1 of $\mathcal{R}^{(0)}$ to the unique holomorphic solution $X_{0}(\underline{z})=1+\mathcal{O}(z)$. The above map follows from the fact that all $\mathcal{L}_{s}^{\lim }$ in the ideal $\mathcal{J}$ must annihilate the leading logarithmic terms for $\Pi^{(k) a}$ to be a solution which yields the same conditions as for $\mathcal{R}^{(k)}$ to be normal to $\mathcal{J}$ in (5.17).

The two facts (A) and (B) imply mirror symmetry at the level of the classical couplings and can be proven for toric hypersurfaces by matching the toric intersection calculation with the toric derivation of the Picard-Fuchs operators as it was argued in the threefold case [51]. The identification

$$
\theta_{i} \leftrightarrow J_{i}
$$

provides a map between $\mathcal{R}_{a}^{(k)}$ and the classical A-model operators $A_{a}^{(k)}$ defined in (5.12). This provides also the matching of the A- and B-model Frobenius structures at the large radius limit by identifying the periods of $\Omega$ and the solutions of the Picard-Fuchs system in the following way. To a given element $\mathcal{R}_{a}^{(k)}$ we can associate an A-model operator $A_{a}^{(k)}$ by the replacement (5.20) and wedging of the $J_{i}$. Similarly we can construct the dual B-model operators $\beta_{a}^{(k)}$ in $F^{k}$ by applying the elements of the ring $\mathcal{R}^{(k)}$ as differentials in the $z_{i}$ to the holomorphic form $\Omega$. We obtain the map

$$
\left.A_{a}^{(k)} \mapsto \beta_{a}^{(k)}\right|_{z=0}=\left.\mathcal{R}_{a}^{(k)} \Omega\right|_{z=0} .
$$

This determines the Frobenius algebra of the B-model completely. However, to relate the two- and three-point functions to the period integrals of the $\beta_{a}^{(k)}$ along the lines of section 5.1 we have to specify the topological basis $\gamma_{a}^{(k)}$ in terms of the A-model operator $A_{a}^{(k)}$ as well. First we select a basis of solutions $\Pi^{(k) a}(z)$ of the Picard-Fuchs system with leading logarithm $\mathbb{L}^{(k) a}$ that is dual to the $A_{a}^{(k)}$ at large radius, i.e.

$$
\mathcal{R}_{a}^{(k)} \mathbb{L}^{(k) b}=\delta_{a}^{b},
$$


in the limit $z_{i} \rightarrow 0$ [38]. Then, the $\gamma_{a}^{(k)}$ are fixed by setting the periods $\Pi_{1}^{(k, 0) a} \equiv \Pi^{(k) a}(z)$ in the expansion (5.5) of $\Omega=\Pi^{(k) a}(z) \hat{\gamma}_{a}^{(k)}$ that provides a map between the $\mathbb{L}^{(k) a}$ and $\hat{\gamma}_{a}^{(k)}$. With these definitions the requirements (5.4) on the upper triangular basis $\beta_{a}^{(k)}$ are trivially fulfilled since $\beta_{b}^{(q)}=\mathcal{R}_{b}^{(q)} \Omega=\mathcal{R}_{b}^{(q)}\left(\Pi^{(q) a} \hat{\gamma}_{a}^{(q)}+\ldots\right) \rightarrow \hat{\gamma}_{b}^{q}+\ldots$ where the dots indicate forms $\hat{\gamma}_{a}^{(k)}$ at grade $k>q$ with higher logarithms.

Let us exploit this matching by e.g. considering the B-model coupling $C_{a b c}^{(1,1, n-2)}$. We obtain in (5.7) for fourfolds that

$$
C_{a b \gamma}^{(1,1,2)}=\partial_{t^{a}} \Pi_{b}^{(2,1) \delta} \eta_{\delta \gamma}^{(2)}=\partial_{t^{a}} \partial_{t^{b}} \Pi^{(2) \delta} \eta_{\delta \gamma}^{(2)}=: \partial_{t^{a}} \partial_{t^{b}} F^{g=0}(\gamma),
$$

where $a, b=1, \ldots, h^{3,1}$ and $\gamma=1, \ldots, h_{2,2}^{H}$. Here we used the upper triangular form (5.3) of $\beta_{a}^{(k)}$ and the intersection properties (3.10) of the $\hat{\gamma}_{i}$ for the first equality. Then we replaced $\partial_{t_{a}} \beta^{(0)}=\beta_{a}^{(1)}$ for general dimension $n$ as follows from (5.9) and property (F2) in flat coordinates. If we now let $z_{i} \rightarrow 0$ and use the flat coordinates (5.6), which are given by (5.18) as $t^{i} \sim \log \left(z_{i}\right)+$ hol. $\rightarrow \log \left(z_{i}\right)$, we see that in this limit the classical intersection $C_{a b \gamma}^{0(1,1,2)}$ of (5.13) are reproduced. Once the matching is established in this large radius limit we can define the full quantum cohomological Frobenius structure by (5.23) in the coordinates (5.6). The latter can be viewed as the classical topological intersections deformed by instanton corrections.

For the case at hand the intersections are obtained from the second derivative of the holomorphic quantities $F^{0}(\gamma)$ that were introduced in (5.23) for each basis element $\beta_{\gamma}^{(2)}, \gamma=1, \ldots h_{H}^{2,2}\left(X_{4}\right)$. These are the analogues of the holomorphic prepotential $F^{0}$ familiar from the threefold case and they are obtained in the general discussion of section 6 from the generating functional: 16 (6.4) for $k=1$. The relation (5.23) tells us that we obtain them simply from the Picard-Fuchs equation as double-logarithmic solutions, that we will identify below. However as mentioned above the identification using the ring structure fixes the solutions of the Picard-Fuchs system so far only up to normalizations. The normalization of the unique holomorphic solution is determined by the fact that the leading term in $X_{0}(z)=1+\mathcal{O}(z)$ has to be one. Also the dual period can be uniquely normalized by the classical $n$-point intersections. The single logarithmic solutions are normalized to reproduce the effect of a shift of the NS-background field $B$ on $t^{i}=$ $\int_{\mathcal{C}}(B+i \omega)$, where $\mathcal{C}$ is a generator of $H_{2}(\tilde{X}, \mathbb{Z})$ and $\omega$ is the Kähler form. The shift is then $t^{i} \rightarrow t^{i}+1$. This corresponds to the monodromy around $z_{i}=0$ and implies according to (5.6) that $\tilde{m}_{\underline{\alpha}}^{a}=1$ for $|\alpha|=1$ in (5.18) .

All further $t$-dependent quantities are restricted further by the monodromy of the period vector $\Pi$ of the holomorphic $(4,0)$-form, $\Pi=\left(\Pi^{(0)}, \Pi^{(1) *}, \Pi^{(2) *}, \Pi^{(3) *}, \Pi^{(4)}\right)^{T}$ around

\footnotetext{
${ }^{16}$ We note that the terms $b_{a \gamma}^{0}, a_{\gamma}^{0}$ are irrelevant for the quantum cohomology, but important for the large radius limit of the superpotential (3.4).
} 
$z_{i}=0$. Let $\Sigma$ be the matrix representing the intersection $K=\int_{X} \Omega \wedge \bar{\Omega}=\Pi \Sigma \Pi^{\dagger}$. Using (5.5, 3.10) it is easy to see that the anti-diagonal of $\Sigma$ is given by the blocks $\left(1,\left(\eta^{(1)}\right)^{T}, \eta^{(2)}, \eta^{(1)}, 1\right)$. The monodromies act by $\Pi \rightarrow M_{i} \Pi$, where $M_{i}$ is a $\left(h_{4}^{H} \times h_{4}^{H}\right)$ matrix. The monodromy invariance of $K$ and $\hat{\gamma}_{a}^{(p)} \in H_{H}^{4}\left(X_{4}, \mathbb{Z}\right)$ implies

$$
M_{i}^{T} \Sigma M_{i}=\Sigma
$$

with $M_{i}$ an integer matrix. Using the monodromy at other points in the moduli space and analytic continuation, one can fix all a priori undetermined constants in the solutions to the Picard-Fuchs system.

However, this is tedious and useful information about some of the irrational constants that appear e.g. in the leading logarithmic solution follow from the Frobenius method [51, 52. By this method the leading logarithmic solution can be obtained by applying the operator $D^{(4)}=\frac{1}{4 !(2 \pi i)^{4}} \mathcal{K}_{i_{1} i_{2} i_{3} i_{4}} \partial_{\rho_{i_{1}}} \partial_{\rho_{i_{2}}} \partial_{\rho_{i_{3}}} \partial_{\rho_{i_{4}}}$ on the fundamental solution

$$
\omega_{0}(\underline{z}, \underline{\rho})=\sum_{\underline{n}} c(\underline{n}, \underline{\rho}) z^{\underline{n}+\underline{\rho}}
$$

with

$$
c(\underline{n}, \underline{\rho})=\frac{\Gamma\left(-\sum_{\alpha} l_{0}^{(\alpha)}\left(n_{\alpha}+\rho_{\alpha}\right)+1\right)}{\prod_{i} \Gamma\left(\sum_{\alpha} l_{i}^{(\alpha)}\left(n_{\alpha}+\rho_{\alpha}\right)+1\right)}
$$

and setting $\underline{\rho}=0$. The general leading logarithmic solution, i.e. with all possible admixtures of lower logarithmic solutions, for $X^{0}=\left.\omega_{0}(\underline{z})\right|_{\underline{\rho}=0}$ reads

$$
\Pi^{(4)}=X^{0}\left(\frac{1}{4 !} \mathcal{K}_{i j k l} t^{i} t^{j} t^{k} t^{l}+\frac{1}{3 !} a_{i j k} t^{i} t^{j} t^{k}+\frac{1}{2 !} a_{i j} t^{i} t^{j}+a_{i} t^{i}+a_{0}\right),
$$

where as in the threefold case $\mathcal{K}_{i j k l}$ is the classical top intersection. It was observed in [51] for the threefold case that the Frobenius method reproduces some of the topological constants in (2.3) $)$. In particular $\int_{\tilde{Y}_{3}} c_{2} \wedge J_{i}=\frac{3}{\pi^{2}} \mathcal{K}_{i j k} \partial_{\rho_{j}} \partial_{\rho_{k}} c(\underline{0}, \underline{\rho})_{\underline{\rho}=0}$ and $\int_{\tilde{Y}_{3}} c_{3}=$ $\frac{1}{3 ! \zeta(3)} \mathcal{K}_{i j k} \partial_{\rho_{i}} \partial_{\rho_{j}} \partial_{\rho_{k}} c(\underline{0}, \underline{\rho})_{\underline{\rho}=0}$, where we write $c_{i}$ for the $i$-th Chern class of the Calabi-Yau manifold. If we generalize these to fourfolds, we get

$$
\int_{\tilde{X}_{4}} \frac{3}{4} c_{2}^{2}+c_{4}=\frac{1}{4 ! \zeta(4)} \mathcal{K}_{i j k l} \partial_{\rho_{i}} \partial_{\rho_{j}} \partial_{\rho_{k}} \partial_{\rho_{l}} c(\underline{0}, \underline{\rho})_{\underline{\rho}}=0
$$

These constants are expected to appear as coefficients of the lower order logarithmic solutions in (5.27). Similar as in the threefold case one can also use the induced Ktheory charge formula [53, 54] in combination with central charge formula with $A$ being the bundle on the brane wrapping $\tilde{X}_{4}$

$$
\vec{Q} \cdot \vec{\Pi}=-\int_{\tilde{X}_{4}} e^{-J} \operatorname{ch}(A) \sqrt{\operatorname{td}\left(\tilde{X}_{4}\right)}=Z(A)
$$


and mirror symmetry to obtain information about the subleading logarithmic terms in the periods.

Let us apply a more direct argument and use properties of the simplest Calabi-Yau fourfold, the sextic in $\mathrm{P}^{5}$. The mirror has the Picard-Fuchs equation (see e.g. [55])

$$
\theta^{5}-6 z \prod_{k=1}^{5}(6 \theta+k)
$$

We can easily construct solutions at $z=0$ using the Frobenius method, but let us first give a different basis of logarithmic solutions namely

$$
\hat{\Pi}_{k}=\frac{1}{(2 \pi i)^{k}} \sum_{l=0}^{k}\left(\begin{array}{l}
k \\
l
\end{array}\right) \log (z)^{l} s_{k-l}(z)
$$

where

$$
\begin{aligned}
X^{0} & =s_{0}=1+720 z+748440 z^{2}+\ldots, \quad s_{1}=6246 z+7199442 z^{2}+\ldots, \\
s_{2} & =20160 z+327001536 z^{2}+\ldots, \quad s_{3}=-60480 z-111585600 z^{2}+\ldots \\
s_{4} & =-2734663680 z^{2}-57797926824960 z^{3}+\ldots .
\end{aligned}
$$

The point is that under the mirror map one obtains $\hat{\Pi}_{k}=t^{k}+\mathcal{O}(q)$, so that these solutions correspond to the leading volume term of branes of real dimension $2 k$. The "conifold" locus of the sextic is at $\Delta=1-6^{6} z=0$. Near that point the Picard-Fuchs equation has the indicials $\left(0,1,2,3, \frac{3}{2}\right)$. It is easy to construct solutions and we choose a basis in which the solution to indicial $k \in \mathbb{Z}$ has the next power $z^{4}$. The only unique solution is the one with the branch cut

$$
\nu=\Delta^{\frac{3}{2}}+\frac{17}{18} \Delta^{\frac{5}{2}}+\frac{551}{648} \Delta^{\frac{7}{2}}+\ldots .
$$

The situation at the universal conifold is crucial for mirror symmetry in various dimensions $n$. At this point the non-trivial monodromy is between a cycle of topology $\mathbb{T}^{n}$ that corresponds to the solution $X^{0}$, i.e. the zero-dimensional brane in the $A$-model, which is uniquely defined at $z=0$, and a cycle of topology $\mathbb{S}^{n}$ that corresponds to the solution $\Pi^{(n)}$, i.e. the top dimensional brane in the $A$-model. The topological intersection between these cycles is 1 and their classes in the homology are the fiber and the base of the SYZ-fibration respectively [28]. In odd dimensional Calabi-Yau manifolds the conifold monodromy acts on the vector $\Pi_{\text {red }}=\left(\Pi^{(n)}, X_{0}\right)^{T}$ as $M_{2 \times 2}=\left(\begin{array}{ll}1 & 0 \\ 1 & 1\end{array}\right)$. This corresponds to the Lefschetz formula with vanishing $\Pi^{(n)}$, i.e. the quantum volume of $\tilde{X}$ vanishes. 
In four dimensions we have a monodromy of order two and the only way to write an integral idempotent monodromy compatible with the intersection (5.24) is $M_{2 \times 2}=$ $\left(\begin{array}{ll}0 & 1 \\ 1 & 0\end{array}\right)$. It is noticeable that the zero- and the highest dimensional brane get exchanged by the conifold monodromy in even dimensions.

This implies that $X^{0}=\eta-c \nu$ and $\Pi^{17}=\eta+c \nu$. Here $\eta$ is a combination of solutions at $\Delta=0$ without branch cut. We can determine the latter by analytic continuation of $X^{0}$ to the conifold. While the precise combination is easily obtained, the only constant that matters below is $c$, which turns out to be $c=\frac{1}{\sqrt{3} \pi^{2}}$. Now we can determine the combination which corresponds to the correct integral choice of the geometric period $\Pi^{(4)}$ as

$$
\Pi^{(4)}=2 c \nu+X^{0}
$$

from the uniquely defined periods $\left(X^{0}, \nu\right)$ at $z=0$ and $\Delta=0$. The analytic continuation of $\nu$ to $z=0$ fits nicely with our expectation from above and fixes most of the numerical coefficients in (5.27) universally

$$
a_{0}=\frac{\zeta(4)}{2^{4}(2 \pi i)^{6}} \int_{\tilde{X}_{4}} 5 c_{2}^{2},
$$

and

$a_{i}=-\frac{\zeta(3)}{(2 \pi i)^{3}} \int_{\tilde{X}_{4}} c_{3} \wedge J_{i}, \quad a_{i j}=\frac{\zeta(2)}{2(2 \pi i)^{2}} \int_{\tilde{X}_{4}} c_{2} \wedge J_{i} \wedge J_{j}, \quad a_{i j k}=\tilde{c} \int_{\tilde{X}_{4}} \imath_{*}\left(c_{1}\left(J_{i}\right)\right) \wedge J_{j} \wedge J_{k}$,

where as before $c_{i}=c_{i}\left(T_{\tilde{X}_{4}}\right)$ and $c_{1}\left(J_{i}\right)$ is the first Chern class of the divisor associated to $J_{i}$ which is mapped to a four-form via the Gysin homomorphism $\imath_{*}$ of the embedding map of this divisor into $\tilde{X}_{4}$. This is the generalization of (2.4) to the case of Calabi-Yau fourfolds. To be precise, the coefficients $a_{i j k}$ are not fixed by the sextic example, because it turns out to be zero in this case, and the canonical choice of $\Pi^{(4)}$ (5.34). This does not mean that it is absent in general. Rather it implies that it is physically irrelevant for the sextic because the divisibility of the correctly normalised solution, which is cubic in the logarithms, allows an integral symplectic choice of the periods in which this term can be set to zero. This might not be in general the case and other hypersurface in weighted projective space indicate that $\tilde{c}=1$. It is similarly possible to use the orbifold monodromy to fix the exact integral choice of the other periods. The principal form of the terms should again follow from the Frobenius method.

We conclude with some remarks about the enumerative geometry of the prepotentials $F^{0}(\gamma)$ of (5.23). As in the threefold case there is an enumerative geometry or counting

\footnotetext{
${ }^{17}$ The sign is chosen so that the $t^{4}$ term in $\Pi^{(4)}$ comes out with positive sign.
} 
interpretation of mirror symmetry in higher dimensions for the A-model [55]. The results and formulas necessary for our analysis are summarized in section 6 to which we refer at several points. As will be discussed there, the flux $\gamma$ is necessary to reduce to a counting of curves with the prepotential $F(\gamma)$ as a generating function, cf. (6.4). The prepotential furthermore has a $\mathrm{Li}_{2}$-structure and it is now possible to calculate the genus zero BPS invariants $n_{\beta}^{0}(\gamma)$ of section 6 for a suitable basis of $H_{V}^{2,2}\left(\tilde{X}_{4}\right)$ and $\beta$ in $H_{2}(\tilde{X}, \mathbb{Z})$.

\subsection{Application to elliptic fourfolds}

For the matching of the flux and brane superpotentials (2.1) from the perspective of Ftheory, we use the following strategy. We identify the periods of the threefold fiber $Y_{3}$ of $X_{4}$ among the fourfold periods. This implies a matching of all instanton numbers as well as the classical terms on the mirror $\tilde{Y}_{3}$. Furthermore we explicitly identify fourfold periods that reproduce the physics of branes on the local geometry of $\tilde{Y}_{3}$ discussed in section 4.1, namely all disk instantons calculated in [8]. This is equivalent to the statement that we calculate the superpotential (2.6) and the D7-brane superpotential (2.6) for a specific brane flux from the fourfold perspective of F-theory where the closed BPS states of the fourfold are encoded in $F^{0}(\gamma)$. We explicitly show that there is an element $\hat{\gamma} \in H^{2,2}(M)$ such that the enumerative geometry on the threefold mirror pair $\left(\tilde{Y}_{3}, Y_{3}\right)$ with and without Harvey-Lawson branes is reproduced. The results presented below are of further importance for the discussion of the F-theory/heterotic string duality in section 3.4, where the space $Y_{3}$ is promoted to the background geometry of the heterotic string.

Here we will discuss the geometry $X_{4}$ introduced in section 4.3 and refer to appendix B for further examples. The Calabi-Yau geometry at hand has four complex moduli. We find that the moduli dependence of the fourfold periods is determined by a complete set of six Picard-Fuchs operators which are linear differential operators $\mathcal{L}_{\alpha}, \alpha=1, \ldots, 6$ of order $(3,2,2,2,3,2)$, that can be obtained from the $\mathbb{C}^{*}$ symmetries of period integrals associated to the charge vectors $\ell_{I}^{(1)}, \ell_{I}^{(2)}, \ell_{I}^{(3)}, \ell_{I}^{(4)}, \ell_{I}^{(1)}+\ell_{I}^{(3)}, \ell_{I}^{(3)}+\ell_{I}^{(4)}$, by the methods described in [21. We use logarithmic derivatives $\theta_{a}=z_{a} \frac{d}{d z_{a}}$ in the canonical complex variables [21] and write down only the leading piece of the differential equations $\mathcal{L}_{\alpha}^{\lim }=\lim _{z_{a} \rightarrow 0} \mathcal{L}_{\alpha}\left(\theta_{a}, z_{a}\right)$, $a=1, \ldots, 4$. E.g. for the case at hand we have

$$
\begin{array}{llll}
\mathcal{L}_{1}^{\lim }=\theta_{1}^{2}\left(\theta_{3}-\theta_{1}-\theta_{4}\right), & \mathcal{L}_{2}^{\lim }=\theta_{2}\left(\theta_{2}-2 \theta_{1}-\theta_{3}-\theta_{4}\right), & \mathcal{L}_{3}^{\lim }=\left(\theta_{1}-\theta_{3}\right)\left(\theta_{3}-\theta_{4}\right), \\
\mathcal{L}_{4}^{\lim }=\theta_{4}\left(\theta_{1}-\theta_{3}+\theta_{4}\right), & \mathcal{L}_{5}^{\lim }=\theta_{1}^{2}\left(\theta_{4}-\theta_{3}\right), & \mathcal{L}_{6}^{\lim }=\theta_{4}\left(\theta_{1}-\theta_{3}\right) .
\end{array}
$$

For the complete Picard-Fuchs equations as well as the cohomology basis we extract from them we refer to appendix A.

Applying (5.17) it is easy to see that the there are $(1,4,6,4,1)$ generators of the ring 
$\mathcal{R}$ of degree $0, \ldots, 4$, which are

\begin{tabular}{|c|c|}
\hline $\mathcal{R}^{(0)}$ & 1 \\
\hline $\mathcal{R}_{a}^{(1)}$ & $\theta_{1}, \quad \theta_{2}, \quad \theta_{3}, \quad \theta_{4}$ \\
\hline $\mathcal{R}_{\alpha}^{(2)}$ & $\theta_{1}^{2}, \quad\left(\theta_{1}+\theta_{3}\right) \theta_{4}, \quad\left(\theta_{1}+\theta_{3}\right) \theta_{3}, \quad\left(\theta_{1}+2 \theta_{2}\right) \theta_{2}, \quad\left(\theta_{2}+\theta_{4}\right) \theta_{2}, \quad\left(\theta_{2}+\theta_{3}\right) \theta_{2}$ \\
\hline $\mathcal{R}_{a}^{(3)}$ & $\begin{array}{c}\left(\theta_{3}+\theta_{4}\right)\left(\theta_{1}^{2}+\theta_{1} \theta_{3}+\theta_{3}^{2}\right), \quad \theta_{2}\left(\theta_{3}^{2}+3 \theta_{2} \theta_{3}+5 \theta_{2}^{2}+\theta_{1}\left(\theta_{2}+\theta_{3}\right)\right) \\
\theta_{2}\left(\theta_{1}\left(\theta_{2}+\theta_{4}\right)+\theta_{4}\left(\theta_{3}+3 \theta_{2}\right)+\theta_{2}\left(\theta_{3}+6 \theta_{2}\right)\right), \quad \theta_{2}\left(\theta_{1}^{2}+2 \theta_{1} \theta_{2}+4 \theta_{2}^{2}\right)\end{array}$ \\
\hline $\mathcal{R}^{(4)}$ & $\begin{array}{c}\theta_{4}\left(\theta_{1}^{2} \theta_{2}+3 \theta_{1} \theta_{2}^{2}+9 \theta_{2}^{3}+\theta_{1} \theta_{2} \theta_{3}+3 \theta_{2}^{2} \theta_{3}+\theta_{2} \theta_{3}^{2}\right) \\
+\theta_{2}\left(46 \theta_{2}^{3}+15 \theta_{2}^{2} \theta_{3}+4 \theta_{2} \theta_{3}^{2}+\theta_{3}^{3}+\theta_{1}^{2}\left(2 \theta_{2}+\theta_{3}\right)+\theta_{1}\left(11 \theta_{2}^{2}+4 \theta_{2} \theta_{3}+\theta_{3}^{2}\right)\right)\end{array}$ \\
\hline
\end{tabular}

These can be associated to solutions of the Picard-Fuchs equations and to a choice of basis elements of the Chow ring as explained in section 5.3. At grade $k=2$ the leading solutions $\mathbb{L}^{(k) \alpha}$ of the Picard-Fuchs system (A.3) which are normalized to obey $\mathcal{R}_{\alpha}^{(k)} \mathbb{L}^{(k) \beta}=\delta_{\alpha}^{\beta}$ are then given by

$$
\begin{gathered}
\mathbb{L}^{(2) 1}=l_{1}^{2}, \quad \mathbb{L}^{(2) 2}=\frac{1}{2} l_{4}\left(l_{1}+l_{3}\right), \quad \mathbb{L}^{(2) 3}=\frac{1}{2} l_{3}\left(l_{1}+l_{3}\right), \\
\mathbb{L}^{(2) 4}=\frac{1}{7} l_{2}\left(3 l_{1}-2\left(l_{3}+l_{4}-l_{2}\right)\right), \quad \mathbb{L}^{(2) 5}=\frac{1}{7} l_{2}\left(-2 l_{1}+l_{2}+6 l_{4}-l_{3}\right), \\
\mathbb{L}^{(2) 6}=\frac{1}{7} l_{2}\left(-2 l_{1}+l_{2}+6 l_{3}-l_{4}\right),
\end{gathered}
$$

where we used the abbreviation $\log \left(z_{k}\right) \equiv l_{k}$ and omitted the prefactor $X_{0}$. In comparison to the complete solutions $\Pi^{(2) \alpha}$ of the Picard-Fuchs equations we omitted terms of order $\mathcal{O}(l)$ as in (5.18) and (5.19). Since we are calculating the holomorphic potentials $F(\gamma)$ of (5.23) and the corresponding BPS-invariants we have to change the basis of solutions such that to any operator $\mathcal{R}_{\alpha}^{(2)}$ in (5.38) we associate a solution with leading logarithm determined by the classical intersection $C_{a b \alpha}^{0(1,1,2)}$ of (5.13),

$$
\mathbb{L}_{\alpha}^{(2)}=\frac{1}{2} X_{0} C_{\alpha a b}^{0} l_{a} l_{b}
$$

From the above classical intersection data in $\mathcal{R}^{(4)}$ we obtain the leading terms $\mathbb{L}_{\alpha}^{(2)}$ which are related to the leading periods $\mathbb{L}^{(2) \alpha}$ of the four-form $\Omega$ in $(5.39)$ by $\mathbb{L}_{\alpha}^{(2)}=\mathbb{L}^{(2) \beta} \eta_{\alpha \beta}^{(2)}$.

As in the discussion after eq. (5.21) the choice of periods $\Pi^{(2) \alpha}$ with leading terms $\mathbb{L}^{(2) \alpha}$ corresponds to a particular choice of a basis $\hat{\gamma}_{\alpha}^{(2)}$ of $H_{V}^{2,2}(\tilde{X})$. In fact, by construction one finds

$$
\hat{\gamma}_{\alpha}^{(2)}=\left.\mathcal{R}_{\alpha}^{(k)} \Omega\right|_{z=0}
$$

However, this choice of basis for $H_{V}^{2,2}(\tilde{X})$ is not necessarily a basis of integral cohomology. An integral basis can, however, be determined by an appropriate basis change. We first 
note that the Kähler generator $J_{4}$ can be identified as the class of the Calabi-Yau threefold fiber $\tilde{Y}_{3}$ (see appendix $\mathrm{A}$ for more details on this identification). Moreover, one finds the identification of the fourfold Kähler generators $J_{i}$ with the threefold generators $J_{k}\left(\tilde{Y}_{3}\right)$ as

$$
J_{1}+J_{3} \leftrightarrow J_{1}\left(\tilde{Y}_{3}\right), \quad J_{2} \leftrightarrow J_{2}\left(\tilde{Y}_{3}\right)
$$

by comparing the coefficient of $J_{4}$ in the intersection form $\mathcal{C}_{0}\left(\tilde{X}_{4}\right)$, given in (A.2), with the threefold intersections $\mathcal{C}_{0}\left(\tilde{Y}_{3}\right)$ in (4.6). A subset of the basis elements of the fourfold integral basis are now naturally induced from the threefold integral basis. In order to do this one identifies the threefold periods $\partial_{i} F_{\tilde{Y}_{3}}$, with derivatives in the directions $J_{1}\left(\tilde{Y}_{3}\right)$ and $J_{2}\left(\tilde{Y}_{3}\right)$, with an appropriate linear combination of the fourfold periods $\Pi^{(2) \alpha}$ [38]. In other words one determines a new basis $\hat{\gamma}_{i}^{(2)}$ such that

$$
\partial_{i} F_{\tilde{Y}_{3}}=\left.\left.F^{0}\left(\hat{\gamma}_{i}^{(2)}\right)\right|_{z_{4}=0} \equiv \Pi_{i}^{(2)}\right|_{z_{4}=0} .
$$

In this matching both the classical part of the periods as well as the threefold BPS invariants $n_{d_{1}, d_{2}}$ and fourfold BPS invariants $n_{d_{1}, d_{2}, d_{1}, 0}(\gamma)$ have to match in the large $\mathrm{P}^{1}$-base limit.

The match (5.43) is most easily performed by first comparing the classical parts of the periods. In fact, using the classical intersections of $\tilde{Y}_{3}$ in (4.6) one deduces that the leading parts of the threefold periods are

$$
\mathbb{L}_{1}\left(Y_{3}\right)=\frac{1}{2} X_{0} \tilde{l}_{2}\left(2 \tilde{l}_{1}+3 \tilde{l}_{2}\right) \quad \mathbb{L}_{2}\left(Y_{3}\right)=\frac{1}{2} X_{0}\left(\tilde{l}_{1}+3 \tilde{l}_{2}\right)^{2}
$$

where $\tilde{l}_{i}=\log \tilde{z}_{i}$ correspond to the two threefold directions $J_{k}\left(\tilde{Y}_{3}\right)$ in (5.42). Using (5.42) and (5.43) one then finds the appropriately normalized leading fourfold periods

$$
\mathbb{L}_{2}^{(2)}=\frac{1}{2} X_{0} l_{2}\left(2 l_{1}+3 l_{2}+2 l_{3}\right) \quad \mathbb{L}_{5}^{(2)}=\frac{1}{2} X_{0}\left(l_{1}+3 l_{2}+l_{3}\right)^{2} .
$$

A direct computation also shows that the threefold BPS invariants $d_{i} n_{d_{1}, d_{2}}$ and fourfold BPS invariants $n_{d_{1}, d_{2}, d_{1}, 0}\left(\hat{\gamma}_{i}\right)$ match in the large $\mathrm{P}^{1}$-base limit, such that (5.43) is established on the classical as well as quantum level. This match fixes corresponding integral basis elements of $H_{V}^{2,2}\left(\tilde{X}_{4}\right)$ as follows. First we determine those two ring elements $\tilde{\mathcal{R}}_{\alpha}^{(2)}$, $\alpha=2,5$, such that we obtain (5.45) from them using (5.40) . We complete them to a new basis of ring elements $\tilde{\mathcal{R}}_{\alpha}^{(2)}$ by choosing

$$
\begin{gathered}
\tilde{\mathcal{R}}_{1}^{(2)}=\theta_{1}^{2}, \quad \tilde{\mathcal{R}}_{2}^{(2)}=\frac{1}{2} \theta_{4}\left(\theta_{1}+\theta_{3}\right), \quad \tilde{\mathcal{R}}_{3}^{(2)}=\frac{1}{2} \theta_{3}\left(\theta_{1}+\theta_{3}\right), \\
\tilde{\mathcal{R}}_{4}^{(2)}=\frac{1}{7} \theta_{2}\left(3 \theta_{1}-2\left(\theta_{3}+\theta_{4}-\theta_{2}\right)\right), \quad \tilde{\mathcal{R}}_{5}^{(2)}=\frac{1}{7} \theta_{2}\left(-2 \theta_{1}+\theta_{2}+6 \theta_{4}-\theta_{3}\right), \\
\tilde{\mathcal{R}}_{6}^{(2)}=\frac{1}{7} \theta_{2}\left(-2 \theta_{1}+\theta_{2}+6 \theta_{3}-\theta_{4}\right) .
\end{gathered}
$$

These operators fix the two integral basis elements

$$
\hat{\gamma}_{2}^{(2)}=\left.\tilde{\mathcal{R}}_{2}^{(2)} \Omega\right|_{z=0}, \quad \hat{\gamma}_{5}^{(2)}=\left.\tilde{\mathcal{R}}_{5}^{(2)} \Omega\right|_{z=0} .
$$


which reproduce the corresponding part of the flux superpotential (2.5) on $Y_{3}$ for $\hat{N}_{i}=0$ when turning on four-form flux on $X_{4}$ in these directions,

$$
W_{\text {flux }} \equiv M^{1} F^{0}\left(\hat{\gamma}_{2}^{(2)}\right)+M^{2} F^{0}\left(\hat{\gamma}_{5}^{(2)}\right)=\int_{X_{4}} \Omega \wedge G_{4}=M^{1} \Pi_{2}^{(2)}+M^{2} \Pi_{5}^{(2)}
$$

for the $G_{4}$-flux choice

$$
G_{4}=M^{1} \hat{\gamma}_{2}^{(2)}+M^{2} \hat{\gamma}_{5}^{(2)}
$$

For the choices $M^{i}=1$ we extract the invariants $d_{i} n_{d_{1}, d_{2}}$ from this superpotential, i.e. from the prepotentials $F^{0}\left(\hat{\gamma}_{2}^{(2)}\right)$ and $F^{0}\left(\hat{\gamma}_{5}^{(2)}\right)$. We note that the above grade $k=2$ basis elements (5.46) become under $\theta_{i} \leftrightarrow l_{i}$ exactly the leading solutions of the PicardFuchs system (5.39). Using the same identification we find $\mathbb{L}^{(2) 2}=X_{0}\left(l_{1}+l_{3}\right) l_{4}$ and $\mathbb{L}^{(2) 5}=X_{0}\left(l_{2}+l_{4}\right) l_{2}$ as the leading behaviour of corresponding periods $\int_{\gamma_{\alpha}^{2}} \Omega=\Pi^{(2) \alpha}$ of the holomorphic four-form. This agrees with the naive expectation from the large base limit that a partial factorization of the periods occurs as $t_{4} \cdot t_{i}^{\tilde{Y}_{3}}$ for $t_{i}^{\tilde{Y}_{3}}, i=1,2$, the two classes in $\tilde{Y}_{3}[38]$.

It is one crucial point of our whole analysis that we can extend this matching of threefold invariants even for disk invariants counting curves with boundaries on Lagrangian cycles $L$ in $\tilde{Y}_{3}$. Having explained the F-theory origin of this fact before we will here explicitly find the flux choice in $H_{H}^{(2,2)}\left(X_{4}\right)$ for which the flux superpotential (3.4) on the fourfold reproduces the brane superpotential (2.6). By construction our fourfold $\tilde{X}_{4}$ inherits information of the fiber $\tilde{Y}_{3}$ and in particular the local limit geometry $\mathcal{O}(-3) \rightarrow \mathbb{P}_{2}$ for which the disk invariants have been computed in [8]. As noted earlier the brane data is translated to the F-theory picture of the fourfold $\tilde{X}_{4}$ by the Mori cone generator $\ell^{(3)}$ and its dual divisor $J_{3}$. Therefore, we expect to reproduce all classical terms as well as extract the disk instantons of [8] from the Gromov-Witten invariants $n_{d, 0, d+k, 0}\left(\hat{\gamma}_{3}\right)$ of a period that we construct via (5.40) from operators of (5.39) of the form $\mathcal{R}_{\gamma}^{(2)}=\theta_{3}\left(\theta_{1}+\theta_{3}\right)+\ldots$. However, the geometry is more complicated and the ring element $\mathcal{R}_{\gamma}^{(2)}$ with this property is not unique. It takes the form

$$
\mathcal{R}_{\gamma}^{(2)}=-\mathcal{R}_{1}^{(2)}+\frac{1}{3} \mathcal{R}_{2}^{(2)}+\mathcal{R}_{3}^{(2)}=-\theta_{1}^{2}+\frac{1}{2} \theta_{3}\left(\theta_{1}+\theta_{3}\right)+\frac{1}{6} \theta_{4}\left(\theta_{1}+\theta_{3}\right)
$$

that is the most convenient choice by setting the arbitrary coefficients of $\mathcal{R}_{\alpha}^{(2)}, \alpha=4,5,6$, to zero. We note that only the coefficient in front of $\mathcal{R}_{3}^{(2)}$ was fixed to unity by the requirement of reproducing the disk instanton invariants. The two further coefficients $\left(-1, \frac{1}{3}\right)$ were fixed by the requirement of reproducing the Gromov-Witten invariants $n_{d}$ of local $\mathbb{P}^{2}$ [56] by the fourfold invariants $n_{d, 0, d, 0} \equiv n_{d}$, i.e. for $m=0$, as explained below. The relation between $\mathcal{R}_{\gamma}^{(2)}$ and the corresponding solution is via $\hat{\gamma}=\left.\mathcal{R}_{\gamma} \Omega\right|_{z=0}$ and $\Pi^{(2) \gamma}=\int_{\gamma} \Omega$, i.e. $\mathcal{R}_{\gamma}^{(2)} \Pi^{(2) \gamma}=1$, so that

$$
\mathbb{L}^{(2) \gamma}=-X_{0} l_{1}^{2}, \quad \mathbb{L}_{\gamma}^{(2)}=\frac{1}{6} X_{0} l_{2}\left(8 l_{1}+9 l_{2}+2 l_{3}\right)
$$


This implies that we have explicitly calculated the D7-brane superpotential (2.6) from the fourfold superpotential (3.4) by turning on the flux $G_{4}=\hat{\gamma}$,

$$
W_{\mathrm{D} 7} \equiv F^{0}(\hat{\gamma})=\int \Omega \wedge \hat{\gamma}=\Pi_{\gamma}^{(2)}
$$

If we list the numbers $n_{d_{1}, 0, d_{3}, 0}(\hat{\gamma})$ extracted from $F^{0}(\hat{\gamma})$ we get the following table. The BPS invariants of the holomorphic disks depend only on the relative homology class

\begin{tabular}{|c|rrrrrrr|}
\hline $\mathrm{d}_{1}$ & $d_{3}=0$ & $d_{3}=1$ & $d_{3}=2$ & $d_{3}=3$ & $d_{3}=4$ & $d_{3}=5$ & $d_{3}=6$ \\
\hline 0 & 0 & 1 & 0 & 0 & 0 & 0 & 0 \\
1 & 1 & $n_{1}$ & -1 & -1 & -1 & -1 & -1 \\
2 & -1 & -2 & $2 n_{2}$ & 5 & 7 & 9 & 12 \\
3 & 1 & 4 & 12 & $3 n_{3}$ & -40 & -61 & -93 \\
4 & -2 & -10 & -32 & -104 & $4 n_{4}$ & 399 & 648 \\
5 & 5 & 28 & 102 & 326 & 1085 & $5 n_{5}$ & -4524 \\
6 & -13 & -84 & -344 & -1160 & -3708 & -12660 & $6 n_{6}$ \\
\hline
\end{tabular}

Table 5.2: BPS invariants $n_{d_{1}, 0, d_{3}, 0}(\hat{\gamma})$ for the disks. With the identification $d_{3}-d_{1}=m$ (winding) and $d_{1}=d\left(\mathbb{P}^{2}\right.$ degree $)$ this agrees with Tab. 5 in $[8]$.

of the latter. In the table $d_{3}-d_{1}=m$ labels the winding number of the disks and $d_{1}=d$ the degree with respect to canonical class of $\mathrm{P}^{2}$, i.e. if the open string disk superpotential is in terms of the closed string parameter $q=e^{2 \pi i t}$ and the open string string parameter $Q=e^{2 \pi i \hat{t}}$ for the outer brane defined as

$$
W=a_{t t} t^{2}+a_{t \hat{t}} t \hat{t}+a_{\hat{t} \hat{t}} \hat{t}^{2}+a_{t} t+a_{\hat{t}} \hat{t}+a_{0}+\sum_{d=1}^{\infty} \sum_{m=-d}^{\infty} n_{d, m} \operatorname{Li}_{2}\left(q^{d} Q^{m}\right)
$$

then $n_{d_{1}, 0, d_{3}, 0}=n_{d_{1}, d_{3}-d_{1}}$. Note that the numbers $n_{d_{1}, 0}$ are not calculated in the framework of [8]. However it is natural and calculable in the topological vertex formalism that they should be identified with $d n_{d}$, where $n_{d}$ is the closed string genus zero BPS invariant, defined via the prepotential as $F=\sum_{d=1}^{\infty} n_{d} \operatorname{Li}_{3}\left(q^{d}\right)$. The factor of $d$ comes from the fact that we identify $W=\frac{d}{d t} F$. This interpretation as $n_{d, 0, d, 0}=d n_{d}$ could be consistently imposed and yields two further conditions as mentioned above.

To obtain the open BPS invariants of phase III of [8], we use the phase II of (4.22). In this phase the fiber class is not realized as a generator of the Kähler cone. However we readily recover the classes of $\tilde{Y}_{3}$ as

$$
J_{1} \leftrightarrow J_{1}\left(\tilde{Y}_{3}\right) \quad J_{2}+J_{3} \leftrightarrow J_{2}\left(\tilde{Y}_{3}\right)
$$


by comparison of the Mori cone (4.22) with the Mori cone (4.5) of $\tilde{Y}_{3}$. Then we fix a basis $\mathcal{R}_{\alpha}^{(2)}$ of the ring at grade two as

$$
\theta_{1}^{2}, \quad 2 \theta_{2}\left(\theta_{1}+3 \theta_{3}\right), \quad \theta_{3}\left(\theta_{1}+3 \theta_{3}\right), \quad \theta_{1} \theta_{4}, \quad \theta_{2}^{2}, \quad\left(\theta_{2}+\theta_{3}\right)\left(2 \theta_{3}+\theta_{4}\right),
$$

from which we obtain a basis of dual solutions $\mathbb{L}^{(k) \alpha}$ to the Picard-Fuchs system (A.9)

$$
\begin{gathered}
\mathbb{L}^{(2) 1}=l_{1}^{2}, \quad \mathbb{L}^{(2) 2}=\frac{1}{140}\left(l_{1}\left(16 l_{2}+9 l_{3}\right)+3\left(l_{2}\left(6 l_{3}-5 l_{4}\right)-l_{3}\left(l_{3}+5 l_{4}\right)\right)\right), \\
\mathbb{L}^{(2) 3}=\frac{1}{70}\left(l_{1}\left(9 l_{2}+16 l_{3}\right)-3\left(l_{3}\left(-6 l_{3}+5 l_{4}\right)+l_{2}\left(l_{3}+5 l_{4}\right)\right)\right), \quad \mathbb{L}^{(2) 4}=l_{1} l_{4}, \\
\mathbb{L}^{(2) 5}=l_{2}^{2}, \quad \mathbb{L}^{(2) 6}=\frac{1}{14}\left(l_{2}+l_{3}\right)\left(-3 l_{1}+l_{3}+5 l_{4}\right) .
\end{gathered}
$$

Next we construct two solutions with leading logarithms matching the two threefold periods of (5.44) for which we are able to match the threefold invariants $d_{i} n_{d_{1}, d_{2}}$ in the large base limit as well. The leading logarithms of these fourfold periods read

$$
\begin{aligned}
& \mathbb{L}_{4}^{(2)}=\frac{1}{2} X_{0}\left(l_{1}+3\left(l_{2}+l_{3}\right)\right)^{2}, \\
& \mathbb{L}_{6}^{(2)}=\frac{1}{2} X_{0}\left(l_{2}+l_{3}\right)\left(2 l_{1}+3\left(l_{2}+l_{3}\right)\right) .
\end{aligned}
$$

which is in perfect agreement with (5.44) under the identification (5.54). We fix the corresponding operators $\tilde{\mathcal{R}}_{4}^{(2)}, \tilde{\mathcal{R}}_{6}^{(2)}$ by matching the above two leading logarithms by the classical intersections $C_{\alpha a b}^{0}$ via (5.40). We complete them to a basis of $\tilde{\mathcal{R}}^{(2)}$ as follows

$$
\begin{gathered}
\tilde{\mathcal{R}}_{1}^{(2)}=\theta_{1}^{2}, \quad \tilde{\mathcal{R}}_{2}^{(2)}=\frac{1}{140}\left(\theta_{1}\left(16 \theta_{2}+9 \theta_{3}\right)+3\left(\theta_{2}\left(6 \theta_{3}-5 \theta_{4}\right)-\theta_{3}\left(\theta_{3}+5 \theta_{4}\right)\right)\right), \\
\tilde{\mathcal{R}}_{3}^{(2)}=\frac{1}{70}\left(\theta_{1}\left(9 \theta_{2}+16 \theta_{3}\right)-3\left(\theta_{3}\left(-6 \theta_{3}+5 \theta_{4}\right)+\theta_{2}\left(\theta_{3}+5 \theta_{4}\right)\right)\right), \quad \tilde{\mathcal{R}}_{4}^{(2)}=\theta_{1} \theta_{4}, \\
\tilde{\mathcal{R}}_{5}^{(2)}=\theta_{2}^{2}, \quad \tilde{\mathcal{R}}_{6}^{(2)}=\frac{1}{14}\left(\theta_{2}+\theta_{3}\right)\left(-3 \theta_{1}+\theta_{3}+5 \theta_{4}\right),
\end{gathered}
$$

where again this basis relates to the leading periods (5.56) by $\theta_{i} \leftrightarrow l_{i}$. The corresponding integral basis elements of $H_{H}^{2,2}\left(X_{4}\right)$ read

$$
\hat{\gamma}_{4}^{(2)}=\left.\tilde{\mathcal{R}}_{4}^{(2)} \Omega\right|_{z=0}, \quad \hat{\gamma}_{6}^{(2)}=\left.\tilde{\mathcal{R}}_{6}^{(2)} \Omega\right|_{z=0} .
$$

Furthermore, we determine the ring element $\mathcal{R}_{\gamma}^{(2)}$ that matches the open superpotential by turning on four-form flux in the direction $\hat{\gamma}=\left.\mathcal{R}_{\gamma}^{(2)} \Omega\right|_{z=0}$. Again we fix

$$
\mathcal{R}_{\gamma}^{(2)}=a_{1} \mathcal{R}_{2}^{(2)}-\frac{1}{10}\left(1+6 a_{2}\right) \mathcal{R}_{3}^{(2)}+\mathcal{R}_{4}^{(2)}+a_{3} \mathcal{R}_{5}^{(2)}+a_{2} \mathcal{R}_{6}^{(2)}
$$

by extracting the disk invariants from the solution associated to it via (5.40) which reads

$$
\begin{aligned}
\mathbb{L}^{(2) \gamma} & =c\left(a_{1}\right) X_{0} l_{2}\left(l_{1}+3 l_{3}\right), \\
\mathbb{L}_{\gamma}^{(2)} & =\frac{1}{6}\left(l_{2}+l_{3}\right)\left(2 l_{1}+3\left(l_{2}+l_{3}\right)\right)-\frac{1}{10}\left(l_{1}+3\left(l_{2}+l_{3}\right)\right)\left(3 l_{1}+29 l_{2}+29 l_{3}+10 l_{4}\right) .
\end{aligned}
$$

Here we explicitly displayed the dependence on the three free parameters $a_{i}$ for $\mathbb{L}^{(2) \gamma}$ by $c\left(a_{1}\right)=\frac{7}{9+140 a_{1}}$ and evaluated $\mathbb{L}_{\gamma}^{(2)}$ for the convenient choice $a_{i}=0$. 


\begin{tabular}{|r|rrrrrrr|}
\hline $\mathrm{d}$ & $k=0$ & $k=1$ & $k=2$ & $k=3$ & $k=4$ & $k=5$ & $k=6$ \\
\hline 0 & 0 & $n_{1}$ & $2 n_{2}$ & $3 n_{3}$ & $4 n_{4}$ & $5 n_{5}$ & $6 n_{6}$ \\
1 & -1 & 2 & -5 & 32 & -286 & 3038 & -35870 \\
2 & 0 & 1 & -4 & 21 & -180 & 1885 & -21952 \\
3 & 0 & 1 & -3 & 18 & -153 & 1560 & -17910 \\
4 & 0 & 1 & -4 & 20 & -160 & 1595 & -17976 \\
5 & 0 & 1 & -5 & 26 & -196 & 1875 & -20644 \\
6 & 0 & 1 & -7 & 36 & -260 & 2403 & -25812 \\
\hline
\end{tabular}

Table 5.3: BPS invariants $n_{k, 0, i, 0}(\gamma)$ for the disks of the second triangulation.

\section{Basics of enumerative geometry}

In this section we want to describe from the A-model perspective the relevant enumerative quantities, which are calculated in this paper in the B-model using mirror symmetry. Important circumstantial evidence for the open-string/fourfold duality approach [13, 14] advocated in this paper is the identical integral structure of the generating functions.

However as we will review in the following, the absence of higher genus invariants on smooth fourfolds as opposed to the open string setting might be a hint that this duality discussed relies merely on an embedding of the open/closed moduli space into the closed moduli space as discussed, e.g. in [15], rather than on a full duality of physical theories. A possibility to avoid this conclusion would be that one has in general to consider singular fourfolds, typical for an F-theory compactification with degenerate elliptic fiber along the zero-locus of the discriminant.

\subsection{Closed GW invariants}

First we review the theory of closed Gromov-Witten invariants, i.e. the theory of holomorphic maps

$$
\phi: \Sigma_{g} \rightarrow \tilde{X}
$$

from an oriented closed curve $\Sigma_{g}$ into a Calabi-Yau manifold $\tilde{X}$. We do not consider marked points. It can be defined mathematically rigorously in general and explicitly calculated using localization techniques if $\tilde{X}$ is represented e.g. by a hypersurface in a toric variety. Here $g$ is the genus of the domain curve and we denote by $\beta \in H_{2}(\tilde{X}, \mathbb{Z})$ the homology class of the image curve. One measures the multi-degrees of the latter $\beta=\sum_{i=1}^{h^{1,1}} d_{i} \beta_{i}$ w.r.t. to an ample polarization $L$ of $\tilde{X}$, i.e. $\operatorname{deg}(\beta)=\int_{\beta} c_{1}(L)=\sum_{i=1}^{h^{1,1}} d_{i} t_{i}$ with $d_{i} \in \mathbb{N}_{+}$. In string theory and in the context of the mirror symmetry the volume 
of the curve $\beta_{i}$ is complexified by an integral over the antisymmetric two-form field $B$. Thus, one defines the complexified closed Kähler moduli $t_{i}=\int_{\beta_{i}}\left(B+i c_{1}(L)\right)$.

For smooth $\tilde{X}$ the virtual (complex) dimension of the moduli space of these maps are computed by an index theorem and reads

$$
\operatorname{vir} \operatorname{dim} \mathcal{M}_{g}(\tilde{X}, \beta)=\int_{\beta} c_{1}(\tilde{X})+(\operatorname{dim} \tilde{X}-3)(1-g) .
$$

In particular for Calabi-Yau fourfolds one obtains vir $\operatorname{dim} \mathcal{M}_{g}\left(\tilde{X}_{4}, \beta\right)=1-g$. Thus in order to define genus zero Gromov-Witten invariants one requires an incidence relation of the curve with $k=(\operatorname{dim}(\tilde{X})-3)$ surfaces to reduce the dimension to zero in order to arrive at a well-defined counting problem. For fourfolds one thus needs one incidence surface and we denote the dual cycle of the surface by $\gamma \in H^{2,2}\left(\tilde{X}_{4}\right)$. Note that for $\operatorname{dim} \tilde{X} \geq 4$ and $g \geq 2$ the dimension of the moduli space is negative and no holomorphic maps exist. The Calabi-Yau threefolds are critical in the sense that the dimension of the moduli space for all genera is zero. Thus, in general invariants associated to the maps are non-zero for all values of $g$.

We define a generating function for each genus $g$ Gromov-Witten invariant as follows:

$$
F^{g}\left(\gamma_{1}\right)=\sum_{\beta \in H_{2}(\tilde{X}, \mathbb{Z})} r_{\beta}^{g}\left(\gamma_{1}, \ldots, \gamma_{k}\right) q^{\beta}
$$

They are labelled by $g, \beta$ and for $\operatorname{dim} \tilde{X} \geq 4$ also by cycles $\gamma_{i}$ dual to the incidence surfaces. Here $q^{\beta}$ is a shorthand notation for $q^{\beta}=\prod_{i=1}^{h^{1,1}} e^{2 \pi i t_{i} d_{i}}$. We note that this is not just a formal power series 18 , but rather has finite region of convergence for large volumes of the curves $\beta_{i}$, i.e. for $\operatorname{Im}\left(t_{i}\right) \gg 0$. This puts a bound on the growth of the Gromov-Witten invariants $r_{\beta}^{g}\left(\gamma_{1}\right)$. The contributions of the maps is divided by their automorphism groups and the associated Gromov-Witten invariants $r_{\beta}^{g}\left(\gamma_{1}, \ldots, \gamma_{k}\right)$ are in general rational.

Although the discussion of (6.2) indicates that the Gromov-Witten theory on higher dimensional Calabi-Yau manifolds is less rich than in the threefold case, one has a remarkable integrality structure associated to the invariants. In particular at genus zero one can define integer invariants $n_{\beta}^{g}\left(\gamma_{1}, \ldots \gamma_{k}\right) \in \mathbb{Z}$ for arbitrary $\operatorname{dim}(\tilde{X})=k+3$ dimensional manifolds as

$$
F^{0}\left(\gamma_{1}, \ldots, \gamma_{k}\right)=\frac{1}{2} C_{a b \gamma_{1} \cdots \gamma_{k}}^{0(1,1, n-2)} t^{a} t^{b}+b_{a \gamma_{1} \cdots \gamma_{k}}^{0} t^{a}+a_{\gamma_{1} \cdots \gamma_{k}}^{0}+\sum_{\beta>0} n_{\beta}^{g}\left(\gamma_{1}, \ldots, \gamma_{k}\right) \operatorname{Li}_{3-k}\left(q^{\beta}\right)
$$

\footnotetext{
${ }^{18}$ This is important for the interpretation of such terms in the effective action. In fact, analyticity allows to define such terms beyond the large radius limit in terms of period integrals on the mirror geometry.
} 
where $\operatorname{Li}_{p}(q)=\sum_{d=1}^{\infty} \frac{q^{d}}{d^{p}}$ and $C_{a b \gamma_{1} \cdots \gamma_{k}}^{0(1,1, n-2)}$ are the classical triple intersections. For threefolds an analogous formula was found in [22] and the multicovering was explained in [57]. Note that $b_{a \gamma_{1} \cdots \gamma_{k}}^{0}, a_{\gamma_{1} \cdots \gamma_{k}}^{0}$ are irrelevant for the quantum cohomology, as the latter is defined by the second derivative of $F^{0}\left(\gamma_{1}, \ldots, \gamma_{k}\right)$.

Genus one Gromov-Witten invariants exist on Calabi-Yau manifold of all dimensions with the need of incidence conditions as discussed above. For fourfolds the authors of [55] define the following integrality condition

$$
\begin{aligned}
F^{1}= & \sum_{\beta>0} n_{\beta}^{1} \frac{\sigma(d)}{d} q^{d \beta} \\
& +\frac{1}{24} \sum_{\beta>0} n_{\beta}^{0}\left(c_{2}(\tilde{X})\right) \log \left(1-q^{\beta}\right) \\
& -\frac{1}{24} \sum_{\beta_{1}, \beta_{2}} m_{\beta_{1}, \beta_{2}} \log \left(1-q^{\beta_{1}+\beta_{2}}\right) .
\end{aligned}
$$

Here the $m_{\beta_{1}, \beta_{2}}$ are so called meeting invariants, which are likewise integer as the $n_{\beta}^{g}(\cdot)$ and the function $\sigma$ is defined by $\sigma(d)=\sum_{i \mid d} i$.

Note for threefolds one also has the BPS state counting formula [58]

$$
F(\lambda, q)=\sum_{g=0}^{\infty} \lambda^{2 g-2} F^{g}=\sum_{\beta>0, g \geq 0, k>0} n_{\beta}^{g} \frac{1}{k}\left(2 \sin \frac{k \lambda}{2}\right)^{2 g-2} q^{k \beta}
$$

\subsection{Open GW invariants}

Let us come now to the open Gromov-Witten invariants on Calabi-Yau threefolds $\tilde{Y}$. They arise in the open topological A-model on $\tilde{Y}$. We consider a Calabi-Yau manifold $\tilde{Y}$ together with a special Lagrangian submanifold $L$ and consider a map from an oriented open Riemann surface, i.e. Riemann surface with boundary

$$
\psi: \Sigma_{g, h} \rightarrow(\tilde{Y}, L)
$$

into the Calabi-Yau manifold $\tilde{Y}$. Here the Riemann surface is mapped with a given winding number into $L$ such that the $h$ boundary circles $B_{i}$ of $\Sigma_{g, h}$ are mapped on nontrivial elements $\vec{\alpha}=\left(\alpha_{1}, \ldots, \alpha_{h}\right) \in H^{1}(L, \mathbb{Z})^{\oplus h}$. As in the closed case we do not consider marked points. For threefolds the moduli space $\mathcal{M}_{g, h}(\tilde{Y}, L, \beta, \vec{\alpha}, \mu)$ with the Maslov index $\mu$ has virtual dimension zero [59]. If $H^{1}(L, \mathbb{Z})$ is non-trivial, the special Lagrangian has a geometric deformation moduli. The open string moduli $\hat{t}_{i}$ are complexifications of the geometric moduli by the Wilson-Loop integrals of the flat $U(1)$ gauge connection on the brane. 
The open BPS state counting formula analogous to (6.6) was given in [60] and reads

$$
F(t, u)=\sum_{g=0, h=1}^{\infty} \lambda^{2 g-2+h} F_{n_{1}, \ldots, n_{h}}^{g}(t) \operatorname{tr} U^{n_{1}} \cdots U^{n_{h}}=i \sum_{n=1}^{\infty} \frac{n_{\beta, \mathcal{R}}^{g}}{2 n \sin \left(\frac{n \lambda}{2}\right)} q_{\lambda}^{n g} q^{n \beta} \operatorname{Tr}_{\mathcal{R}} U^{n}
$$

In particular the disk amplitude, which gives rise to the superpotential, is given by

$$
W=F_{h=1}^{0}=\sum_{\beta, m} \operatorname{Li}_{2}\left(q^{\beta} Q^{m}\right)
$$

with $Q=e^{2 \pi i \hat{t}}$. Comparison with (6.4) suggest that the counting problem of specific disks amplitudes can be mapped to the counting of rational curves in fourfolds since the integrality structure is the same and given by the $\mathrm{Li}_{2}$-structure.

\section{Conclusions}

In this work we have studied the holomorphic flux superpotential in F-theory compactifications on Calabi-Yau fourfolds. In F-theory the complex structure moduli of the elliptically fibered fourfold contain both the closed string and seven-brane moduli of the associated IIB theory. Thus, a non-trivial $G_{4}$-flux induces a superpotential for both closed and open moduli of the Type IIB background. We made use of this unified description of open and closed moduli to explicitly present the splitting of the fourfold complex structure moduli into threefold complex structure and brane moduli for a given example. This identification is further confirmed by heterotic/F-theory duality where we consistently matched F-theory and heterotic moduli. In particular, we used this to physically argue that the periods of the threefold $Y_{3}$ have to be contained in the periods of $X_{4}$, since $Y_{3}$ arises as the compactification space of the heterotic string by duality. Furthermore, we recovered the flux superpotential of the Type IIB string and the open-closed superpotential on the seven-brane with gauge flux inducing a five-brane charge from the fourfold perspective and comment on its heterotic interpretation.

The presence of a non-trivial superpotential for the complex structure moduli of the fourfold $X_{4}$ is of crucial importance in the study of F-theory vacua. In particular, as initiated in refs. [61, 62] , F-theory provides a promising framework to model supersymmetric GUT models with a remarkably realistic phenomenology on non-compact Calabi-Yau fourfolds. In such non-compact scenarios one can tune the complex structure moduli by hand to obtain realistic settings. However, eventually one has to compactify these models as recently done in [63, 64. This yields a large set of dynamical complex structure moduli and only a detained study of the F-theory flux superpotential will show whether phenomenologically preferred settings can indeed be stabilized by fluxes. The 
compact setups of ref. [63] are realized on explicit Calabi-Yau fourfolds in a toric ambient space, and it would be interesting to analyze the superpotential for a relevant subset of the complex structure moduli by the guideline presented in this work.

Let us summarize the methods applied in this work. Since the whole analysis of this work is based on the extensive use of mirror symmetry, we presented an account of basic facts and methods of closed and open mirror symmetry in section 2, After discussing the general structure of flux and D7-brane superpotentials in Calabi-Yau orientifolds we introduced the necessary technical machinery to construct Calabi-Yau hypersurfaces in compact toric Fano varieties. For open mirror symmetry we recalled the non-compact setting with Harvey-Lawson type branes dual to D5-branes which was crucial for our later re-interpretation of the open Gromov-Witten invariants as invariants originating from a seven-brane in the B-model geometry. In our F-theory analysis of section 4 the whole Riemann surface of the local mirror geometry was contained in the discriminant locus of the elliptic fibration that encodes seven-branes in F-theory. The open superpotential was induced on this seven-brane since a world-volume gauge flux induced five-brane charge.

Before delving into the details of these calculations we briefly introduced in section 3 the general concepts of F-theory and the toric construction of elliptic fourfolds with particular fibration structures encoding the non-perturbative physics of seven-branes as well as heterotic dual geometries. Here we put special emphasis on the flux superpotential and the supersymmetric F-theory fluxes. We completed our picture by briefly mentioning the concepts of the spectral cover construction for heterotic/F-theory duality, that we later on applied to identify moduli on both sides. After this preparation we started to embed the local A-model geometries with Harvey-Lawson type branes into compact threefolds in section 4. For the sake of clarity we chose as an explicit example local $\mathbb{P}^{2}$. We described the geometry of the elliptically fibered threefold $\tilde{Y}_{3}$ and its mirror $Y_{3}$ which is also elliptically fibered. We put strong emphasis on the Weierstrass model of $Y_{3}$ and checked that in the limit of large elliptic fiber, we obtained the local B-model geometry, a conic over an elliptic curve. Next, we gave a construction to associate a compact fourfold $\tilde{X}_{4}$, i.e. a five-dimensional reflexive polyhedron to the compact Calabi-Yau threefold $\tilde{Y}_{3}$. The fourfold $\tilde{X}_{4}$ contained $\tilde{Y}_{3}$ as generic fiber with base $\mathbb{P}^{1}$. There is a rich fibration structure on $\tilde{X}_{4}$ and we showed that the mirror geometry $X_{4}$ which was relevant for our study is also elliptically fibered such that F-theory is well-defined. We studied the geometry of $X_{4}$ in great detail and showed that the discriminant locus of $X_{4}$ contains a component which corresponds to the seven-brane in F-theory. Furthermore, both $X_{4}$ and its mirror are fibered by an elliptic $K 3$ which allows for a heterotic dual compactified on the elliptic threefold $Y_{3}$. For both the F-theory and the heterotic side we could determine the splitting of the complex structure moduli of $X_{4}$ into complex structure moduli and brane respectively spectral cover moduli. 
To actually compute the superpotential on fourfolds and to identify the threefold periods, we described mirror symmetry on Calabi-Yau fourfolds in more detail and also more formal aspects related to the topological A- and B-model defined on fourfolds. We took also a closer look on the properties of the periods of the fourfolds since the flux superpotential could be expressed as a linear sum of them. Here we put special emphasis on the determination of the integral base of $H_{V}^{(2,2)}\left(\tilde{X}_{4}\right)$. This made necessary to determine the classical terms in the maximally logarithmic period (5.27) as well as to perform the analytic continuation and monodromy analysis for the periods of sextic hypersurfaces. We used the identification of prepotentials (5.43) implying a matching of classical terms as well as instanton numbers of the corresponding periods to match the periods of $Y_{3}$. This way we determined a $G_{4}$-flux to match the flux superpotential in the form of (5.48). A similar analysis allowed for a $G_{4}$-flux matching the brane superpotential in (5.52). Thus we have explicitly checked, on the level of computing the superpotential and identification of periods, that the complex structure moduli space or the flux superpotential of F-theory contains the closed/open moduli space of the Type IIB theory with seven-branes. Equivalent statements could of course be inferred for the moduli space of the heterotic dual theory.

We concluded with section 6 where we provided a basic background in enumerative geometry and introduced some of the notions we used throughout our work. Further examples of fourfolds with more moduli were discussed in two appendices.

For possible future directions it would be interesting to analyze the structure of periods on fourfolds at other points of the moduli space including a general analysis of monodromies. Physical interpretations of their different structure compared to the threefold case might shed some light on additional massless states tightly related to singularities in the moduli space. Furthermore it might repay further studies to deduce the classical terms of the maximally logarithmic period from first principles. From a physical point of view a more thorough analysis on the other $G_{4}$-flux choices not considered in our work would be useful. In particular we note that the F-theory superpotential intrinsically contains non-perturbative corrections due to a non-trivial dilaton dependence of the fourfold periods that treat it on an equal footing to ordinary complex structure moduli.

\section{Acknowledgements}

We gratefully acknowledge discussions with Ralph Blumenhagen, Babak Haghighat, Hans Jockers, Wolfgang Lerche, Adrian Mertens, Marco Rauch, Johannes Walcher and Timo Weigand. TG would like to thank the KITP Santa Barbara, the MPI Munich and the Simons Center for Geometry and Physics, Stony Brook, for hospitality and support. This 
work was supported in parts by the European Union 6th framework program MRTN-CT2004-503069 "Quest for unification", MRTN-CT-2004-005104 "ForcesUniverse", MRTNCT-2006-035863 "UniverseNet", SFB-Transregio 33 "The Dark Universe" by the DFG. The work of T.-W.H. and D.K. is supported by the German Excellence Initiative via the graduate school "Bonn Cologne Graduate School". The work of D.K. is supported by a scholarship of the "Deutsche Telekom Stiftung".

\section{A Further topological data of the main example}

Here we supply the topological data of the fourfold $\tilde{X}_{4}$ that was omitted in the main text for convenience. Besides the intersection rings we will also present the full Picard-Fuchs system at the large radius/large complex structure point. These determine as explained in section 5 the primary vertical subspace $H_{V}^{p, p}\left(\tilde{X}_{4}\right)$ of the A-model.

As was mentioned before there are four triangulations whereas only three yield nonsingular varieties. Again we restrict our exposition to the two triangulations mentioned in section 4.3. For the following we label the points in the polyhedron $\Delta_{5}^{\tilde{X}}$ given in (4.22) consecutively by $\nu_{i}, i=0, \ldots, 9$ and associated coordinates $x_{i}$ to each $\nu_{i}$. Then the toric divisors are given by $D_{i}:=\left\{x_{i}=0\right\}$.

Phase I: In phase I of the toric variety defined by the polyhedron $\Delta_{5}^{\tilde{X}}$ in (4.22) one has the following Stanley-Reisner ideal

$$
S R=\left\{D_{3} D_{8}, D_{7} D_{9}, D_{8} D_{9}, D_{1} D_{5} D_{6}, D_{2} D_{3} D_{4}, D_{2} D_{4} D_{7}\right\}
$$

From this we compute by standard methods of toric geometry the intersection numbers

$$
\begin{aligned}
\mathcal{C}_{0}= & J_{4}\left(J_{1}^{2} J_{2}+J_{1} J_{3} J_{2}+J_{3}^{2} J_{2}+3 J_{1} J_{2}^{2}+3 J_{3} J_{2}^{2}+9 J_{2}^{3}\right)+J_{1}^{2} J_{3} J_{2}+J_{1} J_{3}^{2} J_{2}+J_{3}^{3} J_{2} \\
& +2 J_{1}^{2} J_{2}^{2}+4 J_{1} J_{3} J_{2}^{2}+4 J_{3}^{2} J_{2}^{2}+11 J_{1} J_{2}^{3}+15 J_{3} J_{2}^{3}+46 J_{2}^{4}, \\
\mathcal{C}_{2}= & 24 J_{1}^{2}+36 J_{1} J_{4}+48 J_{1} J_{3}+36 J_{4} J_{3}+48 J_{3}^{2}+128 J_{1} J_{2}+102 J_{2} J_{4} \\
& +172 J_{2} J_{3}+530 J_{2}^{2} \\
\mathcal{C}_{3}= & -660 J_{1}-540 J_{4}-900 J_{3}-2776 J_{2} .
\end{aligned}
$$

Here we denoted generators of the Kähler cone of (4.24) dual to the Mori cone by $J_{i}$ as before. The notation for the $\mathcal{C}_{k}$ is as follows. Denoting the dual two-forms to $J_{i}$ by $\omega_{i}$ the coefficients of the top intersection ring $\mathcal{C}_{0}$ are the quartic intersection numbers $J_{i} \cap J_{j} \cap J_{k} \cap J_{l}=\int_{\tilde{X}_{4}} \omega_{i} \wedge \omega_{j} \wedge \omega_{k} \wedge \omega_{l}$, while the coefficients of $\mathcal{C}_{2}$ and $\mathcal{C}_{3}$ are $\left[c_{2}\left(\tilde{X}_{4}\right)\right] \cap J_{i} \cap J_{j}=$ $\int_{\tilde{X}_{4}} c_{2} \wedge \omega_{i} \wedge \omega_{j}$ and $\left[c_{3}\left(\tilde{X}_{4}\right)\right] \cap J_{i}=\int_{\tilde{X}_{4}} c_{3} \wedge \omega_{i}$ respectively. 
As reviewed in section 5.3 the Picard-Fuchs operators of the mirror fourfold $X_{4}$ at the large complex structure point are calculated by the methods described in [21]. In the appropriate coordinates $z_{i}$ defined by (4.9) and evaluated in (4.32) we obtain the full Picard-Fuchs system on $\tilde{X}_{4}$ given by

$$
\begin{aligned}
\mathcal{L}_{1}^{I}= & -\theta_{1}^{2}\left(\theta_{1}+\theta_{4}-\theta_{3}\right) \\
& -\left(-1+\theta_{1}-\theta_{3}\right)\left(-2+2 \theta_{1}+\theta_{4}+\theta_{3}-\theta_{2}\right)\left(-1+2 \theta_{1}+\theta_{4}+\theta_{3}-\theta_{2}\right) z_{1}, \\
\mathcal{L}_{2}^{I}= & \theta_{2}\left(-2 \theta_{1}-\theta_{4}-\theta_{3}+\theta_{2}\right)-12\left(-5+6 \theta_{2}\right)\left(-1+6 \theta_{2}\right) z_{2}, \\
\mathcal{L}_{3}^{I}= & \left(\theta_{1}-\theta_{3}\right)\left(-\theta_{4}+\theta_{3}\right)-\left(1+\theta_{1}+\theta_{4}-\theta_{3}\right)\left(-1+2 \theta_{1}+\theta_{4}+\theta_{3}-\theta_{2}\right) z_{3}, \\
\mathcal{L}_{4}^{I}= & \theta_{4}\left(\theta_{1}+\theta_{4}-\theta_{3}\right)-\left(-1+\theta_{4}-\theta_{3}\right)\left(-1+2 \theta_{1}+\theta_{4}+\theta_{3}-\theta_{2}\right) z_{4} .
\end{aligned}
$$

Now we calculate the ring $\mathcal{R}$ given by the orthogonal complement of the ideal of PicardFuchs operators defined in (5.17). Using the isomorphism $\theta_{i} \mapsto J_{i}$ discussed in section 5.3 we obtain the topological basis of $H_{V}^{p, p}\left(\tilde{X}_{4}\right)$ by identification with the graded ring $\mathcal{R}^{(p)}$. Since $J_{i}$ form the trivial basis of $H^{1,1}\left(\tilde{X}_{4}\right)$ and $H^{3,3}\left(\tilde{X}_{4}\right)$ is fixed by duality to $H^{1,1}\left(\tilde{X}_{4}\right)$, the non-trivial part is the cohomology group $H_{V}^{2,2}(X)$. We calculate the ring $\mathcal{R}^{(2)}$ by choosing the basis

$$
\begin{gathered}
\mathcal{R}_{1}^{(2)}=\theta_{1}^{2}, \quad \mathcal{R}_{2}^{(2)}=\theta_{4}\left(\theta_{1}+\theta_{3}\right), \quad \mathcal{R}_{3}^{(2)}=\theta_{3}\left(\theta_{1}+\theta_{3}\right), \quad \mathcal{R}_{4}^{(2)}=\theta_{2}\left(\theta_{1}+2 \theta_{2}\right), \\
\mathcal{R}_{5}^{(2)}=\theta_{2}\left(\theta_{4}+\theta_{2}\right), \quad \mathcal{R}_{6}^{(2)}=\theta_{2}\left(\theta_{3}+\theta_{2}\right) .
\end{gathered}
$$

Then we can use the intersection $\operatorname{ring} \mathcal{C}_{0}$ to determine the topological metric $\eta^{(2)}$ of (5.14) given by

$$
\eta_{I}^{(2)}=\left(\begin{array}{cccccc}
0 & 0 & 0 & 4 & 3 & 3 \\
0 & 0 & 0 & 14 & 6 & 8 \\
0 & 0 & 0 & 18 & 10 & 10 \\
4 & 14 & 18 & 230 & 124 & 137 \\
3 & 6 & 10 & 124 & 64 & 73 \\
3 & 8 & 10 & 137 & 73 & 80
\end{array}\right)
$$

The entries are just the values of the integrals

$$
\mathcal{R}_{\alpha}^{(2)} \mathcal{R}_{\beta}^{(2)}=\left.\int_{\tilde{X}_{4}}\left(\mathcal{R}_{\alpha}^{(2)} \mathcal{R}_{\beta}^{(2)}\right)\right|_{\theta_{i} \mapsto J_{i}}
$$

where we think of it in terms of the Poincaré duals and the quartic intersections are given as the coefficients of monomials in $\mathcal{C}_{0}$. The basis $\mathcal{R}_{i}^{(3)}$ at grade $p=3$ is determined by requiring $\eta_{a b}^{(3)}=\delta_{a, h^{1,1}-b+1}$ where $h^{1,1}=4$ for the case at hand. Then the basis reads

$$
\begin{gathered}
\mathcal{R}_{1}^{(3)}=\theta_{1}\left(-\theta_{1} \theta_{4}-\theta_{2} \theta_{4}+\theta_{2} \theta_{3}\right), \quad \mathcal{R}_{2}^{(3)}=\theta_{1}\left(-\theta_{1} \theta_{4}+\theta_{1} \theta_{2}+\theta_{2} \theta_{4}-\theta_{2} \theta_{3}\right), \\
\mathcal{R}_{3}^{(3)}=\theta_{1}^{2} \theta_{4}, \quad \mathcal{R}_{4}^{(3)}=\theta_{1}\left(-2 \theta_{1} \theta_{4}-\theta_{1} \theta_{2}+\theta_{2} \theta_{3}\right) .
\end{gathered}
$$


Finally, we choose a basis of $\mathcal{R}^{(4)}$ by $\mathcal{R}^{(4)}=\left.\frac{1}{103} \mathcal{C}_{0}\right|_{J_{i} \mapsto \theta_{i}}$ such that $\eta_{a_{0}, b_{0}}^{(4)}=1$ for $\mathcal{R}^{(0)}=1$.

Phase II: Turning to the phase II of (4.22) the Stanley-Reisner ideal and the intersection numbers read

$$
\begin{aligned}
S R= & \left\{D_{1} D_{7}, D_{7} D_{9}, D_{8} D_{9}, D_{1} D_{5} D_{6}, D_{2} D_{3} D_{4}, D_{2} D_{4} D_{7}, D_{3} D_{5} D_{6} D_{8}\right\}, \\
\mathcal{C}_{0}= & J_{1}^{2} J_{4} J_{3}+2 J_{1}^{2} J_{3}^{2}+3 J_{1} J_{4} J_{3}^{2}+12 J_{1} J_{3}^{3}+9 J_{4} J_{3}^{3}+54 J_{3}^{4}+J_{1}^{2} J_{2} J_{4}+2 J_{1}^{2} J_{3} J_{2} \\
& +3 J_{1} J_{2} J_{3} J_{4}+12 J_{1} J_{3}^{2} J_{2}+9 J_{2} J_{3}^{2} J_{4}+54 J_{3}^{3} J_{2}+2 J_{1}^{2} J_{2}^{2}+3 J_{1} J_{4} J_{2}^{2}+12 J_{1} J_{3} J_{2}^{2} \\
& +9 J_{4} J_{3} J_{2}^{2}+54 J_{3}^{2} J_{2}^{2}+11 J_{1} J_{2}^{3}+9 J_{4} J_{2}^{3}+51 J_{3} J_{2}^{3}+46 J_{2}^{4}, \\
\mathcal{C}_{2}= & 24 J_{1}^{2}+36 J_{1} J_{4}+138 J_{1} J_{3}+102 J_{4} J_{3}+618 J_{3}^{2}+128 J_{1} J_{2}+102 J_{2} J_{4} \\
& +588 J_{3} J_{4}+530 J_{4}^{2}, \\
\mathcal{C}_{3}= & 660 J_{1}-540 J_{4}-3078 J_{3}-2776 J_{2},
\end{aligned}
$$

where the Kähler cone generators were given in (4.25).

The complete Picard-Fuchs system consists of four operators given by

$$
\begin{aligned}
\mathcal{L}_{1}^{I I}= & -\theta_{1}^{2}\left(\theta_{1}+\theta_{2}-\theta_{3}\right) \\
& -\left(-3+3 \theta_{1}-\theta_{3}+2 \theta_{4}\right)\left(-2+3 \theta_{1}-\theta_{3}+2 \theta_{4}\right)\left(-1+3 \theta_{1}-\theta_{3}+2 \theta_{4}\right) z_{1}, \\
\mathcal{L}_{2}^{I I}= & -\theta_{2}\left(\theta_{1}+\theta_{2}-\theta_{3}\right)\left(\theta_{2}-\theta_{3}+\theta_{4}\right)-12\left(-5+6 \theta_{2}\right)\left(-1+6 \theta_{2}\right)\left(-1+\theta_{2}-\theta_{3}\right) z_{2}, \\
\mathcal{L}_{3}^{I I}= & -\left(\theta_{2}-\theta_{3}\right)\left(-3 \theta_{1}+\theta_{3}-2 \theta_{4}\right)-\left(1+\theta_{1}+\theta_{2}-\theta_{3}\right)\left(1+\theta_{2}-\theta_{3}+\theta_{4}\right) z_{3}, \quad(\mathrm{~A} .9) \\
\mathcal{L}_{4}^{I I}= & \theta_{4}\left(\theta_{2}-\theta_{3}+\theta_{4}\right)-\left(-2+3 \theta_{1}-\theta_{3}+2 \theta_{4}\right)\left(-1+3 \theta_{1}-\theta_{3}+2 \theta_{4}\right) z_{4} .
\end{aligned}
$$

This enables us to calculate $H_{V}^{p, p}\left(\tilde{X}_{4}\right)$ as before. The basis at grade $p=2$ reads

$$
\begin{gathered}
\mathcal{R}_{1}^{(2)}=\theta_{1}^{2}, \quad \mathcal{R}_{2}^{(2)}=\theta_{2}\left(2 \theta_{1}+6 \theta_{3}\right), \quad \mathcal{R}_{3}^{(2)}=\theta_{3}\left(\theta_{1}+3 \theta_{3}\right), \quad \mathcal{R}_{4}^{(2)}=\theta_{1} \theta_{4}, \\
\mathcal{R}_{5}^{(2)}=\theta_{2}^{2}, \quad \mathcal{R}_{6}^{(2)}=\theta_{3}\left(2 \theta_{2}+2 \theta_{3}+\theta_{4}\right)+\theta_{2} \theta_{4},
\end{gathered}
$$

for which the topological metric $\eta^{(2)}$ is given by

$$
\eta^{(2)}=\left(\begin{array}{cccccc}
0 & 12 & 6 & 0 & 2 & 10 \\
12 & 2240 & 1120 & 20 & 328 & 1512 \\
6 & 1120 & 560 & 10 & 174 & 756 \\
0 & 20 & 10 & 0 & 3 & 12 \\
2 & 328 & 174 & 3 & 46 & 228 \\
10 & 1512 & 756 & 12 & 228 & 1008
\end{array}\right) .
$$


Again the basis of $H^{3,3}\left(\tilde{X}_{4}\right)$ is fixed by $\eta_{a b}^{(3)}=\delta_{a, h^{1,1}-b+1}$ to be

$$
\begin{gathered}
\mathcal{R}_{1}^{(3)}=-\frac{1}{91}\left(182 \theta_{1}^{2}+25 \theta_{2}^{2}+\theta_{1}\left(-225 \theta_{2}+85 \theta_{3}\right)\right)\left(\theta_{1}+\theta_{2}+\theta_{3}+\theta_{4}\right), \\
\mathcal{R}_{2}^{(3)}=\frac{1}{91}\left(91 \theta_{1}^{2}+10 \theta_{2}^{2}+\theta_{1}\left(\theta_{2}-57 \theta_{3}\right)\right)\left(\theta_{1}+\theta_{2}+\theta_{3}+\theta_{4}\right), \\
\mathcal{R}_{3}^{(3)}=-\theta_{1}\left(\theta_{2}-\theta_{3}\right)\left(\theta_{1}+\theta_{2}+\theta_{3}+\theta_{4}\right), \\
\mathcal{R}_{4}^{(3)}=-\frac{1}{91}\left(273 \theta_{1}^{2}+23 \theta_{2}^{2}+\theta_{1}\left(-207 \theta_{2}+60 \theta_{3}\right)\right)\left(\theta_{1}+\theta_{2}+\theta_{3}+\theta_{4}\right) .
\end{gathered}
$$

We conclude with the basis of $H^{4,4}\left(\tilde{X}_{4}\right)$ fixed by $\mathcal{R}^{(0)}=1$ as $\mathcal{R}^{(4)}=\left.\frac{1}{359} \mathcal{C}_{0}\right|_{J_{i} \mapsto \theta_{i}}$.

\section{B Further examples of fourfolds}

Here we consider a broader class of Calabi-Yau fourfolds $\left(\tilde{X}_{4}, X_{4}\right)$ that are constructed as described in section 4 by fibering Calabi-Yau threefolds $\tilde{Y}_{3}$ over $\mathbb{P}^{1}$. The threefolds we consider here are itself elliptically fibered over the two-dimensional base of the Hirzebruch surfaces $F_{n}$ for $n=0,1$,

$$
\begin{array}{ccc}
F_{n} \rightarrow & \tilde{Y}_{3} \\
& \downarrow \\
& \mathbb{P}^{1}
\end{array}
$$

Therefore, we will distinguish the constructed mirror pairs $\left(\tilde{X}_{4}, X_{4}\right)$ by the two-dimensional base $F_{n}$ we used to construct the threefold $\tilde{Y}_{3}$.

In the following we will present the toric data of the threefolds $\tilde{Y}_{3}$ and fourfolds $\tilde{X}_{4}$ including some of their topological quantities. Then we will determine the complete system of Picard-Fuchs differential operators at the large complex structure point of the mirror Calabi-Yau fourfold and calculate the holomorphic prepotential $F^{0}$. From this we extract the invariants $n_{\beta}^{g}$ which are integer in all considered cases. Furthermore we show that there exists a subsector for these invariants that reproduces the closed and open Gromov-Witten invariants of the local Calabi-Yau threefolds obtained by a suitably decompactifying the elliptic fiber of the original compact threefold. This matching allows us to determine the four-form flux $G_{4}$ for the F-theory compactification on these fourfolds such that the superpotential (3.4) admits the split (3.5) into flux and brane superpotential.

\section{B.1 Fourfold with $F_{0}$}

We start with an elliptically fibered Calabi-Yau threefold $\tilde{Y}_{3}$ with base given by the toric Fano basis of the zeroth Hirzebruch surface $F_{0}=\mathrm{P}^{1} \times \mathrm{P}^{1}$. Its polyhedron and charge 
vectors read

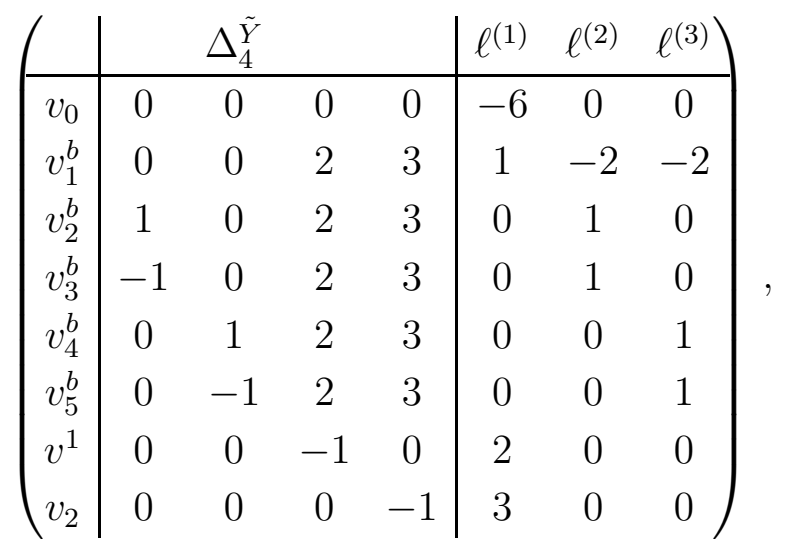

where points in the base are again labelled by a superscript ${ }^{b}$. There is one triangulation for which the Stanley-Reisner ideal in terms of the toric divisors $D_{i}=\left\{x_{i}=0\right\}$ takes the form

$$
S R=\left\{D_{2} D_{3}, D_{4} D_{5}, D_{1} D_{6} D_{7}\right\}
$$

This threefold $\tilde{Y}_{3}$ has Euler number $\chi=-480, h_{1,1}=3$ and $h_{2,1}=243$, where the three Kähler classes correspond to the elliptic fiber and the two $\mathbb{P}^{1}$ 's of the base $F_{0}$. The intersection ring for this Calabi-phase in terms of the Kähler cone generators

$$
J_{1}=D_{1}+2 D_{2}+2 D_{4}, \quad J_{2}=D_{2}, \quad J_{3}=D_{4}
$$

reads $\mathcal{C}_{0}=8 J_{1}^{3}+2 J_{1}^{2} J_{3}+2 J_{1}^{2} J_{2}+J_{1} J_{2} J_{3}$ and $\mathcal{C}_{2}=92 J_{1}+24 J_{2}+24 J_{3}$.

In the local limit $\mathcal{O}(K) \rightarrow F_{0}$ Harvey-Lawson type branes described by the brane charge vectors $\hat{\ell}^{(1)}=(-1,0,1,0,0)$ and $\hat{\ell}^{(1)}=(-1,0,0,1,0)$ were studied in [8]. To construct the Calabi-Yau fourfold $\tilde{X}_{4}$ we use the construction of section 4 with the brane vector $\hat{\ell}^{(1)}$ and expand $\Delta_{4}^{\tilde{Y}}$ to the polyhedron $\Delta_{5}^{\tilde{X}}$ and determine the Mori cone generators $\ell^{(i)}$ with $i=1, \ldots 5$ for the four different triangulations of the corresponding Calabi-Yau phases. Here we display one of the four triangulations on which we focus our following analysis: 


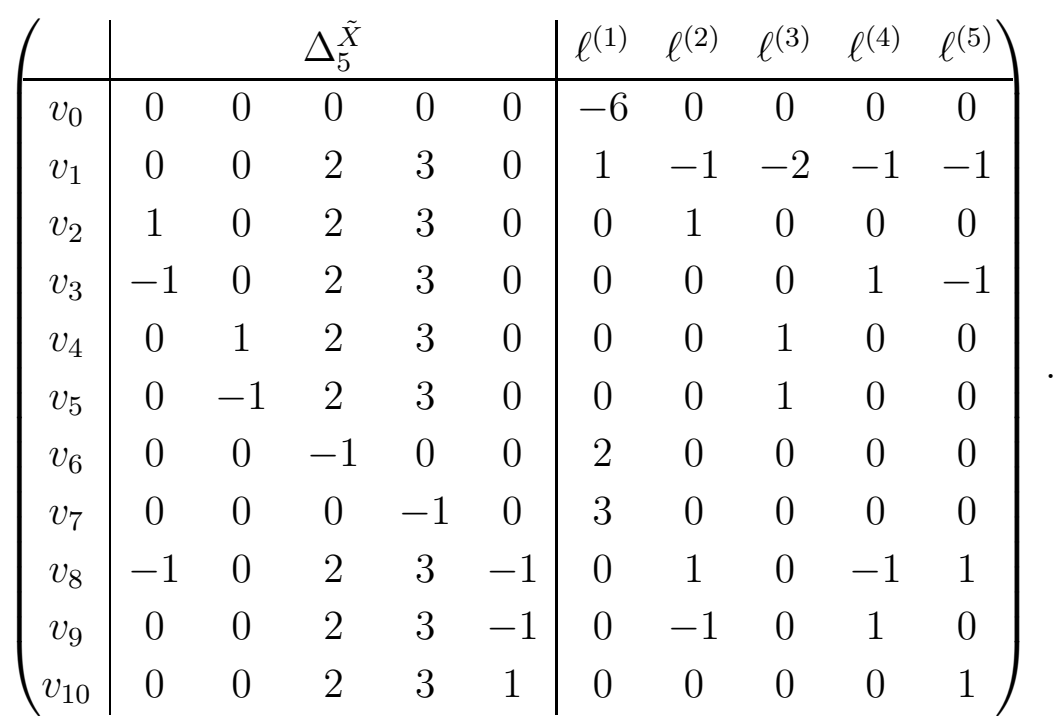

In this triangulation the Stanley-Reisner ideal takes the form

$$
S R=\left\{D_{2} D_{3}, D_{2} D_{8}, D_{3} D_{9}, D_{4} D_{5}, D_{8} D_{10}, D_{9} D_{10}, D_{1} D_{6} D_{7}\right\} .
$$

The generators of the Kähler cone of the fourfold $\tilde{X}_{4}$ in the given triangulation are $J_{1}=D_{1}+2 D_{10}+D_{2}+D_{3}+2 D_{4}, \quad J_{2}=D_{10}, \quad J_{3}=D_{4}, \quad J_{4}=D_{10}+D_{3}, \quad J_{5}=D_{2}$,

for which the intersections are determined to be

$$
\begin{aligned}
\mathcal{C}_{0}= & 42 J_{1}^{4}+8 J_{1}^{3} J_{2}+7 J_{1}^{3} J_{3}+2 J_{1}^{2} J_{2} J_{3}+12 J_{1}^{3} J_{4}+2 J_{1}^{2} J_{2} J_{4}+3 J_{1}^{2} J_{3} J_{4}+J_{1} J_{2} J_{3} J_{4} \\
& +2 J_{1}^{2} J_{4}^{2}+J_{1} J_{3} J_{4}^{2}+8 J_{1}^{3} J_{5}+2 J_{1}^{2} J_{2} J_{5}+2 J_{1}^{2} J_{3} J_{5}+J_{1} J_{2} J_{3} J_{5}+2 J_{1}^{2} J_{4} J_{5}+J_{1} J_{3} J_{4} J_{5}, \\
\mathcal{C}_{2}= & 92 J_{1} J_{2}+486 J_{1}^{2}+24 J_{2} J_{3}+82 J_{1} J_{3}+24 J_{3} J_{5}+92 J_{1} J_{5}+24 J_{2} J_{5} \\
& +24 J_{2} J_{4}+138 J_{1} J_{4}+36 J_{3} J_{4}+24 J_{4} J_{5}+24 J_{4}^{2}, \\
\mathcal{C}_{3}= & -2534 J_{1}-480 J_{2}-420 J_{3}-720 J_{4}-480 J_{5} .
\end{aligned}
$$

We calculate the core topological quantities to be

$$
\chi=15408, \quad h_{3,1}=2555, \quad h_{2,1}=0, \quad h_{1,1}=5 .
$$

Furthermore, we note that these intersections reveal the fibration structure of $\tilde{X}_{4}$. We recognize the Euler number of the threefold $\tilde{Y}_{3}$ as the coefficient of $J_{2}$ and $J_{5}$ in $\mathcal{C}_{3}$ and the fact that both $J_{2}$ and $J_{5}$ appear at most linear in $\mathcal{C}_{0}, \mathcal{C}_{2}$. This is consistent with the fact that the fiber $F$ of a fibration has intersection number 0 with itself which implies $c_{3}(F)=c_{3}\left(\tilde{X}_{4}\right)$ using the adjunction formula as well as $c_{1}(F)+c_{1}\left(N_{\tilde{X}_{4}} F\right)=c_{1}\left(N_{\tilde{X}_{4}} F\right)=0$ 
for $\tilde{X}_{4}$ Calabi-Yau. Thus we observe a fibration of $\tilde{Y}_{3}$ represented by the classes $J_{2}$ and $J_{5}$ over the base curves corresponding to $\ell^{(2)}, \ell^{(5)}$, respectively.

The Picard-Fuchs operators are determined as before and read

$$
\begin{aligned}
& \mathcal{L}_{1}=\theta_{1}\left(\theta_{1}-\theta_{2}-2 \theta_{3}-\theta_{4}-\theta_{5}\right)-12\left(-5+6 \theta_{1}\right)\left(-1+6 \theta_{1}\right) z_{1}, \\
& \mathcal{L}_{2}=\theta_{2}\left(\theta_{2}-\theta_{4}+\theta_{5}\right)-\left(-1+\theta_{2}-\theta_{4}\right)\left(-1-\theta_{1}+\theta_{2}+2 \theta_{3}+\theta_{4}+\theta_{5}\right) z_{2}, \\
& \mathcal{L}_{3}=\theta_{3}^{2}-\left(1+\theta_{1}-\theta_{2}-2 \theta_{3}-\theta_{4}-\theta_{5}\right)\left(2+\theta_{1}-\theta_{2}-2 \theta_{3}-\theta_{4}-\theta_{5}\right) z_{3}, \\
& \mathcal{L}_{4}=\left(\theta_{2}-\theta_{4}\right)\left(\theta_{4}-\theta_{5}\right)-\left(1+\theta_{2}-\theta_{4}+\theta_{5}\right)\left(-1-\theta_{1}+\theta_{2}+2 \theta_{3}+\theta_{4}+\theta_{5}\right) z_{4}, \\
& \mathcal{L}_{5}=\theta_{5}\left(\theta_{2}-\theta_{4}+\theta_{5}\right)-\left(1+\theta_{1}-\theta_{2}-2 \theta_{3}-\theta_{4}-\theta_{5}\right)\left(1+\theta_{4}-\theta_{5}\right) z_{5} .
\end{aligned}
$$

Then we can proceed with fixing the basis of $H_{V}^{(p, p)}\left(\tilde{X}_{4}\right)$ at each grade $p$ by determining the ring $\mathcal{R}$ of (5.17). We choose a basis at grade $p=2$ as

$$
\begin{aligned}
& \mathcal{R}_{1}^{(2)}=\theta_{1}\left(\theta_{1}+\theta_{5}\right), \mathcal{R}_{2}^{(2)}=\theta_{1}\left(\theta_{1}+\theta_{2}\right), \quad \mathcal{R}_{3}^{(2)}=\theta_{1}\left(2 \theta_{1}+\theta_{3}\right), \quad \mathcal{R}_{4}^{(2)}=\theta_{1}\left(\theta_{1}+\theta_{4}\right), \\
& \mathcal{R}_{5}^{(2)}=\theta_{2} \theta_{3}, \quad \mathcal{R}_{6}^{(2)}=\left(\theta_{2}+\theta_{4}\right)\left(\theta_{4}+\theta_{5}\right), \quad \mathcal{R}_{7}^{(2)}=\theta_{3} \theta_{4}, \quad \mathcal{R}_{8}^{(2)}=\theta_{3} \theta_{5} .
\end{aligned}
$$

The basis of solution dual to this basis choice is given by

$$
\begin{gathered}
\mathbb{L}_{1}^{(2)}=\frac{1}{8} l_{1}\left(l_{1}-l_{2}-2 l_{3}-l_{4}+7 l_{5}\right), \quad \mathbb{L}_{2}^{(2)}=\frac{1}{8} l_{1}\left(l_{1}+7 l_{2}-2 l_{3}-l_{4}-l_{5}\right), \\
\mathbb{L}_{3}^{(2)}=\frac{1}{4} l_{1}\left(l_{1}-l_{2}+2 l_{3}-l_{4}-l_{5}\right), \quad \mathbb{L}_{4}^{(2)}=\frac{1}{8} l_{1}\left(l_{1}-l_{2}-2 l_{3}+7 l_{4}-l_{5}\right), \quad \mathbb{L}_{5}^{(2)}=l_{2} l_{3}, \\
\mathbb{L}_{6}^{(2)}=\frac{1}{4}\left(l_{2}+l_{4}\right)\left(l_{4}+l_{5}\right), \quad \mathbb{L}_{7}^{(2)}=l_{3} l_{4}, \quad \mathbb{L}_{8}^{(2)}=l_{3} l_{5} .
\end{gathered}
$$

The topological two-point coupling between the $\mathcal{R}_{\alpha}^{(2)}$ in the chosen basis reads

$$
\eta^{(2)}=\left(\begin{array}{cccccccc}
58 & 60 & 109 & 64 & 3 & 8 & 4 & 2 \\
60 & 58 & 109 & 64 & 2 & 8 & 4 & 3 \\
109 & 109 & 196 & 118 & 4 & 20 & 6 & 4 \\
64 & 64 & 118 & 68 & 3 & 8 & 4 & 3 \\
3 & 2 & 4 & 3 & 0 & 0 & 0 & 0 \\
8 & 8 & 20 & 8 & 0 & 0 & 0 & 0 \\
4 & 4 & 6 & 4 & 0 & 0 & 0 & 0 \\
2 & 3 & 4 & 3 & 0 & 0 & 0 & 0
\end{array}\right) .
$$

The basis of $\mathcal{R}^{(3)}$ determining $H^{3,3}\left(\tilde{X}_{4}\right)$ that is fixed by Poincaré duality to the Kähler cone generators satisfying $\eta_{a b}^{(3)}=\delta_{a, h^{1,1}-b+1}$ is chosen to be

$$
\begin{gathered}
\mathcal{R}_{1}^{(3)}=\frac{1}{4}\left(9 \theta_{1} \theta_{5}-2 \theta_{1} \theta_{3}-\theta_{3}^{2}\right) \theta_{3}+\theta_{2} \theta_{3}^{2}-\theta_{1} \theta_{2} \theta_{5} \\
\mathcal{R}_{2}^{(3)}=\frac{1}{8}\left(\theta_{1} \theta_{3}+2 \theta_{3}^{2}-10 \theta_{1} \theta_{5}\right) \theta_{3}-\theta_{2} \theta_{3}^{2}-\theta_{1} \theta_{2} \theta_{5}, \quad \mathcal{R}_{3}^{(3)}=\theta_{1}\left(\frac{1}{2} \theta_{3}^{2}-\theta_{3} \theta_{5}-2 \theta_{2} \theta_{5}\right) \\
\mathcal{R}_{4}^{(3)}=\theta_{1} \theta_{2} \theta_{5}, \quad \mathcal{R}_{5}^{(3)}=\frac{1}{8} \theta_{3}\left(2 \theta_{3}^{2}-3 \theta_{1} \theta_{3}-10 \theta_{1} \theta_{5}-4 \theta_{2} \theta_{3}\right)-\theta_{1} \theta_{2} \theta_{5}
\end{gathered}
$$


We choose the basis of $H^{4,4}\left(\tilde{X}_{4}\right)$ such that the volume is normalized as $\eta_{a_{0}, b_{0}}^{(4)}=1$ for $\mathcal{R}^{(0)}=1$, i.e. $\mathcal{R}^{(4)}=\left.\frac{1}{96} \mathcal{C}_{0}\right|_{J \mapsto \theta}$.

In order to fix the integral basis of $H_{V}^{(2,2)}\left(\tilde{X}_{4}\right)$ we again match the threefold periods from the fourfold periods via (5.43). The first step is to identify the Kähler classes of $\tilde{Y}_{3}$. As discussed above $J_{5}$ represents the class of the Calabi-Yau fiber $\tilde{Y}_{3}$. The intersections of $\tilde{Y}_{3}$ are obtained from (B.8) upon the identification

$$
J_{1} \leftrightarrow J_{1}\left(\tilde{Y}_{3}\right) \quad J_{2}+J_{4}, \leftrightarrow J_{2}\left(\tilde{Y}_{3}\right), \quad J_{3} \leftrightarrow J_{3}\left(\tilde{Y}_{3}\right)
$$

With this in mind we calculate the leading logarithms $\mathbb{L}_{\alpha}\left(Y_{3}\right)$ on the threefold given by $\mathbb{L}_{1}\left(Y_{3}\right)=\frac{1}{2} X_{0}\left(2 \tilde{l}_{1}+\tilde{l}_{2}\right)\left(2 \tilde{l}_{1}+\tilde{l}_{3}\right), \quad \mathbb{L}_{2}\left(Y_{3}\right)=\frac{1}{2} X_{0} \tilde{l}_{1}\left(\tilde{l}_{1}+\tilde{l}_{3}\right), \quad \mathbb{L}_{3}\left(Y_{3}\right)=\frac{1}{2} X_{0} \tilde{l}_{1}\left(\tilde{l}_{1}+\tilde{l}_{2}\right)$.

This together with the requirement of matching the instanton numbers $19 n_{d_{1}, d_{2}, d_{3}}$ of $\tilde{Y}_{3}$ via $n_{d_{1}, d_{2}, d_{3}, d_{2}, 0}$ on $\tilde{X}_{4}$ fixes unique solutions of the Picard-Fuchs system

$\mathbb{L}_{1}^{(2)}=\frac{1}{2} X_{0}\left(2 l_{1}+l_{3}\right)\left(2 l_{1}+l_{2}+l_{4}\right), \quad \mathbb{L}_{6}^{(2)}=\frac{1}{2} X_{0} l_{1}\left(l_{1}+l_{3}\right), \quad \mathbb{L}_{8}^{(2)}=\frac{1}{2} X_{0} l_{1}\left(l_{1}+l_{2}+l_{4}\right)$,

that upon (B.16) coincide with the threefold solutions. This fixes three ring elements $\tilde{\mathcal{R}}_{\alpha}^{(2)}, \alpha=1,6,8$, by the map induced from (5.40) that we complete to a new basis

$$
\begin{gathered}
\tilde{\mathcal{R}}_{1}^{(2)}=\frac{1}{8} \theta_{1}\left(\theta_{1}-\theta_{2}-2 \theta_{3}-\theta_{4}+7 \theta_{5}\right), \quad \tilde{\mathcal{R}}_{2}^{(2)}=\frac{1}{8} \theta_{1}\left(\theta_{1}+7 \theta_{2}-2 \theta_{3}-\theta_{4}-\theta_{5}\right), \\
\tilde{\mathcal{R}}_{3}^{(2)}=\frac{1}{4} \theta_{1}\left(\theta_{1}-\theta_{2}+2 \theta_{3}-\theta_{4}-\theta_{5}\right), \quad \tilde{\mathcal{R}}_{4}^{(2)}=\frac{1}{8} \theta_{1}\left(\theta_{1}-\theta_{2}-2 \theta_{3}+7 \theta_{4}-\theta_{5}\right), \\
\tilde{\mathcal{R}}_{5}^{(2)}=\theta_{2} \theta_{3}, \quad \tilde{\mathcal{R}}_{6}^{(2)}=\frac{1}{4}\left(\theta_{2}+\theta_{4}\right)\left(\theta_{4}+\theta_{5}\right), \quad \tilde{\mathcal{R}}_{7}^{(2)}=\theta_{3} \theta_{4}, \quad \tilde{\mathcal{R}}_{8}^{(2)}=\theta_{3} \theta_{5} .(\mathrm{B}
\end{gathered}
$$

Then the integral basis elements are given by

$$
\hat{\gamma}_{1}^{(2)}=\left.\tilde{\mathcal{R}}_{1}^{(2)} \Omega\right|_{z=0}, \quad \hat{\gamma}_{6}^{(2)}=\left.\tilde{\mathcal{R}}_{6}^{(2)} \Omega\right|_{z=0}, \quad \hat{\gamma}_{8}^{(2)}=\left.\tilde{\mathcal{R}}_{8}^{(2)} \Omega\right|_{z=0},
$$

where again the new grade $p=2$ basis is obtained by replacing $l_{i} \leftrightarrow \theta_{i}$ in the dual solutions of (B.13). We conclude by presenting the leading logarithms of the periods $\Pi^{(2) \alpha}$ when integrating $\Omega$ over the duals $\gamma^{(2) \alpha}$ for $\alpha=1,6,8$. They are then as well given by $\mathbb{L}^{(2) 1}=X_{0} l_{1}\left(l_{1}+l_{5}\right), \mathbb{L}^{(2) 6}=X_{0}\left(l_{2}+l_{4}\right)\left(l_{4}+l_{5}\right)$ and $\mathbb{L}^{(2) 8}=X_{0} l_{3} l_{5}$.

Finally we determine a $\hat{\gamma}$ flux in $H_{H}^{2,2}\left(X_{4}\right)$ such that we match the disk invariants of [8] for both classes of the local geometry $\mathcal{O}(K) \rightarrow F_{0}$ with the brane class. Furthermore we reproduce the closed invariants of [56] for the two $\mathbb{P}^{1}$-classes for zero brane winding $m=0$. First we identify in the polyhedron (B.5) the vector $\ell^{(4)}$ as corresponding to the

\footnotetext{
${ }^{19}$ We note here that by just matching the threefold instantons the solution on the fourfold could not be fixed. The two free parameters could only be determined by matching the classical terms, too.
} 
brane vector. Then we expect to recover the disk invariants from the fourfold invariants $n_{0, d_{1}, d_{2}, d_{1}+m, 0}$. Then the flux $\hat{\gamma}$ deduced this way still contains a freedom of three parameters and takes the form

$$
\hat{\gamma}=\left.\left(-\mathcal{R}_{5}^{(2)}+\frac{1}{4} \mathcal{R}_{6}^{(2)}+\mathcal{R}_{7}^{(2)}+\frac{1}{2} \mathcal{R}_{8}^{(2)}\right) \Omega\right|_{z=0}
$$

where we choose the free parameters $a_{i}$ in front of $\mathcal{R}_{1}^{(2)}, \mathcal{R}_{2}^{(2)}, \mathcal{R}_{3}^{(2)}$ and $\mathcal{R}_{4}^{(2)}$ to be zero. Note that $a_{7}=1$ is fixed by the requirement of matching the disk invariants. For this parameter choice the leading logarithmic structures of the corresponding period $\int_{\gamma} \Omega$ and of the solution matching the invariants are respectively given by

$$
\mathbb{L}^{(2) \gamma}=X_{0}\left(l_{2}+l_{4}\right)\left(l_{4}+l_{5}\right), \quad \mathbb{L}_{\gamma}^{(2)}=\frac{1}{2} X_{0} l_{1}\left(4 l_{1}+3 l_{2}+2 l_{3}+l_{4}\right) .
$$

\section{B.2 Fourfold with $F_{1}$}

Here we consider an elliptically fibered Calabi-Yau threefold $\tilde{Y}_{3}$ with base twofold given by $F_{1}=\mathbb{P}(\mathcal{O} \oplus \mathcal{O}(1))$ which is the blow-up of $\mathrm{P}^{2}$ at one point. The polyhedron and charge vectors read

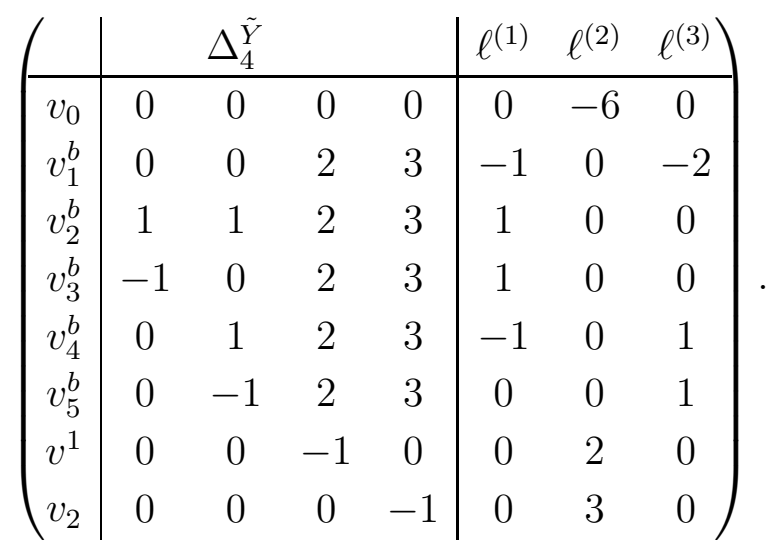

where the labels by a superscript ${ }^{b}$ again denote points in the base. There are two Calabi-Yau phases and for the triangulation given above the Stanley-Reisner ideal reads

$$
S R=\left\{D_{2} D_{3}, D_{4} D_{5}, D_{1} D_{6} D_{7}\right\}
$$

This threefold has Euler number $\chi=480, h_{1,1}=3$ and $h_{2,1}=243$, where the three Kähler classes correspond to the elliptic fiber and the two $\mathbb{P}^{1}$ 's of the base $F_{1}$. The intersection ring for this Calabi-phase in terms of the Kähler cone generators

$$
J_{1}=D_{2}, \quad J_{2}=D_{1}+3 D_{2}+2 D_{4}, \quad J_{3}=D_{2}+D_{4}
$$

reads $\mathcal{C}_{0}=2 J_{1} J_{2}^{2}+8 J_{2}^{3}+J_{1} J_{2} J_{3}+3 J_{2}^{2} J_{3}+J_{2} J_{3}^{2}$ and $\mathcal{C}_{2}=24 J_{1}+92 J_{2}+36 J_{3}$. 
For the second Calabi-Yau phase we have the following data:

$$
\begin{gathered}
\left(\begin{array}{r|rrrrrrrr}
\ell^{(1)} & -6 & 0 & 1 & 1 & -1 & 0 & 2 & 3 \\
\ell^{(2)} & 0 & -3 & 1 & 1 & 0 & 1 & 0 & 0 \\
\ell^{(3)} & 0 & 1 & -1 & -1 & 1 & 0 & 0 & 0
\end{array}\right) \\
S R=\left\{D_{1} \cdot D_{4}, D_{4} \cdot D_{5}, D_{1} \cdot D_{6} \cdot D_{7}, D_{2} \cdot D_{3} \cdot D_{5}, D_{2} \cdot D_{3} \cdot D_{6} \cdot D_{7}\right\} \\
J_{1}=D_{1}+3 D_{2}+2 D+4, \quad J_{2}=D_{2}+D_{4}, \quad J_{3}=D_{1}+3 D_{2}+3 D_{4}, \\
\mathcal{C}_{0}=8 J_{1}^{3}+3 J_{1}^{2} J_{2}+J_{1} J_{2}^{2}+9 J_{1}^{2} J_{3}+3 J_{1} J_{2} J_{3}+J_{2}^{2} J_{3}+9 J_{1} J_{3}^{2}+3 J_{2} J_{3}^{2}+9 J_{3}^{3}, \\
\mathcal{C}_{2}=92 J_{1}+36 J_{2}+102 J_{3} .
\end{gathered}
$$

Harvey-Lawson type branes were considered in [8] for the brane charge vectors $\hat{\ell}^{(1)}=$ $(-1,1,0,0,0)$ and $\hat{\ell}^{(1)}=(-1,0,0,1,0)$ for the non-compact model $\mathcal{O}(K) \rightarrow F_{1}$. The Calabi-Yau fourfold $\tilde{X}_{4}$ is constructed from the brane vector $\hat{\ell}^{(1)}$ for which there are eleven triangulations. Again we restrict our attention to one triangulation with the following data

$$
\begin{aligned}
& \left(\begin{array}{r|rrrrrrrrrrr}
\ell^{(1)} & 0 & -1 & 0 & -1 & 0 & 0 & 0 & 0 & 1 & 0 & 1 \\
\ell^{(2)} & 0 & -1 & 0 & 1 & 0 & 0 & 0 & 0 & -1 & 1 & 0 \\
\ell^{(3)} & 0 & 0 & 1 & 0 & -1 & 0 & 0 & 0 & 1 & -1 & 0 \\
\ell^{(4)} & 0 & -2 & 0 & 0 & 1 & 1 & 0 & 0 & 0 & 0 & 0 \\
\ell^{(5)} & -6 & 1 & 0 & 0 & 0 & 0 & 2 & 3 & 0 & 0 & 0
\end{array}\right), \\
& S R=\left\{D_{2} \cdot D_{3}, D_{2} \cdot D_{8}, D_{3} \cdot D_{9}, D_{4} \cdot D_{5}, D_{8} \cdot D_{10}, D_{9} \cdot D_{10}, D_{1} \cdot D_{6} \cdot D_{7}\right\} \\
& J_{1}=D_{2}, J_{2}=D_{1}+2 D_{10}+D_{2}+D_{3}+2 D_{4}, J_{3}=D_{4}, J_{4}=D_{10}, J_{5}=D_{10}+D_{3}
\end{aligned}
$$

with intersections

$$
\begin{aligned}
\mathcal{C}_{0}= & J_{1} J_{2} J_{4} J_{5}+J_{2}^{2} J_{4} J_{5}+J_{1} J_{3} J_{4} J_{5}+J_{2} J_{3} J_{4} J_{5}+J_{1} J_{4}^{2} J_{5}+J_{2} J_{4}^{2} J_{5} \\
& +2 J_{1} J_{2} J_{5}^{2}+2 J_{2}^{2} J_{5}^{2}+2 J_{1} J_{3} J_{5}^{2}+2 J_{2} J_{3} J_{5}^{2}+3 J_{1} J_{4} J_{5}^{2}+4 J_{2} J_{4} J_{5}^{2} \\
& +2 J_{3} J_{4} J_{5}^{2}+2 J_{4}^{2} J_{5}^{2}+8 J_{1} J_{5}^{3}+12 J_{2} J_{5}^{3}+8 J_{3} J_{5}^{3}+11 J_{4} J_{5}^{3}+42 J_{5}^{4}, \\
\mathcal{C}_{2}= & 24 J_{1} J_{2}+24 J_{2}^{2}+24 J_{1} J_{3}+24 J_{2} J_{3}+36 J_{1} J_{4}+48 J_{2} J_{4}+24 J_{3} J_{4}+24 J_{4}^{2} \\
& +92 J_{1} J_{5}+138 J_{2} J_{5}+92 J_{3} J_{5}+128 J_{4} J_{5}+486 J_{5}^{2}, \\
\mathcal{C}_{3}= & -480 J_{1}-270 J_{2}-480 J_{3}-660 J_{4}-2534 J_{5} .
\end{aligned}
$$

Furthermore, we determine $\chi=15408, h^{3,1}=2555, h^{2,1}=0$ and $h^{1,1}=5$.

Again the Euler number of the threefold $\tilde{Y}_{3}$ appears in $\mathcal{C}_{3}$ in front of $J_{1}$ and $J_{3}$ confirming the fibration structure. By comparing the coefficient polynomial of $J_{1}, J_{3}$ 
with the threefold intersection rings presented in appendix B.1, B.2 we infer that $J_{1}$ is precisely $\tilde{Y}_{3}=\mathcal{E} \rightarrow F_{1}$, whereas $J_{3}$ is $\tilde{Y}_{3}^{\prime}=\mathcal{E} \rightarrow F_{0}$. Since we discussed $F_{0}$ in detail before we will just concentrate on the fibration structure involving $F_{1}$.

The Picard-Fuchs operators of $X_{4}$ read as

$$
\begin{aligned}
& \mathcal{L}_{1}=\theta_{1}\left(\theta_{1}-\theta_{2}+\theta_{3}\right)-\left(-1+\theta_{1}-\theta_{2}\right)\left(-1+\theta_{1}+\theta_{2}+2 \theta_{4}-\theta_{5}\right) z_{1}, \\
& \mathcal{L}_{2}=\left(\theta_{1}-\theta_{2}\right)\left(\theta_{2}-\theta_{3}\right)-\left(1+\theta_{1}-\theta_{2}+\theta_{3}\right)\left(-1+\theta_{1}+\theta_{2}+2 \theta_{4}-\theta_{5}\right) z_{2}, \\
& \mathcal{L}_{3}=-\theta_{3}\left(\theta_{1}-\theta_{2}+\theta_{3}\right)-\left(1+\theta_{2}-\theta_{3}\right)\left(-1+\theta_{3}-\theta_{4}\right) z_{3}, \\
& \mathcal{L}_{4}=\theta_{4}\left(-\theta_{3}+\theta_{4}\right)-\left(-2+\theta_{1}+\theta_{2}+2 \theta_{4}-\theta_{5}\right)\left(-1+\theta_{1}+\theta_{2}+2 \theta_{4}-\theta_{5}\right) z_{4}, \\
& \mathcal{L}_{5}=\theta_{5}\left(-\theta_{1}-\theta_{2}-2 \theta_{4}+\theta_{5}\right)-12\left(-5+6 \theta_{5}\right)\left(-1+6 \theta_{5}\right) z_{5},
\end{aligned}
$$

from which we determine the basis of $\mathcal{R}^{(2)}$ as

$$
\begin{gathered}
\mathcal{R}_{1}^{(2)}=\left(\theta_{1}+\theta_{2}\right)\left(\theta_{2}+\theta_{3}\right), \quad \mathcal{R}_{2}^{(2)}=\theta_{1} \theta_{4}, \quad \mathcal{R}_{3}^{(2)}=\theta_{5}\left(\theta_{1}+\theta_{5}\right), \quad \mathcal{R}_{4}^{(2)}=\theta_{2} \theta_{4}, \\
\mathcal{R}_{5}^{(2)}=\theta_{5}\left(\theta_{2}+\theta_{5}\right), \quad \mathcal{R}_{6}^{(2)}=\theta_{4}\left(\theta_{3}+\theta_{4}\right), \quad \mathcal{R}_{7}^{(2)}=\theta_{3} \theta_{5}, \quad \mathcal{R}_{8}^{(2)}=\theta_{5}\left(\theta_{4}+2 \theta_{5}\right)
\end{gathered}
$$

with the two-point coupling

$$
\eta^{(2)}=\left(\begin{array}{cccccccc}
0 & 0 & 8 & 0 & 8 & 0 & 0 & 20 \\
0 & 0 & 3 & 0 & 4 & 0 & 1 & 7 \\
8 & 3 & 58 & 5 & 64 & 6 & 10 & 114 \\
0 & 0 & 5 & 0 & 5 & 0 & 1 & 9 \\
8 & 4 & 64 & 5 & 68 & 6 & 10 & 123 \\
0 & 0 & 6 & 0 & 6 & 0 & 0 & 8 \\
0 & 1 & 10 & 1 & 10 & 0 & 0 & 18 \\
20 & 7 & 114 & 9 & 123 & 8 & 18 & 214
\end{array}\right) .
$$

The dual basis of solutions reads

$$
\begin{gathered}
\mathbb{L}^{(2) 1}=\frac{1}{4}\left(l_{1}+l_{2}\right)\left(l_{2}+l_{3}\right), \quad \mathbb{L}^{(2) 2}=l_{1} l_{4}, \quad \mathbb{L}^{(2) 3}=\frac{1}{7} l_{5}\left(6 l_{1}-l_{2}-2 l_{4}+l_{5}\right), \\
\mathbb{L}^{(4) 1}=l_{2} l_{4}, \quad \mathbb{L}^{(2) 5}=\frac{1}{7} l_{5}\left(-l_{1}+6 l_{2}-2 l_{4}+l_{5}\right), \quad \mathbb{L}^{(2) 6}=\frac{1}{2} l_{4}\left(l_{3}+l_{4}\right), \quad \mathbb{L}^{(2) 7}=l_{3} l_{5}, \\
\mathbb{L}^{(2) 8}=\frac{1}{7} l_{5}\left(-2 l_{1}-2 l_{2}+3 l_{4}+2 l_{5}\right) .
\end{gathered}
$$

We determine $H^{(3,3)}\left(\tilde{X}_{4}\right)$ by duality to the canonical basis of $H^{(1,1)}\left(\tilde{X}_{4}\right)$ by the basis choice of $\mathcal{R}^{(3)}$ given as

$$
\begin{array}{ll}
\mathcal{R}_{1}^{(3)}=\theta_{1} \theta_{2} \theta_{4}, \quad \mathcal{R}_{2}^{(3)}=-2 \theta_{1} \theta_{2} \theta_{4}+\theta_{1} \theta_{2} \theta_{5}, & \mathcal{R}_{3}^{(3)}=-\theta_{1} \theta_{2} \theta_{5}+\theta_{2} \theta_{4} \theta_{5}-\theta_{3} \theta_{4} \theta_{5}, \\
\mathcal{R}_{4}^{(3)}=-\theta_{1} \theta_{2} \theta_{4}+\theta_{1} \theta_{4} \theta_{5}-\theta_{2} \theta_{4} \theta_{5}+\theta_{3} \theta_{4} \theta_{5}, & \mathcal{R}_{5}^{(3)}=-\theta_{1} \theta_{2} \theta_{4}-\theta_{1} \theta_{4} \theta_{5}+\theta_{2} \theta_{4} \theta_{5} .
\end{array}
$$


Our choice for a basis of $H^{(4,4)}\left(\tilde{X}_{4}\right)$ is given by $\mathcal{R}^{(4)}=\left.\frac{1}{106} \mathcal{C}_{0}\right|_{J_{i} \mapsto \theta_{i}}$.

Again we fix the integral basis of $H^{(2,2)}\left(\tilde{X}_{4}\right)$ by the requirement of recovering the threefold periods from the fourfold ones. We readily identify the Kähler classes of the threefold $\tilde{Y}_{3}$ among the fourfold classes as

$$
J_{2}+J_{3} \leftrightarrow J_{1}\left(\tilde{Y}_{3}\right), \quad J_{5} \leftrightarrow J_{2}\left(\tilde{Y}_{3}\right), \quad J_{4} \leftrightarrow J_{3}\left(\tilde{Y}_{3}\right)
$$

which matches the threefold intersections by identifying $J_{1} \equiv \tilde{Y}_{3}$ in the fourfold intersections (B.27). Then we calculate the classical terms of the threefold periods to be

$\mathbb{L}_{1}\left(\tilde{Y}_{3}\right)=\tilde{l}_{2}\left(\tilde{l}_{2}+\tilde{l}_{3}\right), \quad \mathbb{L}_{2}\left(\tilde{Y}_{3}\right)=\frac{1}{2}\left(2 \tilde{l}_{2}+\tilde{l}_{3}\right)\left(2 \tilde{l}_{1}+4 \tilde{l}_{2}+\tilde{l}_{3}\right), \quad \mathbb{L}_{3}\left(\tilde{Y}_{3}\right)=\frac{1}{2} l_{2}\left(2 l_{1}+3 l_{2}+2 l_{3}\right)$.

On the fourfold $X_{4}$ we determine the periods that match this leading logarithmic structure. They are given by

$$
\begin{gathered}
\mathbb{L}_{1}^{(2)}=X_{0} l_{5}\left(l_{4}+l_{5}\right), \quad \mathbb{L}_{2}^{(2)}=\frac{1}{2} X_{0}\left(l_{4}+2 l_{5}\right)\left(2\left(l_{2}+l_{3}\right)+l_{4}+4 l_{5}\right), \\
\mathbb{L}_{3}^{(2)}=\frac{1}{2} X_{0} l_{5}\left(2\left(l_{2}+l_{3}\right)+2 l_{4}+3 l_{5}\right)
\end{gathered}
$$

and immediately coincide with the threefold result using (B.32). It can be shown explicitly that the instanton series contained in the corresponding full solution matches the series on the threefold as well. The threefold invariants $n_{d_{1}, d_{2}, d_{3}}$ are obtained as $n_{0, d_{1}, d_{1}, d_{3}, d_{2}}$ from the fourfold invariants. To these solutions we associate using (5.40) ring elements $\mathcal{R}_{\alpha}^{2}, \alpha=1,3,2$, that we complete to a new basis as

$$
\begin{gathered}
\tilde{\mathcal{R}}_{1}^{(2)}=\frac{1}{4}\left(\theta_{1}+\theta_{2}\right)\left(\theta_{2}+\theta_{3}\right), \quad \tilde{\mathcal{R}}_{2}^{(2)}=\theta_{1} \theta_{4}, \quad \tilde{\mathcal{R}}_{3}^{(2)}=\frac{1}{7} \theta_{5}\left(6 \theta_{1}-\theta_{2}-2 \theta_{4}+\theta_{5}\right) \\
\tilde{\mathcal{R}}_{4}^{(2)}=\theta_{2} \theta_{4}, \quad \tilde{\mathcal{R}}_{5}^{(2)}=\frac{1}{7} \theta_{5}\left(-\theta_{1}+6 \theta_{2}-2 \theta_{4}+\theta_{5}\right), \quad \tilde{\mathcal{R}}_{6}^{(2)}=\frac{1}{2} \theta_{4}\left(\theta_{3}+\theta_{4}\right) \\
\tilde{\mathcal{R}}_{7}^{(2)}=\theta_{3} \theta_{5}, \quad \tilde{\mathcal{R}}_{8}^{(2)}=\frac{1}{7} \theta_{5}\left(-2 \theta_{1}-2 \theta_{2}+3 \theta_{4}+2 \theta_{5}\right)
\end{gathered}
$$

where we again note that the basis of dual solutions and the new ring basis coincide by $l_{i} \leftrightarrow \theta_{i}$. Then the integral basis elements read

$$
\hat{\gamma}_{1}^{(2)}=\left.\tilde{\mathcal{R}}_{1}^{(2)} \Omega\right|_{z=0}, \quad \hat{\gamma}_{2}^{(2)}=\left.\tilde{\mathcal{R}}_{2}^{(2)} \Omega\right|_{z=0}, \quad \hat{\gamma}_{3}^{(2)}=\left.\tilde{\mathcal{R}}_{3}^{(2)} \Omega\right|_{z=0}
$$

such that we obtain the full solution with the above leading parts $\mathbb{L}_{\alpha}^{(2)}$ as $\Pi_{\alpha}^{(2)}=\int \Omega \wedge \hat{\gamma}_{\alpha}$. The leading behaviour of the periods $\Pi^{(2) \alpha}$ is then given as $\mathbb{L}^{(2) 1}=X_{0}\left(l_{1}+l_{2}\right)\left(l_{2}+l_{3}\right)$, $\mathbb{L}^{(2) 2}=X_{0} l_{1} l_{4}, \mathbb{L}^{(2) 3}=X_{0} l_{5}\left(l_{1}+l_{5}\right)$, respectively

We conclude by determining the flux element $\hat{\gamma}$ in $H_{H}^{(2,2)}\left(X_{4}\right)$ that reproduces the disk invariants in the phase II of [8], where the local geometry $\mathcal{O}(K) \rightarrow F_{1}$ is considered. First we identify $\ell^{(2)}$ of the toric data in (B.27) as the vector encoding the brane physics. Therefore, we expect the fourfold invariants $n_{0, m+d_{1}, d_{1}, d_{2}, 0}$ to coincide with the 
disk invariants what can be checked in a direct calculation. The ring element yielding this result reads $\hat{\gamma}=\mathcal{R}_{4}^{(2)}$ where the free coefficients in front of the other ring elements were chosen to vanish. The leading logarithmic parts of the period $\int_{\gamma} \Omega$ and of the solution $\Pi_{\gamma}^{(2)}=\int \Omega \wedge \hat{\gamma} \equiv W_{\mathrm{D} 7}$ respectively read

$$
\mathbb{L}_{\gamma}^{(2)}=X_{0} l_{5}\left(l_{1}+l_{2}+l_{3}+l_{4}+2 l_{5}\right), \quad \mathbb{L}^{(2) \gamma}=X_{0} l_{2} l_{4} .
$$

\section{References}

[1] D. Lüst, "Intersecting brane worlds: A path to the standard model?," Class. Quant. Grav. 21, S1399 (2004) [arXiv:hep-th/0401156];

R. Blumenhagen, M. Cvetic, P. Langacker and G. Shiu, "Toward realistic intersecting D-brane models," Ann. Rev. Nucl. Part. Sci. 55 (2005) 71 [arXiv:hep-th/0502005].

[2] M. R. Douglas and S. Kachru, "Flux compactification," Rev. Mod. Phys. 79 (2007) 733 [arXiv:hep-th/0610102].

[3] R. Blumenhagen, B. Kors, D. Lust and S. Stieberger, "Four-dimensional String Compactifications with D-Branes, Orientifolds and Fluxes," Phys. Rept. 445, 1 (2007) [arXiv:hep-th/0610327].

[4] F. Denef, "Les Houches Lectures on Constructing String Vacua," arXiv:0803.1194 [hep-th].

[5] E. Witten, "Branes and the dynamics of QCD," Nucl. Phys. B 507 (1997) 658 [arXiv:hep-th/9706109].

[6] E. Witten, "Chern-Simons Gauge Theory As A String Theory," Prog. Math. 133 (1995) 637 [arXiv:hep-th/9207094].

[7] M. Aganagic and C. Vafa, "Mirror symmetry, D-branes and counting holomorphic discs," arXiv:hep-th/0012041.

[8] M. Aganagic, A. Klemm and C. Vafa, "Disk instantons, mirror symmetry and the duality web," Z. Naturforsch. A 57, 1 (2002) [arXiv:hep-th/0105045].

[9] W. Lerche, P. Mayr and N. Warner, "N=1 special geometry, mixed Hodge variations and toric geometry," arXiv:hep-th/0208039;

W. Lerche, P. Mayr and N. Warner, "Holomorphic N = 1 special geometry of openclosed type II strings," arXiv:hep-th/0207259. 
[10] J. Walcher, "Calculations for Mirror Symmetry with D-branes," arXiv:0904.4905 [hep-th];

D. Krefl and J. Walcher, "Real Mirror Symmetry for One-parameter Hypersurfaces," JHEP 0809, 031 (2008) [arXiv:0805.0792 [hep-th]];

D. R. Morrison and J. Walcher, "D-branes and Normal Functions," arXiv:0709.4028 [hep-th];

J. Walcher, "Opening mirror symmetry on the quintic," Commun. Math. Phys. 276, 671 (2007) [arXiv:hep-th/0605162].

[11] J. Knapp and E. Scheidegger, "Matrix Factorizations, Massey Products and F-Terms for Two-Parameter Calabi-Yau Hypersurfaces," arXiv:0812.2429 [hep-th];

J. Knapp and E. Scheidegger, "Towards Open String Mirror Symmetry for OneParameter Calabi-Yau Hypersurfaces," arXiv:0805.1013 [hep-th].

[12] H. Jockers and M. Soroush, "Relative periods and open-string integer invariants for a compact Calabi-Yau hypersurface," arXiv:0904.4674 [hep-th];

H. Jockers and M. Soroush, "Effective superpotentials for compact D5-brane CalabiYau geometries," arXiv:0808.0761 [hep-th].

[13] W. Lerche and P. Mayr, "On N = 1 mirror symmetry for open type II strings," arXiv:hep-th/0111113;

P. Mayr, "N = 1 mirror symmetry and open/closed string duality," Adv. Theor. Math. Phys. 5 (2002) 213 [arXiv:hep-th/0108229].

[14] M. Alim, M. Hecht, P. Mayr and A. Mertens, "Mirror Symmetry for Toric Branes on Compact Hypersurfaces," arXiv:0901.2937 [hep-th].

[15] T. W. Grimm, T. W. Ha, A. Klemm and D. Klevers, "The D5-brane effective action and superpotential in N=1 compactifications," Nucl. Phys. B 816 (2009) 139 [arXiv:0811.2996 [hep-th]].

[16] M. Baumgartl, I. Brunner and M. R. Gaberdiel, "D-brane superpotentials and RG flows on the quintic," JHEP 0707 (2007) 061 [arXiv:0704.2666 [hep-th]].

[17] S. Gukov, C. Vafa and E. Witten, "CFT's from Calabi-Yau four-folds," Nucl. Phys. B 584 (2000) 69 [Erratum-ibid. B 608 (2001) 477] [arXiv:hep-th/9906070].

[18] D. Lust, P. Mayr, S. Reffert and S. Stieberger, "F-theory flux, destabilization of orientifolds and soft terms on D7-branes," Nucl. Phys. B 732 (2006) 243 [arXiv:hepth/0501139]. 
[19] S. B. Giddings, S. Kachru and J. Polchinski, "Hierarchies from fluxes in string compactifications," Phys. Rev. D 66, 106006 (2002) [arXiv:hep-th/0105097].

[20] R. P. Thomas, "Moment maps, monodromy and mirror manifolds," arXiv:math/0104196.

[21] S. Hosono, A. Klemm, S. Theisen and S. T. Yau, "Mirror Symmetry, Mirror Map And Applications To Calabi-Yau Hypersurfaces," Commun. Math. Phys. 167, 301 (1995) [arXiv:hep-th/9308122].

[22] P. Candelas, X. C. De La Ossa, P. S. Green and L. Parkes, "A pair of Calabi-Yau manifolds as an exactly soluble superconformal theory," Nucl. Phys. B 359 (1991) 21.

[23] K. Hori and C. Vafa, "Mirror symmetry," arXiv:hep-th/0002222.

[24] V. V. Batyrev, "Dual polyhedra and mirror symmetry for Calabi-Yau hypersurfaces in toric varieties," J. Alg. Geom. 3, 493 (1994).

[25] A. Klemm, B. Lian, S. S. Roan and S. T. Yau, "Calabi-Yau fourfolds for M- and F-theory compactifications," Nucl. Phys. B 518, 515 (1998) [arXiv:hep-th/9701023].

[26] E. Witten, "Phases of $\mathrm{N}=2$ theories in two dimensions," Nucl. Phys. B 403 (1993) 159 [arXiv:hep-th/9301042].

[27] N. C. Leung and C. Vafa, "Branes and toric geometry," Adv. Theor. Math. Phys. 2 (1998) 91 [arXiv:hep-th/9711013].

[28] A. Strominger, S. T. Yau and E. Zaslow, "Mirror symmetry is T-duality," Nucl. Phys. B 479 (1996) 243 [arXiv:hep-th/9606040].

[29] C. Vafa, "Evidence for F-Theory," Nucl. Phys. B 469, 403 (1996) [arXiv:hepth/9602022].

[30] M. Bershadsky, K. A. Intriligator, S. Kachru, D. R. Morrison, V. Sadov and C. Vafa, "Geometric singularities and enhanced gauge symmetries," Nucl. Phys. B 481, 215 (1996) [arXiv:hep-th/9605200].

[31] A. Sen, "Orientifold limit of F-theory vacua," Phys. Rev. D 55 (1997) 7345 [arXiv:hep-th/9702165].

[32] P. Candelas and A. Font, "Duality between the webs of heterotic and type II vacua," Nucl. Phys. B 511, 295 (1998) [arXiv:hep-th/9603170]. 
[33] P. Candelas, E. Perevalov and G. Rajesh, "Toric geometry and enhanced gauge symmetry of F-theory/heterotic vacua," Nucl. Phys. B 507, 445 (1997) [arXiv:hepth/9704097].

[34] E. Witten, "On flux quantization in M-theory and the effective action," J. Geom. Phys. 22 (1997) 1 [arXiv:hep-th/9609122].

[35] M. Haack and J. Louis, "M-theory compactified on Calabi-Yau fourfolds with background flux," Phys. Lett. B 507, 296 (2001) [arXiv:hep-th/0103068].

[36] T. W. Grimm, T. W. Ha, A. Klemm and D. Klevers, "Five-Brane Superpotentials and Heterotic/F-theory Duality," arXiv:0912.3250 [hep-th].

[37] B. R. Greene, D. R. Morrison and M. R. Plesser, "Mirror manifolds in higher dimension," Commun. Math. Phys. 173 (1995) 559 [arXiv:hep-th/9402119].

[38] P. Mayr, "Mirror symmetry, $\mathrm{N}=1$ superpotentials and tensionless strings on CalabiYau four-folds," Nucl. Phys. B 494, 489 (1997) [arXiv:hep-th/9610162].

[39] A. C. Avram, M. Kreuzer, M. Mandelberg and H. Skarke, "Searching for K3 fibrations," Nucl. Phys. B 494, 567 (1997) [arXiv:hep-th/9610154].

[40] A. Sen, "F-theory and Orientifolds," Nucl. Phys. B 475, 562 (1996) [arXiv:hepth/9605150].

[41] B. Andreas, "N = 1 heterotic/F-theory duality," Fortsch. Phys. 47 (1999) 587 [arXiv:hep-th/9808159].

[42] D. R. Morrison and C. Vafa, "Compactifications of F-Theory on Calabi-Yau Threefolds - I," Nucl. Phys. B 473 (1996) 74 [arXiv:hep-th/9602114];

D. R. Morrison and C. Vafa, "Compactifications of F-Theory on Calabi-Yau Threefolds - II," Nucl. Phys. B 476 (1996) 437 [arXiv:hep-th/9603161].

[43] M. Bershadsky, A. Johansen, T. Pantev and V. Sadov, "On four-dimensional compactifications of F-theory," Nucl. Phys. B 505 (1997) 165 [arXiv:hep-th/9701165].

[44] P. Berglund and P. Mayr, "Heterotic string/F-theory duality from mirror symmetry," Adv. Theor. Math. Phys. 2, 1307 (1999) [arXiv:hep-th/9811217];

P. Berglund and P. Mayr, "Stability of vector bundles from F-theory," JHEP 9912 , 009 (1999) [arXiv:hep-th/9904114].

[45] R. Friedman, J. Morgan and E. Witten, "Vector bundles and F theory," Commun. Math. Phys. 187 (1997) 679 [arXiv:hep-th/9701162]. 
[46] S. Katz, P. Mayr and C. Vafa, "Mirror symmetry and exact solution of 4D N = 2 gauge theories. I," Adv. Theor. Math. Phys. 1, 53 (1998) [arXiv:hep-th/9706110].

[47] G. Curio and R. Y. Donagi, "Moduli in $\mathrm{N}=1$ heterotic/F-theory duality," Nucl. Phys. B 518 (1998) 603 [arXiv:hep-th/9801057].

[48] P. Candelas, A. Font, S. H. Katz and D. R. Morrison, "Mirror symmetry for two parameter models. 2," Nucl. Phys. B 429, 626 (1994) [arXiv:hep-th/9403187].

[49] E. Witten, "Mirror manifolds and topological field theory," arXiv:hep-th/9112056.

[50] K. Hori et al., "Mirror symmetry," Providence, USA: AMS (2003) 929 p

[51] S. Hosono, A. Klemm, S. Theisen and S. T. Yau, "Mirror symmetry, mirror map and applications to complete intersection Calabi-Yau spaces," Nucl. Phys. B 433 (1995) 501 [arXiv:hep-th/9406055].

[52] A. Libgober, "Chern Classes and the periods of mirrors", arXiv:math/9803119.

[53] R. Minasian and G. W. Moore, "K-theory and Ramond-Ramond charge," JHEP 9711, 002 (1997) [arXiv:hep-th/9710230].

[54] Y. K. Cheung and Z. Yin, "Anomalies, branes, and currents," Nucl. Phys. B 517, 69 (1998) [arXiv:hep-th/9710206].

[55] A. Klemm and R. Pandharipande, "Enumerative geometry of Calabi-Yau 4-folds," Commun. Math. Phys. 281, 621 (2008) [arXiv:math/0702189].

[56] B. Haghighat, A. Klemm and M. Rauch, "Integrability of the holomorphic anomaly equations," JHEP 0810 (2008) 097 [arXiv:0809.1674 [hep-th]].

[57] P. S. Aspinwall and D. R. Morrison, "Topological field theory and rational curves," Commun. Math. Phys. 151, 245 (1993) [arXiv:hep-th/9110048].

[58] R. Gopakumar and C. Vafa, "M-theory and topological strings. II," arXiv:hepth/9812127.

[59] C.-C. M. Liu, Moduli of J-holomorphic curves with Lagrangian Boundary Conditions and open Gromov-Witten Invariants for an $S^{1}$-Equivariant Pair, arXiv:math/0210257.

[60] H. Ooguri and C. Vafa, "Knot invariants and topological strings," Nucl. Phys. B 577, 419 (2000) [arXiv:hep-th/9912123]. 
[61] C. Beasley, J. J. Heckman and C. Vafa, "GUTs and Exceptional Branes in F-theory - I," JHEP 0901 (2009) 058 [arXiv:0802.3391 [hep-th]];

C. Beasley, J. J. Heckman and C. Vafa, "GUTs and Exceptional Branes in F-theory - II: Experimental Predictions," JHEP 0901 (2009) 059 [arXiv:0806.0102 [hep-th]].

[62] R. Donagi and M. Wijnholt, "Model Building with F-Theory," arXiv:0802.2969 [hepth].

[63] R. Blumenhagen, T. W. Grimm, B. Jurke and T. Weigand, "Global F-theory GUTs," arXiv:0908.1784 [hep-th].

[64] J. Marsano, N. Saulina and S. Schafer-Nameki, "F-theory Compactifications for Supersymmetric GUTs," JHEP 0908, 030 (2009) [arXiv:0904.3932 [hep-th]]. 